\title{
Molecular evolution of primates - featuring mobile elements
}

\author{
Dissertation \\ zur Erlangung des Doktorgrades \\ der Mathematisch-Naturwissenschaftlichen Fakultäten \\ der Georg-August-Universität zu Göttingen
}

vorgelegt von

Dipl. Biol.

Martin Osterholz

aus

Brunsbüttel

Göttingen, September 2008 
Referent: Prof. Dr. Peter Kappeler Korreferent: Prof. Dr. Ernst Wimmer Tag der mündlichen Prüfung: 22.10.2008 



\section{Contents}

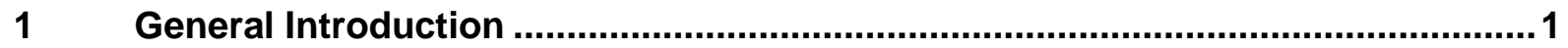

1.1 Who with Whom - An Introduction into Primate Systematics............................ 1

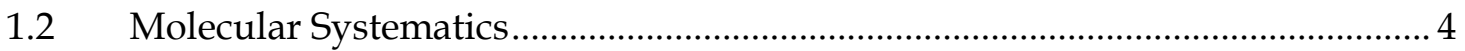

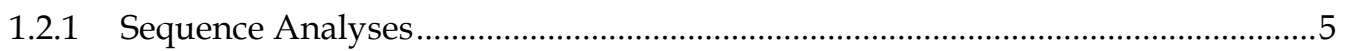

1.2.2 Mobile elements - temporal landmarks in evolution ...........................................6

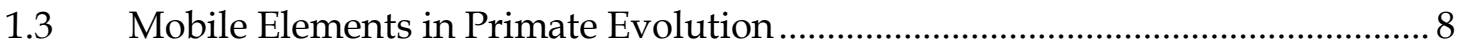

2 Retropositional events consolidate the branching order among New World

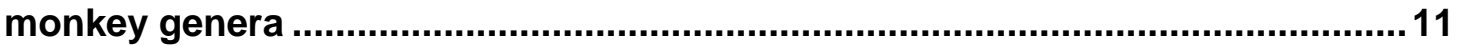

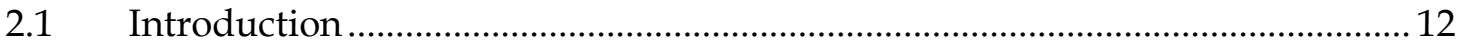

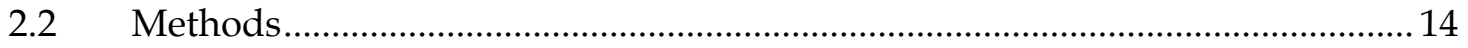

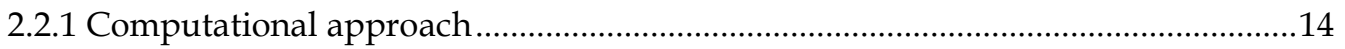

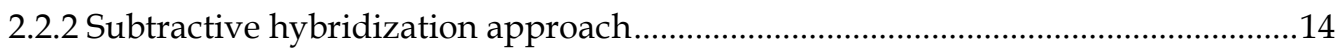

2.2.3 Testing the presence/absence of insertions in New World monkeys .....................15

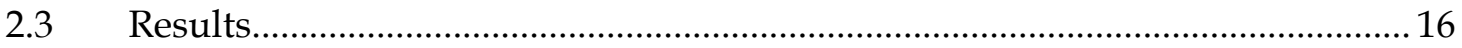

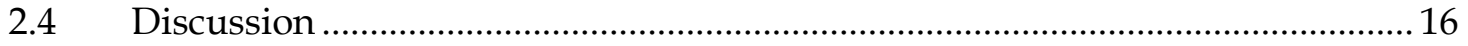

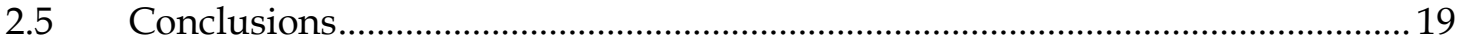

3 A PCR-based marker to simply identify Saimiri sciureus and S. boliviensis

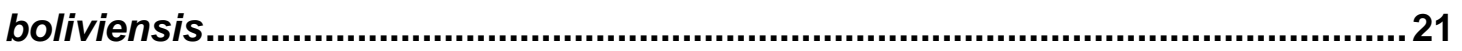

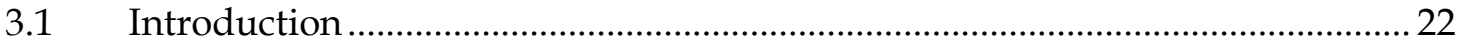

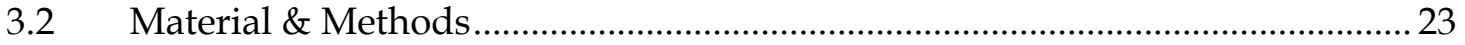

3.2.1 Database approach …………………………………..............................................23

3.2.2 Laboratory methods ……………………………………………………………...23

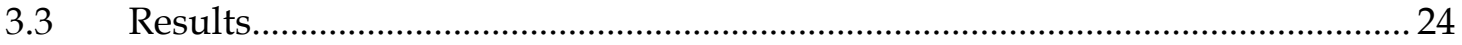

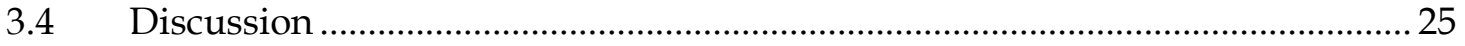

4 Phylogenetic position of the langur genera Semnopithecus and Trachypithecus among Asian colobines, and affiliations of species groups .............................. 27

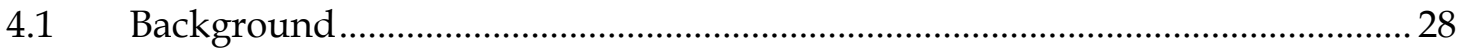

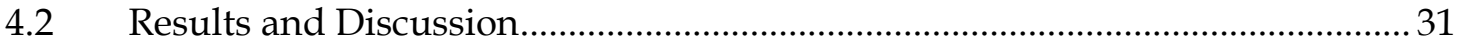

4.2.1 Genus level phylogeny.................................................................................................

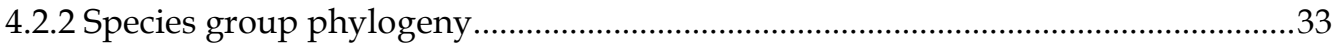




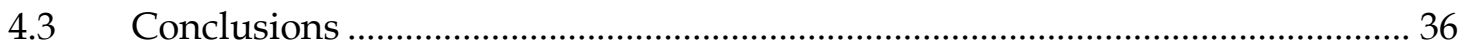

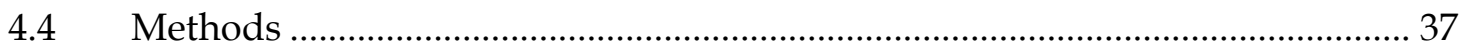

4.4.1 Sample collection, DNA extraction and preventing contaminations .......................37

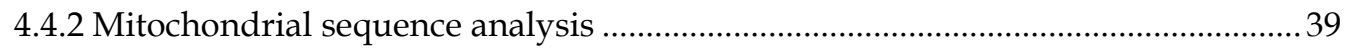

4.4.3 Y chromosomal sequence analysis ........................................................................ 41

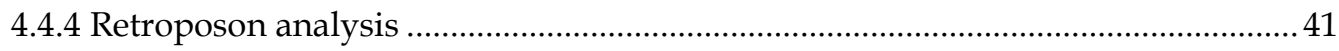

5 Mitochondrial versus nuclear DNA: The complex evolutionary history of

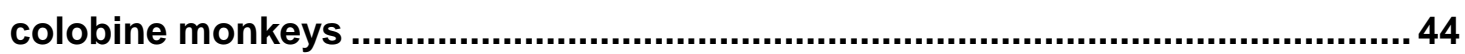

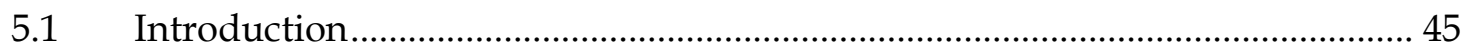

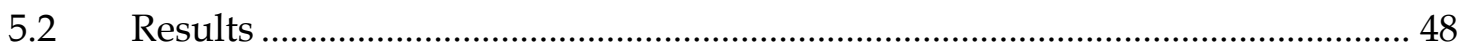

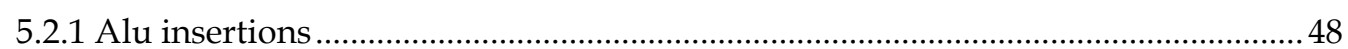

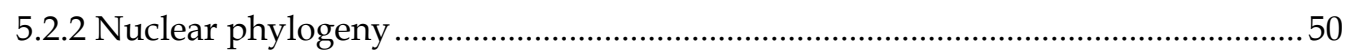

5.2.3 Mitochondrial phylogeny ........................................................................................ 51

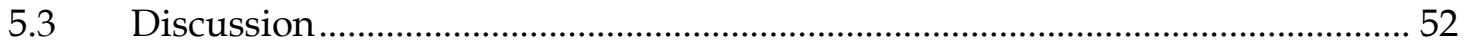

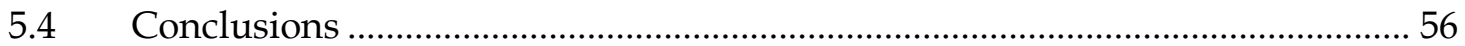

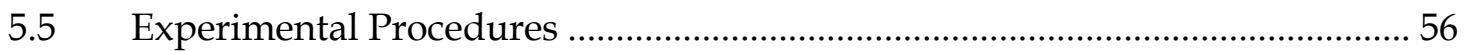

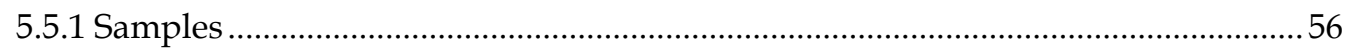

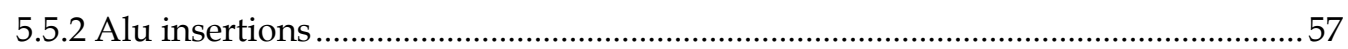

5.5.3 PCR and sequencing of nuclear loci...................................................................... 57

5.5.4 Phylogenetic reconstructions ................................................................................ 58

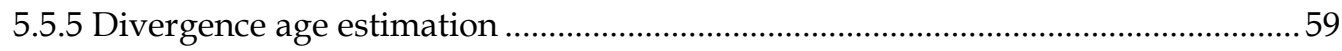

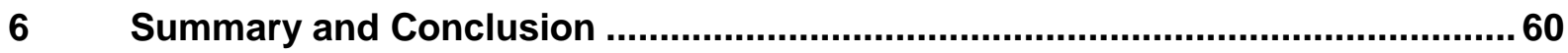

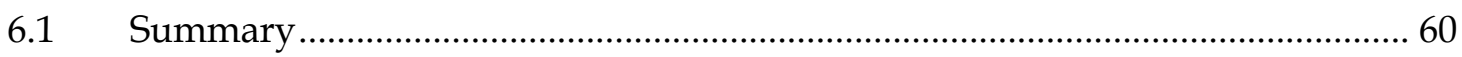

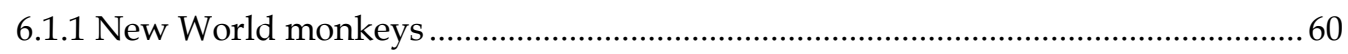

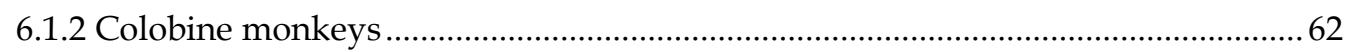

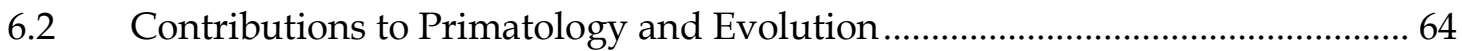

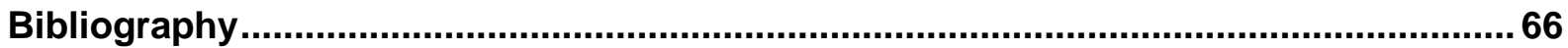

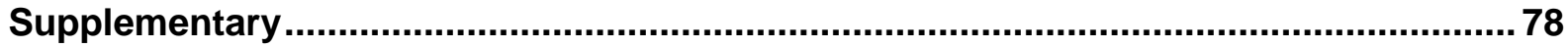

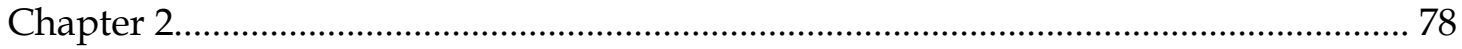

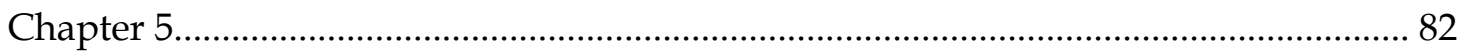





\section{General Introduction}

\subsection{Who with Whom - An Introduction into Primate Systematics}

The order Primates is one of the most diverse mammalian orders. With the exception of the genus Homo, primates are mainly distributed in tropical and subtropical regions of Africa, Asia and Central and South America. The order was first recognized in the $18^{\text {th }}$ century by Linnaeus and was divided into four different groups with 35 species. Today, the number of species varies from 230 (Rowe, 1996) to more than 350 (Groves, 2001; Geissmann, 2003) and is solely exceeded in number by Rodentia and Chiroptera. However, the identification of new species is still in progress. In the last few years, several new subspecies, species and even genera were described (Jones et al., 2005; Kappeler et al., 2005; Sinha et al., 2005; Andriaholinirina et al., 2006; Davenport et al., 2006; Wallace et al., 2006; Boubli et al., 2008; Roos et al., 2008). This phenomenon is not only based on the application of molecular methods, which allow to determine genetic diversity, but also on the increasing numbers of behavioral and ecological studies and the discovery of taxa in former unexplored areas.

The definition of the order Primates and its classification in its own order beyond other mammals is a complex issue, at least based on morphological characteristics. Mivart (1873) removed Chiroptera and Dermoptera from Primates and proposed different significant features leading to their uniqueness among other mammals. Examples are the retention of the clavicle in the pectoral girdle, a reduced number and three different types of teeth, a caecum, a penis pendulous and testes scrotal. Opposing thumbs combined with fingernails rather than claws afford gripping branches. However, not all these characteristics are synapomorphic and can also be found to some degree in non-primate orders (Geissmann, 2003). For a more adequat characterization of primates Martin (1990) provides further features. Primates have a trend towards reduced snout and olfactory senses. In contrast, the visual system is complex with high acuity and color vision and the brain is large compared to body size. Most of these characteristics can not be detected in fossils and therefore it is difficult to determine affiliations of fossil records to extant primates or to the origin of all primates respectively. It is actually unlikely to determine the origin and age of the order, because only few fossil records are available. It is assumed that only $7 \%$ of all extinct primates are known from fossils (Tavaré et al., 2002). However, based on these few records (e.g. Purgatorius, North America), the most recent common ancestor most likely lived about 65 million years ago (mya) (Shoshani et al., 1996). However, by combining fossil records with statistical analyses, the origin of primates was recently dated back to 81.5 mya (Tavaré et al., 2002). 
Genetic analyses form another possibility to detect primate-specific characteristics. Based on these studies, it is now widely accepted that the order Primates is a monophyletic group tightly clustering together with Scandentia and Dermoptera (Murphy et al., 2001a; Murphy et al., 2001b; Schmitz et al., 2005; Kriegs et al., 2006). However, mitochondrial sequence analyses indicated that dermopterans form a clade with anthropoid primates to the exclusion of other primates (Murphy et al., 2001a; Arnason et al., 2002; Schmitz et al., 2002a), leading to the assumption that primates are paraphyletic. Other studies using mobile elements, which insert into the genome and serve as molecular synapomorphic traits, clearly confirmed the monophyly of the order to the exclusion of dermopterans and other mammalian orders (Schmitz et al., 2002a; Schmitz and Zischler, 2003). Moreover, it was shown that Alu elements are a special type of short interspersed elements (SINEs), which are only present in primates, representing therefore another hint for a common origin of primates (Schmitz et al., 2002a).

Primates were originally divided into Prosimii (lemurs, lorises and tarsiers) and Anthropoidea (monkeys and apes), mainly based on morphological similarities between tarsier, lemurs and lorises. The affiliation of the tarsiers was one of the most basic questions in primate phylogeny. Even sequence data were not able to resolve this problem with significance (Andrews et al., 1998; Goodman et al., 1998; Schmitz et al., 2002b). Finally, SINE insertions provided strong molecular evidence for a sister relationship of tarsier to monkeys and apes, leading to the division into Strepsirrhini and Haplorrhini (Kuryshev et al., 2001; Schmitz et al., 2001; Schmitz et al., 2005). Morphologically, Strepsirrhini differ from Haplorhini in having a post-orbital bar, a moist nose, a tapetum lucidum (except some Lemuriformes taxa) and a dental comb. Hence, the current consensus view distinguishes two suborders, the "wet-nosed" (Strepsirrhini) and "dry-nosed" primates (Haplorrhini) (Groves, 2001; Geissmann, 2003), which diverged from each other 63-78 mya - depending on the applied calibration points (Goodman et al., 1998; Steiper and Young, 2006).

Strepsirrhini are a remarkable diverse group, comprising actually about $21 \%$ of all primate genera. They are traditionally divided into two infraorders, the Malagasy Lemuriformes (lemurs) and the Lorisiformes (lorises and galagos), which occur in Africa and Asia (Geissmann, 2003). They diverged from each other about 50-57 mya (Goodman et al., 1998; Roos et al., 2004; Steiper and Young, 2006). However, Groves (2001) separates the aye-aye from the Lemuriformes (Daubentonia madagascariensis) in its own infraorder Chiromyiformes, which seems to be appropriate due to several morphological autapomorphies, chromosomal and genetic studies and the early separation from the other Lemuriformes (Yoder et al., 1996; Groves, 2001; Roos et al., 2004; Warter et al., 2005; Mittermeier et al., 2006; Horvath et al., 2008). The respective monophyly of Lorisiformes and Lemuriformes is confirmed by several morphological features (Groves, 2001; Mittermeier et al., 2006) and molecular analyses (Yoder et al., 1996; Roos et al., 2004; Warter et al., 2005; Horvath et al., 2008). Besides the ayeaye, lemurs are further divided into four different families (Lemuridae, Indriidae, Lepilemuridae and Cheirogaleidae), whereas the lorises are classified into two families (Galagonidae and Lorisidae) (Groves, 2001). 
Haplorrhini are divided into Tarsiiformes (tarsiers) and Anthropoidea, which include Platyrrhini (New World monkeys) and Catarrhini (Old World monkeys, apes and humans). The divergence for Tarsiiformes occurred relatively early about 58 mya (Goodman et al., 1998).

Platyrrhini are a tremendously diverse group distributed in South and Central America and diverged from the Catarrhini about 40-43 mya (Goodman et al., 1998; Steiper and Young, 2006). This diversity is manifested in the presence of up to thirteen sympatric species (Fleagle, 1988). There is no consensus about how many families should be recognized, although most recent studies arrange them into three families (Cebidae (e.g. marmosets, tamarins and squirrel monkeys), Atelidae (howler, spider and woolly monkeys) and Pitheciidae (titis, sakis and uakaris), Opazo et al., 2006; Goodman et al., 1998). Alternative classifications with two (Napier and Napier, 1967), four (Groves, 2001) or even five families (Geissmann, 2003) were also proposed. The branching order of Cebidae, Atelidae and Pitheciidae is still an intensely discussed issue, because morphological as well as molecular studies produced incongruent results (e.g. Kay, 1990; Opazo et al., 2006; Ray et al. 2005; Rosenberger, 1981; Schneider et al., 1996). It is unclear whether Pitheciidae or Atelidae form the most basal family. Moreover, it is not evident whether Cebidae, including the subfamily Callithrichinae, really form a monophyletic unity.

Within the Catarrhini two different superfamilies can be distinguished, with tail (Cercopithecoidea) and without tail (Hominoidea). They diverged from each other about 23-30 mya (Goodman et al., 1998; Raaum et al., 2005; Steiper and Young, 2006). Old World monkeys or Cercopithecidae, the single family within Cercopithecoidea, are further divided into two different subfamilies: Colobinae (leaf-eating monkeys) and Cercopithecinae (cheek-pouched monkeys) (Groves, 2001).

Both subfamilies diverged from each other about 14-16 mya (Goodman et al., 1998; Raaum et al., 2005), are highly diverse and are distributed in Africa and Asia, although Cercopithecinae are more diverse in Africa and Colobinae more in Asia. Both have different adaptation to their diet, leading to different anatomical features. Colobine monkeys have a complex multichambered stomach particularly to digest leaves and fruits. In contrast, Cercopithecinae possess cheek pouches and are omnivorous (Strasser and Delson, 1987). Colobine monkeys can further be divided into an African clade (tribe Colobini) and an Asian clade (tribe Presbytini) (Groves, 2001). Latter include a langur (e.g. hanuman langur and delacour langur) and an odd-nosed monkey group (e.g. proboscis monkey and snub-nosed monkey). Especially in the evolutionary history of colobine monkeys are a lot of debated and unanswered questions, e.g. the respective monophyly of Asian and African colobines. Unfortunately, only few molecular and morphological studies provide data for them, since material is limited due to their partial endangered status and difficulties in captive breeding. Cercopithecinae are divided into the two tribes Cercopithecini (vervet monkeys and guenons) and Papionini (macaques, baboons, mandrills, gelada and mangabeys), mainly based on chromosomal (42 chromosomes in Papioni, variable number in Cercopithecini) and behavioral data (Groves, 2001). A long standing issue 
in papionine phylogeny was the paraphyly of the mangabeys (Disotell, 1994). In cercopithecine phylogeny molecular data provided evidence for an arboreal-terrestrial split (Xing et al., 2007).

The superfamily Hominoidea is divided into two families: lesser apes or gibbons (Hylobatidae) and great apes and humans (Hominidae) (Groves, 2001). Gibbons are divided into four different genera (Roos and Geissmann, 2001) and form the first split about 18 mya (Goodman et al., 1998) and are only distributed in Asia. They are well known for their characteristic brachiation movement and singing duets. Traditionally, great apes and human were divided into two families, Pongidae including all great apes and Hominidae, which comprises only human (Napier and Napier, 1967). However, molecular and also morphological studies led to a revision of this classification, including now gorilla and chimpanzee in the Homininae (Goodman et al., 1990; Groves, 2001; Salem et al., 2003) and the orang-utan in Ponginae, while latter diverged from the Homininae about 14-18 mya (Goodman et al., 1998; Steiper and Young, 2006). Among the African great apes, the gorillas were separated from the lineage leading to chimpanzee and human about 7-9 mya (Goodman et al., 1998; Steiper and Young, 2006). The latter two diverged from each other about 6 mya (Goodman et al., 1998; Raaum et al., 2005; Steiper and Young, 2006).

\subsection{Molecular Systematics}

Systematics is an essential biological discipline dealing with the study of biodiversity and evolutionary relationships of organisms in past and present. In 1758, Linnaeus Systema Naturae forms the first fundament for a hierarchic system of life. Wallace (1858) and Darwin (1859) independently expanded this fundament with the theory of natural selection as the reason for speciation. Based on the cladistic concept of Hennig (1950), particularly derived traits (apomorphies), instead of ancestral traits (plesiomorphies), are used to determine the phylogenetic relationships of organisms and to classify them. Systematics is not only the basis for all studies of organism, but also relevant for conservation interests when for instance evaluating the preservation of genetic diversity, i.e. determining evolutionary siginificant units (Ryder, 1986). Molecular systematics started in the middle of the $20^{\text {th }}$ century to expand the knowledge on the evolutionary relationships of organisms, which was established by classical disciplines in terms of morphology, paleontology or behavioral studies. First attempts were done by studying structures of proteins or chromosomes. These techniques were partly replaced and expanded by DNA-DNA hybridization (Britten and Kohne, 1968), although this method showed only distances between two organisms instead of distinct traits. Another method represented RFLP (restriction fragment length polymorphism), with which it was possible to detect variations in the DNA sequence via restriction enzymes. After DNA sequencing became an important tool in the 70s and PCR in the 80s, the analysis of multiple sequences displaced many of the former methods and was further improved by numerous statistical techniques. However, all these different methods are limited by homoplasy, a character similarity, which is not derived from a common 
ancestor leading to misgrouping of taxa. Future research in systematics will focus not only on marker genes, but also on comparative whole genome analyses, as DNA sequencing has been improved in efficiency (highthroughput DNA sequencing). But evolutionary analyses based on sequence information from complete genome can also be hampered by homoplasies, whereas rare genomic changes (e.g. random insertions and deletions (indels) or retrotransposons) form more reliable tools. Homoplasies were reported however for indels as well (de Jong et al., 2003), in contrast to retrotransposons.

In the 90s the application of mobile elements, in particular retroposons, as a phylogenetic marker system was proposed with almost homoplasy-free character (Ryan and Dugaiczyk, 1989; Okada, 1991; Murata et al., 1993). Shedlock and Okada (2000) even classified these elements as nearly perfect genetic apomorphies in terms of Hennig (1950). Since that time, retroposons have helped to elucidate several controversial phylogenetic relationships in mammalian, avian, reptile and fish phylogeny (Shimamura et al., 1997; Takahashi et al., 1998, 2001; Roos et al., 2004; Schmitz et al., 2005; Xing et al., 2005; Kriegs et al., 2006; Piskurek et al., 2006; Kriegs et al., 2007; Matveev et al., 2007; Xing et al., 2007).

\subsubsection{Sequence Analyses}

Since the establishment of PCR and sequencing methods in the 80s, the analysis of nucleotide sequences of marker genes has become one of the standard techniques in molecular systematics. These techniques have been used to compare nucleotides at homologous positions in the mitochondrial as well as in the nuclear DNA of different organisms. Every nucleotide position forms a character with four different options (adenine, thymine, guanine and cytosine). Particularly non-coding regions are part of analyses, since they do not underly a selective pressure. However, this method is limited by base composition similarities (Schmitz et al., 2002a) or when identical characters arise parallel in species without common ancestor (homoplasy). To reconstruct phylogenetic trees from nucleotide data different statistical models can be used like Maximum-Parsimony (MP), Maximum-Likelihood (ML), Neighbor-Joining (NJ) and Bayesian inference (Felsenstein, 1978, 1981; Saitou and Nei, 1987; Huelsenbeck and Ronquist, 2001). Further statistical methods like Bootstrapping (Felsenstein, 1985), Jackknifing (Farris et al., 1996) and Bayesian posterior probabilities (Huelsenbeck and Ronquist, 2001) can estimate the statistical support for the obtained tree topologies.

The choice of marker genes depends on the analysed evolutionary time window. The more variable the gene is, the more recent should be the analysed phylogenetic relationships. Ribosomal genes, which are relatively conserved, can be used for analysing splits more than 100 mya. Mitochondrial DNA has a constant mutation rate, is inherited maternally and does not recombine. Mutations accumulate 5-10 times faster than in nuclear DNA (Brown et al., 1982). Hence, mitochondrial DNA can rather be used for closer related taxa or population genetics. Analysing relative ancient splits using mitochondrial sequences could lead to unreliable results. The high copy number of mitochondrial genomes per cell (Cann et al., 1987) constitutes an advantage for analysing non-invasive samples (e.g. feces). However, the analyses of mitochondrial DNA can be hampered by 
possible misamplifications of nuclear pseudogenes (Collura and Stewart, 1995; Zischler et al., 1995; Mourier et al., 2001). As nuclear marker genes several intronic regions of different genes have been proven to be useful for various phylogenetic reconstructions (Flynn and Nedbal, 1998; Chaves et al., 1999; Hellborg and Ellegren, 2003; Yoder et al., 2003). Evolutionary processes could be affected by hybridization events between different subspecies, species or even genera. To reveal hybridization events it is appropriate to analyse marker genes with maternal (mitochondrial DNA), paternal (Ychromosomal DNA) as well as biparental (autosomal DNA) inheritance. Another general problem in these evolutionary analyses is incomplete lineage sorting (differential sorting of ancestral polymorphisms into the progeny lineages), particularly when analysing rapid radiations.

\subsubsection{Mobile elements - temporal landmarks in evolution}

As a result of sequencing numerous eucaryote genomes within the last years it became obvious that not more than $5 \%$ of the genome codes for proteins. In fact, more than $40 \%$ of the human and non-human genomes consist of a variety of repetitive sequences with apparent no global function (Smit and Riggs, 1995; Lander et al., 2001; Gibbs et al., 2007). These genome components represent remnants of elements, which have the ability to transport or duplicate themselves to other genomic locations. It is assumed that these mobile elements play a major role in shaping genomes during evolution. Mobile elements can be divided into two distinct groups, based on their mobility process. DNA transposons move to their target site by a cut and paste mechanism via a DNA intermediate, whereas retrotransposons mobilize through an RNA intermediate and a copy and paste mechanism (Shedlock and Okada, 2000; Kazazian, 2004; Shedlock et al., 2004). DNA transposons have been shown to be inactive for the last 40 million years, at least in primates (Pace and Feschotte, 2007).

Retrotransposons can further be divided into autonomous elements, which encode for their own replication machinery, and non-autonomous elements, which depend on the enzymatic machinery provided by other elements. The former group includes non-LTR (long terminal repeat) elements (LINEs, long interspersed elements) and LTR elements (retroviruses), whereas latter includes SINEs (short intersperse elements) (Shedlock and Okada, 2000; Kazazian, 2004; Shedlock et al., 2004). The retropositionary process is initiated by generating a RNA copy of the original element. This RNA intermediate is reverse transcribed into DNA by a process called target primed reverse transcription, followed by reintegration into a new genomic location. It is suggested that the retropositionary process of SINEs is mediated by a reverse transcriptase and endonuclease activity provided by LINEs (Luan et al., 1993; Feng et al., 1996; Jurka, 1997; Kazazian and Moran, 1998; Kajikawa and Okada, 2002; Kazazian, 2004). Furthermore, as a result of this process target site duplications (direct repeats) are generated.

SINEs are further divided into two different groups, either 7SL RNA or tRNA derived elements. Alu elements are primate-specific dimeric 7SL RNA-derived elements and first appear at the base of in primate evolution about 65 mya. They emerge to the elements with the highest copy number (at least one million in the genome of macaque and human) and a broad taxonomic distribution (Lander et al., 
2001; Li et al., 2001; Gibbs et al., 2007). With a length of about 300 basepairs (bp) they form the majority of SINEs and can be found mainly in untranslated and intergenic regions and introns (Deininger and Batzer, 1993). During primate evolution, Alu elements propagated in the genome in a chronological wave-like fashion and can be classified into three different temporally active subfamilies depending on specific mutations: AluJ (oldest), AluS (intermediate) and AluY (youngest) (Batzer et al., 1996). Therefore it was suggested that only a small subset of Alu elements, referred to as master-genes, are retropositional active in certain time windows (Deininger et al., 1992). However, this "master gene hypothesis" is in contrast to the "multiple source gene model" of Schmid and Maraia (1992), which assumed that also progeny Alu elements have the same ability for retroposition.

Regarding the size of a primate genome, the coincidence of an orthologous, independent and irreversible insertion of an Alu element into the same locus is insignificant. Furthermore the true orthology can be verified by their flanking direct repeats and their subfamily. Precise removal is extremely unlikely in multiple genomes and was only shown to be caused by recombination of perfect direct repeats (van de Lagemaat et al., 2005). These exact deletions were reported solely for few cases from very recent retroposed elements (Sen et al., 2006). Consequently, the lack of an Alu element at a certain locus can be considered to be the ancestral state and it is possible to determine a common ancestor by a shared insertion. However, SINE based phylogenetic analyses have some disadvantages. The major limitation is the uneven distribution of an Alu element into progeny lineages, caused by a non-fixed and unspecific insertion in a population, a phenomenon called incomplete lineage sorting. However, if there are no contradicting results, a significant support for a specific branching will be obtained by the detection of at least three independent insertions per branch (Waddell et al., 2001). Moreover, Alu elements are uninformative concerning estimation of divergence times, even though subfamilies give a hint for that, due to their activity in a certain time window (Schmitz et al., 2005).

Based on the characteristics outlined above, SINE insertions represent an effective alternative way to reconstruct phylogenies. This is well reflected in a variety of studies (Shimamura et al., 1997; Takahashi et al., 1998; Nikaido et al., 1999; Takahashi et al., 2001; Roos et al., 2004; Nishihara et al., 2005; Ray et al., 2005; Schmitz et al., 2005; Xing et al., 2005; Kriegs et al., 2006; Piskurek et al., 2006; Kriegs et al., 2007; Matveev et al., 2007; Xing et al., 2007), which for example provide decisive evidence for the position of whales within Cetartiodactyla (Nikaido et al., 1999), the phylogenetic relationships among strepsirrhine genera (Roos et al., 2004) or the monophyly of several mammalian clades (Kriegs et al., 2006). 


\subsection{Mobile Elements in Primate Evolution}

Considering the advantages and features of mobile elements as a phylogenetic marker system outlined above, several studies, mainly using Alu elements, were already performed to resolve several important questions in primate evolution (Schmitz et al., 2001; Salem et al., 2003; Schmitz and Zischler, 2003; Roos et al., 2004; Ray et al., 2005; Schmitz et al., 2005; Xing et al., 2005; Xing et al., 2007). Aim of this thesis was to use this technique to answer the most important phylogenetic issues in primate evolution. Hence, for an overview recent progress in mobile element based primate phylogeny and remaining questions in primate evolution will be outlined in order of branching events.

As already mentioned in Chapter 1.1, several studies based on retroposons provided evidence for the position of primates as a monophyletic group within the cohort Archonta, the position of the tarsier and the major division into Strepsirrhini and Haplorhini (Kuryshev et al., 2001; Schmitz et al., 2001; Schmitz et al., 2002a; Schmitz and Zischler, 2003; Schmitz et al., 2005).

Strepsirrhine primates are divided into Lemuriformes and Lorisiformes. Ongoing questions were the origin of strepsirrhines, whether Madagascar and Asia were colonized by single immigration events and the affiliations of the aye-aye (Daubentonia) among strepsirrhines. Roos et al. (2004) performed a SINE based study and inferred a strong supported phylogenetic tree. They demonstrated that lorisiforms and lemuriforms form reciprocal monophyletic groups, which argued for single colonization events and for an African origin of strepsirrhines. This scenario is also supported by Yoder (1996) and Karanth et al. (2005). The results proved a sister relationship between the aye-aye and the remaining Lemuriformes. Herke et al. (2007) partially confirmed the study of Roos et al. (2004). However, both studies were not able to elucidate confidently further branching orders among Lemuriformes families and the relationships within the Galagidae.

Phylogenetic relationships among New World monkey genera are highly disputed, because morphological and molecular studies reveal contradicting results. The branching order of the families Cebidae, Atelidae and Pitheciidae has not been confidently resolved using classical techniques. Hence, two SINE based analyses were performed (Singer et al., 2003; Ray et al., 2005). The study of Singer et al. (2003) merely indicated a monophyletic origin for all New World monkeys, for the Cebidae and for the Callithrichinae. Ray et al. (2005) confirmed these results and provided strong evidence for a closer affiliation of Cebidae and Atelidae and a basal position of Pitheciidae. Furthermore the study resolved some phylogenetic relationships within Pitheciidae and Atelidae. While the first study was not comprehensive enough, the second study did not include all genera. Evidence for the phyletic unity of the Cebidae is still lacking. Moreover there are no SINE based studies regarding the phylogenetic relationships within the Callitrichinae and their affiliations to the genera Saimiri, Aotus and Cebus at hand. 


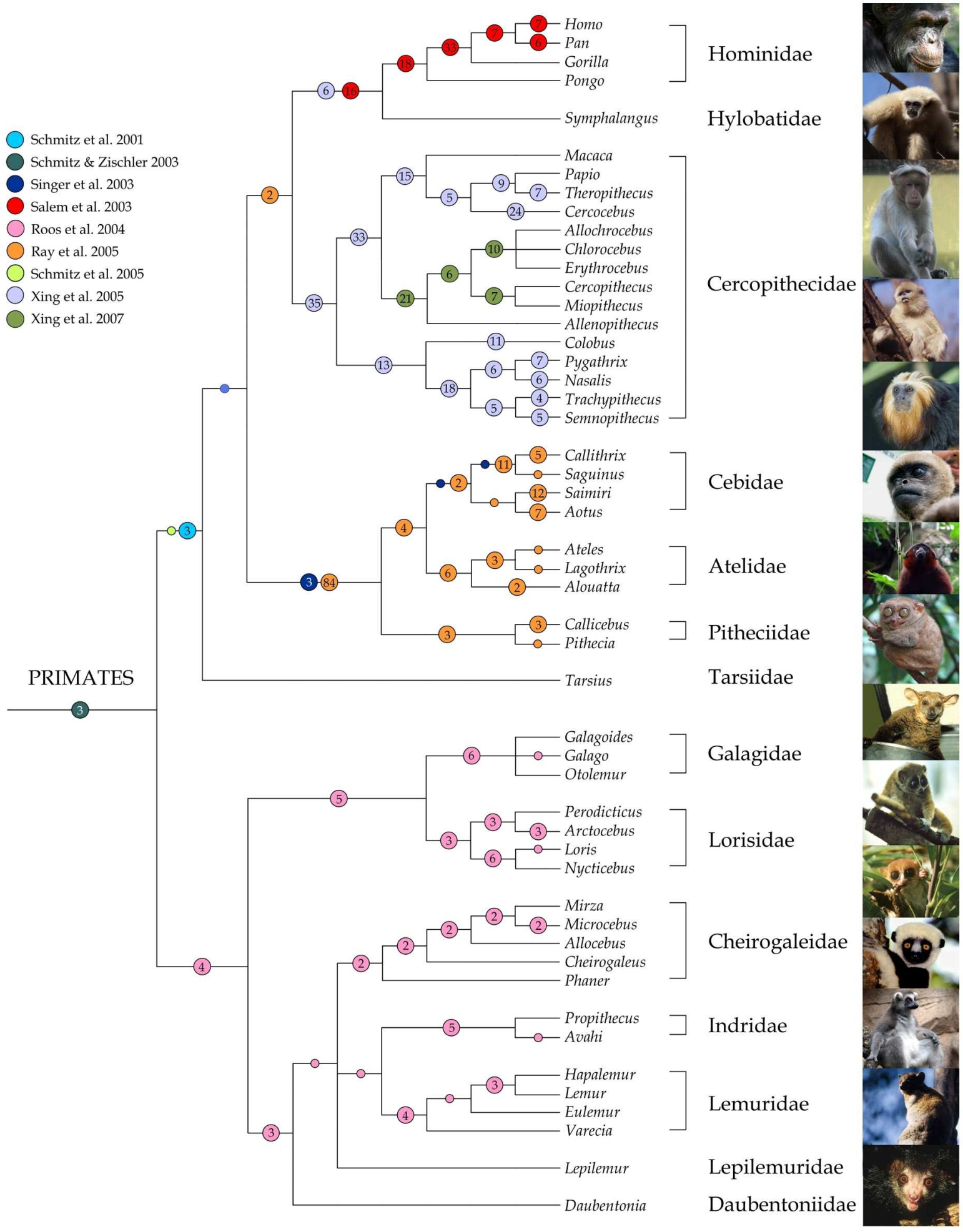

Figure 1.0: Primate phylogeny based on published mobile elements. Numbers of integrations per branch are indicated by small circles (one integration) and large circles (number of integrations inside)

Within the Old World monkeys there is little disagreement between morphological and molecular data regarding the higher-level phylogenetic affiliations within Cercopithecinae (tribes and subfamilies). Crucial disagreements in Old World monkey phylogeny can be found in colobine monkeys. This concerns especially the question of respective monophyly of Asian and African clades 
and several genus affiliations. Two studies based on SINE insertions were performed to resolve questions in Old World monkey phylogeny (Xing et al., 2005; Xing et al., 2007). The first study provides strong evidence for the major clades in colobine monkeys (although solely Colobus was tested as African genus) and cercopithecines and was in agreement with former molecular or morphological studies (Delson, 1992; Goodman et al., 1998; Zhang and Ryder, 1998; Page et al., 1999; Page and Goodman, 2001; Sterner et al., 2006). They provided evidence for the monophyly of Papionini and Cercopithecini. Furthermore Papio was confirmed as sister genus to Theropithecus. Cercocebus forms the sister clade to Papio and Theropithecus and Macaca to all of them. However, Lophocebus, Mandrillus and the recently described Rungwecebus were not implemented in their study. Phylogenetic relationships within the Asian colobines were conclusively shown with Trachypithecus and Semnopithecus (Trachypithecus vetulus) as well as Pygathrix and Nasalis as sister clades. However, most exciting and interesting questions in colobine evolution, e.g. monophyly of African and Asian colobines, could not be examined, since relevant genera were not included. The second study of Xing et al. (2007) focused solely on the Cercopithecini and supported the theory that all terrestrial taxa (Cercopithecus lhoesti, Erythrocebus patas, Chlorocebus aethiops) form a monophyletic clade resulting in a paraphyly of the genus Cercopithecus. Furthermore the study documented the basal position of Allenopithecus within this tribe. Questionable is, whether further SINE analyses would be the right way to resolve the few remaining questions in Cercopithecinae, since most splitting events are assumed to be radiation-like or too recent (Schmitz et al., 2005). As mentioned in Chapter 1.1 the phylogenetic relationships within Hominidae were additionally resolved by a study based on mobile elements as well (Salem et al., 2003). However, there is no mobile element based study concerning relationships among gibbons at hand, because recent molecular and chromosomal analyses indicate that gibbons could be divided into four different genera (Roos and Geissmann, 2001).

Although many issues were addressed and solved by several SINE based studies, crucial questions in primate phylogeny remain. This is due to the fact that nearly all studies lack essential genera. Hence, the first part of this thesis addresses the remaining questions in New World monkey phylogeny and is presented and discussed in Chapter 2. In the frame of this study a SINE insertion distinguishing Saimiri boliviensis boliviensis from Saimiri sciureus was detected, two species, which are both common in zoos and biomedical research. The relevance of this finding is presented and discussed in Chapter 3. As outlined above there are still crucial disagreements in the evolutionary history of colobine monkeys. Chapter 4 and 5 are dedicated to these fundamental issues. 


\section{Retropositional events consolidate the branching order among New World monkey genera}

Martin Osterholz ${ }^{1^{*}}$, Lutz Walter ${ }^{1,2}$, and Christian Roos ${ }^{1,2}$

1Primate Genetics and ${ }^{2}$ Gene Bank of Primates, German Primate Center, Kellnerweg 4, 37077

Goettingen, Germany

${ }^{*}$ Corresponding author

submitted to Molecular Phylogenetics and Evolution 


\begin{abstract}
Due to contradicting relationships as obtained from various morphological and genetic studies, phylogenetic relationships among New World monkey genera are highly disputed. In the present study, we analyzed the presence/absence pattern of 128 SINE integrations in all New World monkey genera. Among them, 70 are specific for only a single genus, whereas another 18 are present in all New World monkey genera. The 40 remaining insertions are informative to elucidate phylogenetic relationships among genera. Several of them confirm the monophyly of the three families Cebidae, Atelidae and Pitheciidae as well as of the subfamily Callithrichinae. Further markers provide evidence for a sister grouping of Cebidae and Atelidae to the exclusion of Pitheciidae as well as for relationships among genera belonging to Callithrichinae and Atelidae. Although a close affiliation of Saimiri, Aotus and Cebus to Callithrichinae is shown, the relationships among these three genera are not resolved due to contradicting relationships as obtained from three independent insertions.
\end{abstract}

\title{
2.1 Introduction
}

New World monkeys, infraorder Platyrrhini, diverged from Old World monkeys about 40 million years ago (Goodman et al., 1998), and are distributed from northern Argentina to Mexico. Their common origin is widely accepted and confirmed by morphological features like dental, cranial or placental attributes as well as by molecular data (Hofer, 1976; Rosenberger, 1977; Luckett, 1980; Schneider et al., 1993; Horovitz and Meyer, 1995; Porter et al., 1995; Porter et al., 1997; Horovitz et al., 1998; Singer et al., 2003; Ray et al., 2005). New World monkeys represent a highly diverse group with an extensive morphological and ecological adaptive diversity, manifested in the presence of up to thirteen sympatric species (Fleagle, 1988). Accordingly, reconstructing phylogenetic relationships among platyrrhine genera is difficult.

Most recent classifications arrange New World monkeys into three families (Cebidae, Atelidae and Pitheciidae) with 14 to 15 genera (Goodman et al., 1998; Opazo et al., 2006), but alternative classifications with only two (Napier and Napier, 1967) or even four families (Groves, 2001) were also proposed. The branching order among the three families is still disputed and even recent molecular analyses were not able to confidently resolve it, mainly due to incongruences between different marker systems (Schneider et al., 1993; Harada et al., 1995; Schneider et al., 1996; Goodman et al., 1998; Canavez et al., 1999; Steiper and Ruvolo, 2003; Ray et al., 2005; Opazo et al., 2006). Questioned are also the phylogenetic relationships of genera within the Cebidae subfamily Callithrichinae and the familiy Atelidae as well as the positions of the genera Saimiri, Cebus, Aotus and Callicebus.

Within the Callithrichinae, most morphological studies agree in placing Callimico as the basal genus, whereas they differ in placing Saguinus either as a sister genus to Callithrix, or to a Callithrix + Leontopithecus clade (Rosenberger, 1981; Ford, 1986; Kay, 1990). However, molecular studies provide evidence for a close affiliation of Callithrix and Callimico and a basal position of Saguinus (Pastorini et al., 1998; Canavez et al., 1999; Chaves et al., 1999; von Dornum and Ruvolo, 1999; Singer et al., 2003). Other molecular studies suggest two clades with one comprising Callithrix and Callimico and the other 
Leontopithecus and Saguinus (Schneider et al., 1993; Harada et al., 1995; Porter et al., 1997; Goodman et al., 1998).

Within Atelidae, various relationships among its four genera were proposed. According to Rosenberger (1981), Ateles and Brachyteles form a clade with Lagothrix as sister and Alouatta as basal genus. Kay (1990) defined two monophyletic clades (Alouatta + Brachyteles and Lagothrix + Ateles), while Ford (1986) proposed an unresolved trichotomy between Lagothrix, Ateles and Brachyteles, with Alouatta representing their sister genus. Most molecular studies agree in a close relationship between Lagothrix and Brachyteles, followed by Ateles and Alouatta (Schneider et al., 1993; Harada et al., 1995; Porter et al., 1997; Goodman et al., 1998; Horovitz et al., 1998; Canavez et al., 1999; von Dornum and Ruvolo, 1999), however, an unresolved relationship between Brachyteles, Lagothrix and Ateles is also proposed (Collins, 2004).

Highly disputed are the phylogenetic positions of the genera Saimiri, Cebus, Aotus and Callicebus among New World monkeys. Rosenberger (1981) and Ford (1986) proposed a sister grouping of Callicebus and Aotus as well as of Saimiri and Cebus, but they differ in positioning them among platyrrhines. Saimiri + Cebus is either the most basal clade (Ford, 1986) or forms a sister clade to Callithrichinae (Rosenberger, 1981). Aotus + Callicebus is either a sister clade to Callithrichinae, Atelidae and Pitheciidae (Ford, 1986) or solely to the latter (Rosenberger, 1981). According to Kay (1990), Callicebus is the most basal genus among platyrrhines followed by Cebus, whereas Saimiri and Aotus form a sister clade to Callithrichinae. Molecular studies support a grouping of Callicebus with Pitheciidae and of Cebus, Saimiri and Aotus with Callithrichinae in the family Cebidae (Harada et al., 1995; Porter et al., 1997; Horovitz et al., 1998; Canavez et al., 1999; von Dornum and Ruvolo, 1999; Steiper and Ruvolo, 2003). In all these studies, a Cebus + Saimiri clade is depicted, while the position of Aotus remains disputed. Some suggest an unresolved position of Aotus in a Cebidae polytomy (Schneider et al., 1993; von Dornum and Ruvolo, 1999) or a basal position among Cebidae (Horovitz et al., 1998). Alternatively, Aotus is placed as as sister taxon to Cebus + Saimiri (Prychitko et al., 2005; Opazo et al., 2006).

As indicated above, the phylogenetic relationships among New World monkey families and genera are far away from being resolved. Since morphological and molecular studies seem to be not appropriate to tackle all these issues, Alu integrations were applied as cladistic markers (Singer et al., 2003; Ray et al., 2005). Alus as primate-specific SINE (Short INterspersed Elements) elements are a class of retrotransposons integrating via an RNA intermediate into the genome (Okada, 1991). The integration of a SINE at a new locus is irreversible and precise excision is highly unlikely (Shedlock and Okada, 2000; van de Lagemaat et al., 2005). Therefore, absence of an insertion at a certain locus can supposed to be the ancestral state and due to its fixation in the genome, a common ancestor can be determined by a shared derived insertion. Furthermore, a true orthology can be verified and homoplasy can be excluded by analysing their flanking direct repeats (Schmitz et al., 2005). Consequently, SINE insertions represent a powerful molecular cladistic tool for reconstructing 
phylogenetic relationships (Shimamura et al., 1997; Takahashi et al., 2001; Roos et al., 2004; Ray et al., 2005; Xing et al., 2005; Kriegs et al., 2006; Osterholz et al., 2008).

In the present study, we analyzed the presence/absence pattern of SINE insertions in all platyrrhine genera. To detect new loci, a computational as well as a subtractive hybridization approach was applied. Moreover, we tested genera, which have not been analyzed in an earlier study (Ray et al., 2005), but are crucial to depict the exact location of respective SINE insertions in the platyrrhine tree.

\subsection{Methods}

\subsubsection{Computational approach}

Sequences from Bacterial Artificial Chromosome (BAC) clones from five New World monkeys (Callithrix jacchus, Saimiri boliviensis, Aotus nancymaae, Ateles geoffroyi and Callicebus moloch) were obtained from GenBank database (Supplementary table 2.1). To identify SINEs of interest, we scanned the GenBank database for orthologous BAC clones from different genera. These were aligned using MAFFT software (Katoh et al., 2005) and further scanned for SINEs using RepeatMasker (www.girinst.org) (Jurka et al., 2005). For further analyses, only insertion loci specific for at least one New World monkey species were selected. The orthology of lineage specific insertions were confirmed by checking direct repeats. At least $400 \mathrm{bp}$ of flanking sequences were then compared with the genomes of human, chimpanzee and rhesus macaque using BLAT search of the UCSC Genome Browser (Kent et al., 2002). Based on this information, locus-specific oligonucleotide primers in conserved flanking regions of the insertion were designed for further PCR analyses.

\subsubsection{Subtractive hybridization approach}

As second approach, a subtractive hybridization was applied, following methods as described in Osterholz et al. (2008), Mamedov et al. (2005) and Diatchenko et al. (1996). We conducted two subtractive hybridizations. In the first, Lagothrix was selected as tracer and Saimiri as driver, whereas in the second, Pithecia and Chiropotes were applied as tracer and Ateles as driver. Genomic DNA of tracer and driver was digested with RSAI (Fermentas), and subsequently, the adapters AdapA1/AdapAA1 (5'- TGTAGCGTGAAGACGACAGAAAGGGCGTGGTGCGGAGGGCGGT-3' / 5'- ACCGCCCTCCG-3') and AdapA2/AdapAA2 (5'TGTAGCGTGAAGACCTGTCTTAGGGCGTGGTGGCCAGGGCCGT-3' / 5'- ACGGCCCTGGC-3') were ligated to the tracer fragments. Each of $\sim 15 \mathrm{ng}$ tracerA1 and tracerA2 were hybridized with $\sim 1,500 \mathrm{ng}$ driver for $20 \mathrm{~h}$ at $60^{\circ} \mathrm{C}$. $2 \mu \mathrm{l}$ of the hybridization result was used as template to amplify solely tracer fragments using the adapter primers A1 (5'-TGTAGCGTGAAGACGACAGAA-3') and A2 (5'-TGTAGCGTGAAGACCTGTCTT-3'). The PCR program consisted of a pre-extension step at $72^{\circ} \mathrm{C}$ 
for $6 \mathrm{~min}$ to fill in adaptor ends, followed by 25 cycles, each with a denaturation step at $95^{\circ} \mathrm{C}$ for $1 \mathrm{~min}$, annealing at $60^{\circ} \mathrm{C}$ for $1 \mathrm{~min}$ and extension at $72^{\circ} \mathrm{C}$ for $2 \mathrm{~min}$. To enrich fragments with Alu insertions, a semi-nested PCR was added using either primer A1 or A2 and the Alu-specific AluS (5'GGAGAATCGCTTGAACCCGGGA-3') oligonucleotide. The PCR products were separated on agarose gels and fragments over $500 \mathrm{bp}$ were excised from the gel. After purification, the fragments were cloned into the pGEMTeasy vector (Promega) and transformed into electro-competent TOP10 cells (Invitrogen). Bacterial clones were collected in 96-well microtiter plates and re-screened via PCR with the primers A1 or A2 and AluS. Positive clones were sequenced and analyzed with RepeatMasker to identify repeat structures. To identify orthologous sequences in human, chimpanzee or rhesus macaque, sequences were subjected either to a BLAST (NCBI) or a BLAT (UCSC) search. Based on generated alignments, conserved locus-specific primers were designed, with the forward primer occupying a region 5 -end upstream of the insertion site. Due to the absence of the 3 '-end downstream sequence in the tested new world monkey species, reverse primers were constructed solely on the basis of human, chimpanzee and rhesus macaque sequences.

\subsubsection{Testing the presence/absence of insertions in New World monkeys}

Samples from various New World monkeys were collected in European institutions: Alouatta caraya, Cacajo calvus, Callimico goeldii, Callithrix pygmaea, Chiropotes albinasus $x$ satanas, Leontopithecus chrysomelas (Cologne zoo), Aotus azarae (Gettorf zoo), Saguinus imperator, Samiri sciureus (German Primate Center), Cebus apella (Romagne zoo), Lagothrix lagothricha (Basel zoo), Ateles fusciceps (Landau zoo) and Pithecia pithecia (Dortmund zoo). Total genomic DNA from blood or tissue material was extracted with the Qiagen DNeasy Blood \& Tissue Kit. DNA from Brachyteles arachnoides was provided by Stefan Müller (University of Munich).

To examine the presence/absence pattern of SINE insertions, we first tested only one representative of each family using locus-specific primers as listed in supplementary table 2.2. Based on this information, further relevant genera were examined. PCR conditions for all reactions were identical and included an initial denaturation step at $94^{\circ} \mathrm{C}$ for $2 \mathrm{~min}$., followed by 40 cycles each with of one minute of denaturation at $94^{\circ} \mathrm{C}$, annealing at $58^{\circ} \mathrm{C}$ and extension at $72^{\circ} \mathrm{C}$. At the end, a final extension step at $72^{\circ} \mathrm{C}$ for $5 \mathrm{~min}$. was added. Besides newly detected integration loci, we also tested 29 insertions described by Ray et al. (2005) (Supplementary table 2.2), which have not been tested in all relevant genera. PCR conditions for these amplifications were conducted as described in Ray et al. (2005). Results of the amplifications were checked on $1 \%$ agarose gels and further processed with the Wizard gel purification kit (Promega). To verify the orthology of loci and insertions, PCR products were sequenced on an ABI3100-Avant sequencer using the Big Dye Terminator Cycle Sequencing Kit (Applied Biosystems). Sequences were edited using BioEdit software (Hall, 1999) and aligned manually. Sequences generated in the present study are available in Genbank (Accession nos. $x x x x x x x-x x x x x x x)$. 


\subsection{Results}

In total, 128 integrations were studied (Fig. 2.1, supplementary table 2.2). 21 of them were obtained from the subtractive hybridization approach, whereas 78 were detected by BAC clone screening. 29 integrations were derived from a previous study (Ray et al., 2005). With the exception of two Atelinaespecific insertions described by Ray et al. (2005), which provided ambiguous results for Brachyteles, all others showed a clear presence/absence pattern of integrations. 70 of them represent autapomorphies, which are only present in a single genus and another 18 insertions are present in all New World monkeys. Since these markers provide no information on phylogenetic relationships among New World monkey genera, they were examined only on PCR level without confirming the integration site by sequencing. Finally, 38 insertions were identified which can be applied to reconstruct phylogenetic relationships among platyrrhine genera.

The monophyly of the family Atelidae is confirmed by five insertions. Relationships within the family are not well resolved, although at least three insertions were detected which are present in Lagothrix, Brachyteles and Ateles and absent in Alouatta. A common origin of the four Pitheciidae genera Pithecia, Cacajo, Chiropotes and Callicebus is confirmed by four integrations, but no loci are available which resolve relationships among them. Seven integrations are present in all tested Cebidae representatives. To resolve relationships among Cebidae genera, several informative insertions are available. These support the monophyly of the subfamily Callithrichinae (five integrations) and a Callithrix + Callimico clade (seven insertions) as well as of a clade consisting of Callithrix, Callimico and Leontopithecus to the exclusion of Saguinus (three insertions). Contradicting relationships were obtained for the three Cebidae genera Saimiri, Cebus and Aotus. Whereas one insertion is present in Saimiri and Aotus, another two integrations support a Saimiri + Cebus and a Cebus + Aotus clade. Relationships among the three platyrrhine families are not well resolved by our study, but at least one insertion was detected which is present in Cebidae and Atelidae and absent in Pitheciidae.

\subsection{Discussion}

In general, all phylogenetic relationships proposed by Ray et al. (2005) and Singer et al. (2003) were confirmed in the present study, however, both previous studies did not include all genera and/or described only a few integrations. In contrast, we included all New World monkey genera and analyzed the presence/absence pattern of a relatively large number of SINE integrations.

Among 128 insertions examined, 18 are present in all New World monkey genera. In combination with the 84 loci detected by Ray et al. (2005) and three by Singer et al. (2003), now 105 independent SINE insertions provide evidence for a common origin of the infraorder.

The proposed diversification of New World monkeys into the three families Cebidae, Atelidae and Pitheciidae (Goodman et al., 1998; Opazo et al., 2006) is supported by several insertions. We detected four integrations on the branch leading to all Pitheciidae genera. Together with three 


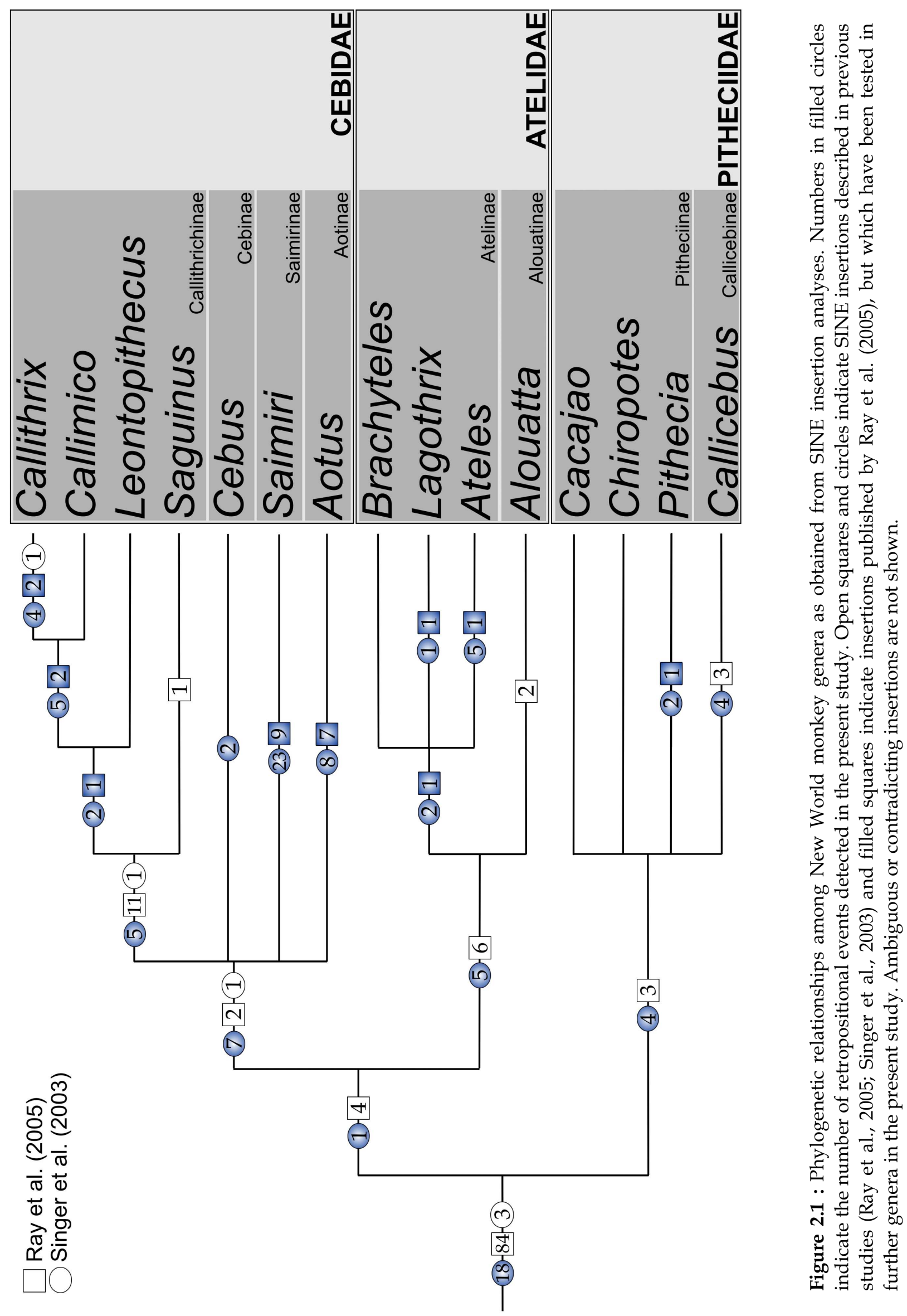


retropositional events described by Ray et al. (2005), a monophyletic Pitheciidae clade is confirmed. Five and seven integrations are present in all Atelidae and Cebidae genera, respectively. Combining them with earlier data (Ray et al. 2005; Singer et al. 2003), eleven and ten integration sites support the monophyly of both families.

The phylogenetic relationships among these three families are highly disputed. Morphological studies support a common origin of Atelidae and Pitheciidae (Rosenberger, 1981, 1984; Ford, 1986). Alternatively, Cebidae group with Atelidae to the exclusion of Pitheciidae (Kay, 1990). Molecular analyses based on mitochondrial sequence data (Horovitz and Meyer, 1995) or nuclear data (Steiper and Ruvolo, 2003) were not able to solve the relationships among them, although a grouping of Atelidae and Cebidae is assumed (Opazo et al., 2006). In the present study, we detected one insertion, which indicates an Atelidae + Cebidae clade. Together with four insertions provided by Ray et al. (2005), there is now clear evidence that Pitheciidae form the basal family among New World monkeys and that Atelidae and Cebidae represent sister lineages.

As mentioned above, the monophyletic origin of the Cebidae family including Callithrichinae and the genera Cebus, Saimiri and Aotus is confirmed by ten integrations, which is concordant with other molecular studies (Goodman et al., 1998; Singer et al., 2003; Steiper and Ruvolo, 2003; Ray et al., 2005; Opazo et al., 2006). The common origin of Callithrichinae is also supported by morphological investigations (Rosenberger, 1981, 1984; Ford, 1986; Kay, 1990), but they differ in positioning Cebus, Saimiri and Aotus among New World monkeys. Although all molecular studies clearly confirm a grouping of these genera together with Callithrichinae, the relationships among them remain unresolved. We detected three independent insertions concerning the phylogenetic relationships of Saimiri, Aotus and Cebus. The first one supports a sister grouping of Aotus and Cebus in a polytomy with Saimiri and Callithrichinae. However, the second one indicates a close affiliation of Aotus to Saimiri in a polytomy with Cebus and Callithrichinae and the third one shows the third possible combinations with Saimiri and Cebus as sister lineages. These contradicting results might be explained by incomplete lineage sorting of ancestral polymorphisms, which are common when species diverge from each other within a relative short time period. Support for such a radiation-like diversification of the four lineages is gained from the small number of observed informative and the large number of autapomorphic markers. Further support for an adaptive radiation is also provided by the relative short time period separating these four lineages as estimated from molecular sequence data (Opazo et al., 2006; Schrago, 2007).

In contrast, the monophyly and relationships within Callithrichinae are strongly supported by several integrations. We detected five integrations shared by all Callithrichinae. Together with twelve earlier described insertions (Ray et al., 2005; Singer et al., 2003), a common origin of the Callithrichinae subfamily is strongly supported. Both molecular and morphological studies agree in this respect, but the branching order within the subfamily is disputed - mainly whether Saguinus, Leontopithecus or Callimico form the basal genus (Rosenberger, 1981; Ford, 1986; Goodman et al., 1998; Pastorini et al., 1998; Schneider et al., 2001; Singer et al., 2003). Especially, the position of Callimico was challenging. 
Although most studies placed the genus as basal among Callithrichinae (Napier and Napier, 1967; Szalay and Delson, 1979), others included it either in Cebidae (Martin, 1990) or in its own family Callimiconidae (Hershkovitz, 1977). Molecular studies support a classification among Callithrichinae and specifically as sister lineage to Callithrix (Harada et al., 1995; Horovitz and Meyer, 1995; Schneider et al., 1996; Porter et al., 1997; Pastorini et al., 1998; Canavez et al., 1999; Chaves et al., 1999; von Dornum and Ruvolo, 1999; Singer et al., 2003). In the present study, we obtained seven insertions shared by Callimico and Callithrix, confirming their sister group relationship. Three further markers unite Callithrix, Callimico and Leontopithecus, therefore suggesting that Saguinus forms the basal form among all Callithrichinae. This arrangement is in agreement with several molecular studies (Schneider et al., 1996; Chaves et al., 1999; Singer et al., 2003; Opazo et al., 2006), however, contradicts with the proposed sister grouping of Saguinus and Leontopithecus (Schneider et al., 1993; Harada et al., 1995; Porter et al., 1997; Goodman et al., 1998).

The monophyly of Atelidae is supported by five retropositional events. With the insertions detected by Ray et al. (2005), a common origin of the four Atelidae genera Alouatta, Ateles, Lagothrix and Brachyteles is now confirmed by eleven integrations. Combining morphological and molecular studies, this arrangement seems to be evident (Rosenberger, 1981; Ford, 1986; Kay, 1990; Porter et al., 1995; von Dornum and Ruvolo, 1999; Opazo et al., 2006; de Lima et al., 2007). Moreover, three markers are present in Ateles, Lagothrix and Brachyteles, but absent in Alouatta, supporting other molecular (Schneider et al., 1993; Harada et al., 1995; Porter et al., 1997; Goodman et al., 1998; Horovitz et al., 1998; Canavez et al., 1999; von Dornum and Ruvolo, 1999) and some morphological investigations (Rosenberger, 1981; Ford, 1986). Accordingly, the splitting of the family into the two subfamilies Alouatinae with the single genus Alouatta and Atelinae including the remaining three genera is appropriate. The relationships among the three Atelinae genera are not resolved, due to the lack of informative markers.

Four insertions support the monophyly of Pitheciidae, including Callicebus. The position of this unique genus was highly disputed, mainly due to morphological autapomorphies (Rosenberger, 1981; Ford, 1986; Kay, 1990), but molecular data clearly supported its close affiliations to Pithecia, Chiropotes and Cacajo (Goodman et al., 1998; Steiper and Ruvolo, 2003; Opazo et al., 2006). Based on our four integrations and three already described ones (Ray et al., 2005), the unity of the family is confirmed. However, no further markers, which elucidate the branching order among the four Pitheciidae genera were detected.

\subsection{Conclusions}

By analyzing the presence/absence pattern of 128 SINE insertions, the present study deepens our knowledge on the phylogenetic relationships among New World monkey genera. Based on our and other SINE data, a common origin of the three families Cebidae, Atelidae and Pitheciidae can be regarded as settled as well as a sister grouping of Cebidae and Atelidae to the exclusion of Pitheciidae. 
Although the monophyly of Cebidae and its subfamily Callithrichinae is confirmed, the phylogenetic positions of Saimiri, Aotus and Cebus in the Cebidae clade remain unresolved. Due to the obtained contradicting relationships, which might be affected by incomplete lineage sorting, further efforts have to be undertaken to solve this issue. Our results further provide evidence for the branching order among Callithrichinae genera as well as for a basal position of Alouatta among Atelidae. As indicated by this and earlier studies, retropositional events provide a powerful molecular cladistic tool to reconstruct reliable phylogenies, which form a solid platform for various behavioral, morphological and molecular investigations.

\section{Acknowledgements}

We are grateful to the staff of the zoos in Cologne, Dresden, Gettorf, Basel, Romagne, Landau and Dortmund and the German Primate Center (DPZ) for samples, as well as to Stefan Müller for providing DNA from Brachyteles arachnoides. Finally, we thank Christiane Schwarz and Nico Westphal for their excellent technical assistance in the laboratory. The work was financially supported by the DPZ. 


\section{A PCR-based marker to simply identify Saimiri sciureus and S. boliviensis boliviensis}

Martin Osterholz ${ }^{1}$, Jan Vermeer ${ }^{2}$, Lutz Walter ${ }^{1,3}$, and Christian Roos ${ }^{* 1,3}$

1Primate Genetics, German Primate Center, Kellnerweg 4, 37077 Goettingen, Germany

2La Vallée des Singes, Le Gureau, 86700 Romagne, France

${ }^{3}$ Gene Bank of Primates, German Primate Center, Kellnerweg 4, 37077 Goettingen, Germany

${ }^{*}$ Corresponding author

American Journal of Primatology 2008;70: 1-4. 


\begin{abstract}
Squirrel monkeys, mainly Saimiri sciureus and S. boliviensis, are common in zoos and widely used in biomedical research. However, an exact species identification based on morphological characteristics is difficult. Hence, several molecular methods were proposed, but all of them are expensive and require extensive laboratory work. In contrast, we describe an Alu integration, which is present in S. boliviensis boliviensis and absent in S. sciureus. Among analysed S. b. peruviensis specimens various presence / absence patterns of the integration were detected indicating that this study population might have originated from a natural hybrid zone. Based on the size of the Alu element $(\sim 300 \mathrm{bp})$, the presence / absence pattern of the integration can easily be traced by PCR and followed by agarose gel electrophoresis.
\end{abstract}

\title{
3.1 Introduction
}

Squirrel monkeys (genus Saimiri) are New World monkeys and belong to the family Cebidae. The genus is distributed, apart from some isolated populations in Costa Rica and Panama, in tropical forests of northern South America to Bolivia and central Brazil (Hershkovitz, 1984; Rowe, 1996; Groves, 2001). The taxonomic classification of squirrel monkeys changed several times with one to seven species and seven to sixteen subspecies (Elliot, 1913; Lönnerg, 1940; von Pusch, 1942; Hill, 1960; Hershkovitz, 1984; Thorington, 1985; Costello et al., 1993; Rowe, 1996; Groves, 2001). Based on morphological, acoustic, chromosomal, molecular and behavioral differences, most authors agree in distinguishing two different species groups, S. boliviensis including S. vanzolinii, and S. sciureus with $S$. ustus and S. oerstedii (Hershkovitz, 1984; Boinski and Newman, 1988; Moore et al., 1990; Rowe, 1996; Boinski and Cropp, 1998; Schreiber et al., 1998; Groves, 2001).

Squirrel monkeys, mainly S. sciureus and S. boliviensis, are common in zoos and widely used in biomedical research (Mittermeier et al., 1994; Vermeer, 1996). Accordingly, many breeding colonies exist, and in order to minimize inbreeding, animals are regularly exchanged among them. However, because the exact origin of founder animals is mainly unknown, the (sub)species identity of such animals is difficult to assess with morphological characteristics (Vermeer, 1996). As a result, several hybrids were produced between species or subspecies in recent decades (Vermeer, 1996; Schreiber et al., 1998). Although both S. sciureus and S. boliviensis are currently classified as only "least concern" (IUCN, 2007), pure breeding is of interest for conservation issues. In biomedical research, pure breed lineages are also important, because species differ in critical biological parameters as e.g. susceptibility to diseases (VandeBerg et al., 1990; VandeBerg and Williams-Blangero, 1997; Lavergne et al., 2003). However, since an accurate identification of species and hybrids based on external characteristics is difficult, methods not reliant on phenotype (e.g. molecular markers) should be applied. Some molecular tests based on allozyme and microsatellite polymorphisms or sequencing of marker genes have been proposed (VandeBerg et al., 1990; Silva et al., 1993; Boinski and Cropp, 1998; Schreiber et al., 1998; Cropp and Boinski, 2000; Lavergne et al., 2003), but all of them are expensive and time demanding. 
Short INterspersed Elements (SINE) represent a class of retrotransposons integrating via an RNA intermediate into the genome (Okada, 1991). The integration of a SINE at a new locus is irreversible and precise excision is highly unlikely (Shedlock and Okada, 2000; van de Lagemaat et al., 2005). Orthology can be verified and homoplasy can be excluded by tracing direct repeats which flank the integration (Schmitz et al., 2005). Alu elements are primate-specific SINEs and have been widely propagated in their genomes.

In this study, we tested the presence / absence pattern of an Alu insertion in 93 squirrel monkeys.

\subsection{Material \& Methods}

\subsubsection{Database approach}

Bacterial Artificial Chromosome (BAC) clones from various New World monkey species (S. $b$. boliviensis, Callithrix jacchus, Aotus nancymaae, Ateles geoffroyi and Callicebus moloch) were obtained from GenBank database. Using BLAT search, orthologous BAC clones were identified and aligned with MAFFT software (Katoh et al., 2005). Subsequently, Alu integrations were identified with RepeatMasker (Jurka et al., 2005). At least 400 bp sequence information from both sides of the integration site was selected and subjected to BLAT search to find orthologous loci in the genomes of Homo sapiens, Pan troglodytes and Macaca mulatta. Based on this information, conserved oligonucleotide primers were constructed, which bind in the flanking regions of the Alu insertion. In the frame of this study, one AluTa15 integration (SscSbo) was detected, which was present in S. b. boliviensis, but absent in other platyrrhines.

\subsubsection{Laboratory methods}

To further test the presence / absence pattern of the $S s c S b o$ integration, blood samples from 93 squirrel monkeys kept in European institutions were collected. The species identity of study specimens was determined by fur coloration and other external characteristics. Samples from phenotypically pure S. sciureus were provided by Dresden zoo $(n=2)$, Gettorf zoo $(n=5)$ and Schwerin zoo $(\mathrm{n}=1)$, phenotypically pure $S . b$. boliviensis from Mannheim zoo $(\mathrm{n}=2)$, Nuremberg zoo $(\mathrm{n}=3)$ and Romagne primate park $(\mathrm{n}=3)$ and $S . b$. peruviensis from Romagne primate park $(\mathrm{n}=53)$. Samples from animals, which were identified phenotypically as hybrids between S. boliviensis and S. sciureus were obtained from the German Primate Center $(n=14)$ and from Madrid zoo $(n=10)$. We have adhered to the guidelines for the use of animals in research and the legal requirements of Germany.

DNA from blood samples was extracted using the Qiagen DNA Mini kit. PCR amplifications were performed with the locus-specific oligonucleotide primers 5'-AGTTCCTCTCTACCTTGTACC-3' 
and 5'-GCCCTACTCTTGCATTAATGC-3'. The expected fragment lengths of the PCR product are $\sim 450$ and $\sim 750 \mathrm{bp}$ in the case of presence and absence of the AluTa15 integration, respectively (Fig. 3.1a). PCR conditions were $94^{\circ} \mathrm{C}$ initial denaturation for 2 minutes, followed by 40 cycles each with $94^{\circ} \mathrm{C}$ denaturation for 1 minute, $58^{\circ} \mathrm{C}$ annealing for 1 minute and $72^{\circ} \mathrm{C}$ extension for 1 minute. The final extension step at $72^{\circ} \mathrm{C}$ was performed for 5 minutes. Results of PCR amplifications were analysed using a $1 \%$ agarose gel. To confirm the orthology of the integration, PCR products from each one individual of S. sciureus and S. b. boliviensis were sequenced. Therefore, PCR products were excised from the gel and purified with the Wizard gel purification kit (Promega). Sequencing reactions were run on an ABI 3100-Avant sequencer using the Big Dye Terminator Cycle Sequencing Kit (Applied Biosystems), and the primers mentioned above. To confirm the orthology of the integration, sequences were edited and manually aligned in BioEdit (Hall, 1999).

\subsection{Results}

In an alignment including BAC clones from S. b. boliviensis (AC188239), C. jacchus (AC188222), A. geoffroyi (AC188259) and C. moloch (AC188270), an AluTa15 (Ray and Batzer, 2005) insertion was detected, which is present in S. b. boliviensis and absent in the remaining three taxa. To further check the presence and absence of the integration, conserved primers binding in the flanking region of the insertion were constructed and tested in a panel of various New World monkeys. Interestingly, all tested species including S. sciureus showed an absence of the Alu insertion at that locus. Consequently, further squirrel monkey individuals were examined to test whether the integration is specific for $S$. boliviensis. Among the 93 squirrel monkeys examined, in 39 individuals the integration is present $(+/+)$, whereas in 18 the integration is absent (-/-). In another 36 individuals, a heterozygous pattern $(+/-)$ was detected, indicating that both alleles are present (Fig. 3.1a). All studied specimens which were phenotypically identified as $S$. sciureus showed a homozygous absence of the integration, while phentotypically $S$. $b$. boliviensis showed a homozygous presence of the insertion. The specimens identified as $S$. $b$. peruviensis showed an insertion pattern with all possible combinations $(\mathrm{n}=26:+/-$, $\mathrm{n}=25:+/+, \mathrm{n}=2:-/-)$. Similar results were obtained for 24 individuals, which were identified phenotypically as hybrids ( $\mathrm{n}=10:+/-, \mathrm{n}=5:+/+, \mathrm{n}=9:-/-)$. 

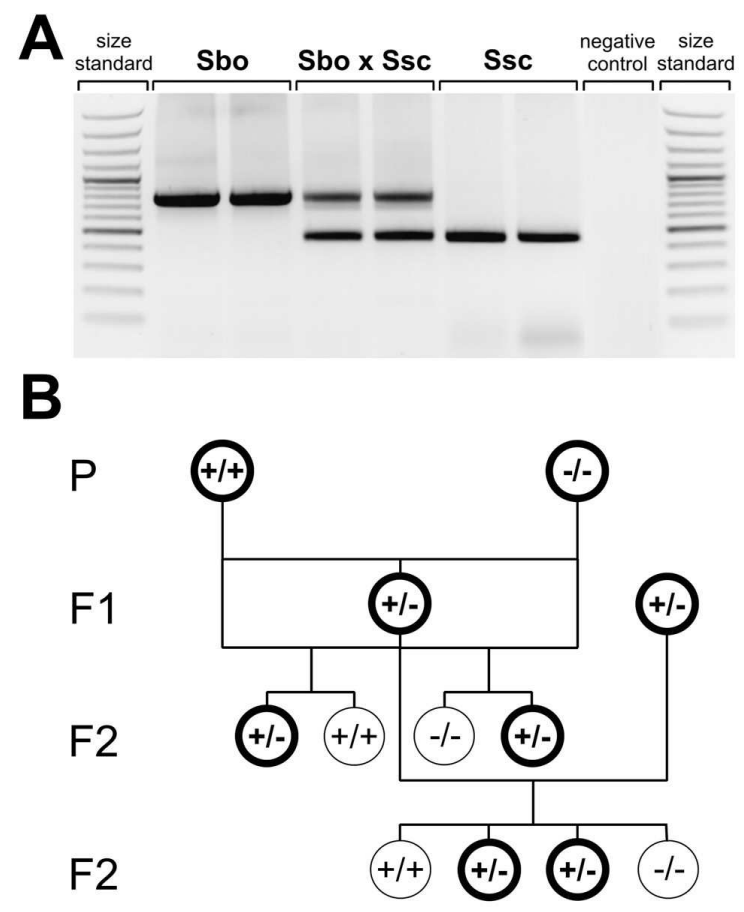

Figure 3.1: (A) Presence / absence analysis of the Alu integration as revealed by agarose gel electrophoresis. Pure S. boliviensis (Sbo) and S. sciureus (Ssc) show PCR product sizes of $\sim 750$ bp and $\sim 450 \mathrm{bp}$, respectively. Hybrids (Sbo $\mathrm{x}$ Ssc) possess both alleles. (B) Inheritance scheme of alleles with (+) and without (-) Alu integration. $F 1$ hybrids between pure $S$. sciureus (-/-) and $S$. boliviensis $(+/+)$ show a heterozygous (+/-) pattern, whereas in the F2 generation heterozygous $(+/-)$ as well as homozygous $(+/+;-/-)$ individuals are possible. Accordingly, the latter are falsely classified as pure breed animals (indicated by thin lines).

\subsection{Discussion}

The results of this study indicate that the analysed Alu insertion is specific for S. b. boliviensis, and hence, can be used to distinguish S. b. boliviensis from S. sciureus. However, contradicting results were obtained for S. b. peruviensis, since we found homozygous positive, homozygous negative and heterozygous insertion patterns. It is possible that our study specimens of $S$. b. peruviensis originated from a natural hybrid zone between S. sciureus and S. b. peruviensis, which has been reported from the margins of the Ucayali river in the Peruvian Amazonia (Silva et al., 1992). Moreover it cannot be excluded that S. b. peruviensis is the result of ancestral hybridization at all, as indicated by the fact that they are phenotypically intermediate between S. sciureus, and S. b. boliviensis, showing male head coloration as in the former and female head coloration as in the latter. However, recent molecular studies indicate a close affiliation of S. b. peruviensis and S. b. boliviensis (Boinski and Cropp, 1998; Cropp and Boinski, 2000). Therefore and due to the partial presence of the integration it seems likely that $S . b$. peruviensis from outside the hybrid zone may be homozygous positive.

Among the 24 phenotypically identified hybrids, ten individuals showed a heterozygous pattern indicating indeed that these animals are hybrids between S. b. boliviensis and S. sciureus. The other 14 animals show either homozygous presence or absence of the integration. With the herein presented marker, all F1 hybrids can be clearly defined, whereas in F2 hybrids only $50 \%$, those with 
heterozygous pattern, are traceable. F2 hybrids with either homozygous presence or absence patterns would be falsely classified as either pure S. b. boliviensis or S. sciureus (Fig. 3.1b)

Although with some drawbacks, we identified a potential molecular cladistic marker to distinguish between S. sciureus and S. b. boliviensis. The advantage of this marker is that the size of PCR products with and without integration differs by $\sim 300 \mathrm{bp}$, so that only agarose and no acrylamide gels or sequencing analyses are necessary. Accordingly, results can easily be determined and laboratory costs are relative low. However, since no other species than S. boliviensis and S. sciureus were analysed, it remains open which presence / absence pattern the other species will show. Another drawback of the marker is that only F1 hybrids and not those in further generations are traceable with significance. This will be overcome if more such markers will become available. Nevertheless, the presented marker provides a useful tool to easily distinguish pure breed S. sciureus and S. b. boliviensis, which may help improve captive breeding management.

\section{Acknowledgements}

We thank the staff of the zoos in Dresden, Gettorf, Madrid, Mannheim, Nuremberg, Romagne, Schwerin and the German Primate Center for providing samples of squirrel monkeys. We have adhered to the guidelines for the use of animals in research and the legal requirements of Germany. 


\section{Phylogenetic position of the langur genera Semnopithecus and Trachypithecus among Asian colobines, and affiliations of species groups}

Martin Osterholz ${ }^{1}$, Lutz Walter ${ }^{1,2}$, and Christian Roos*1,2,3

1Primate Genetics and ${ }^{2}$ Gene Bank of Primates, German Primate Center, Kellnerweg 4, 37077 Goettingen, Germany,

${ }^{3}$ IUCN/SSC Primate Specialist Group, Section Southeast Asia

*Corresponding author

BMC Evolutionary Biology 2008 Feb 25;8:58. 


\begin{abstract}
Background: The evolutionary history of the Asian colobines is less understood. Although monophyly of the odd-nosed monkeys was recently confirmed, the relationships among the langur genera Presbytis, Semnopithecus and Trachypithecus and their position among Asian colobines remained unclear. Moreover, in Trachypithecus various species groups are recognized, but their affiliations are still disputed. To address these issues, mitochondrial and $\mathrm{Y}$ chromosomal sequence data were phylogenetically related and combined with presence / absence analyses of retroposon integrations.

Results: The analysed $5 \mathrm{~kb}$ fragment of the mitochondrial genome allows no resolution of the phylogenetic relationships among langur genera, but five retroposon integrations were detected which link Trachypithecus and Semnopithecus. According to Y chromosomal data and a $573 \mathrm{bp}$ fragment of the mitochondrial cytochrome $b$ gene, a common origin of the species groups $T$. [cristatus], $T$. [obscurus] and T. [francoisi] and their reciprocal monophyly is supported, which is also underpinned by an orthologous retroposon insertion. T. [vetulus] clusters within Semnopithecus, which is confirmed by two retroposon integrations. Moreover, this species group is paraphyletic, with T. vetulus forming a clade with the Sri Lankan, and T. johnii with the South Indian form of S. entellus. Incongruence between gene trees was detected for T. [pileatus], in that $\mathrm{Y}$ chromosomal data link it with T. [cristatus], T. [obscurus] and T. [francoisi], whereas mitochondrial data affiliates it with the Semnopithecus clade.

Conclusions: Neither relationships among the three langur genera nor their position within Asian colobines can be settled with $5 \mathrm{~kb}$ mitochondrial sequence data, but retroposon integrations confirm at least a common origin of Semnopithecus and Trachypithecus. According to Y chromosomal and $573 \mathrm{bp}$ mitochondrial sequence data, T. [cristatus], T. [obscurus] and T. [francoisi] represent true members of the genus Trachypithecus, whereas T. [vetulus] clusters within Semnopithecus. Due to paraphyly of $T$. [vetulus] and polyphyly of Semnopithecus, a split of the genus into three species groups (S. entellus - North India, S. entellus - South India + T. johnii, S. entellus - Sri Lanka + T. vetulus) seems to be appropriate. T. [pileatus] posses an intermediate position between both genera, indicating that the species group might be the result of ancestral hybridization.
\end{abstract}

\title{
4.1 Background
}

The Old World monkeys are traditionally divided into the two subfamilies Cercopithecinae and Colobinae, which differ from each other by numerous morphological, behavioral and ecological characteristics (Napier, 1970; Szalay and Delson, 1979; Davies and Oates, 1994; Groves, 2001). While detailed information on the evolutionary history of cercopithecines (baboons, mangabeys, macaques and guenons) is at hand, knowledge on colobines is still scarce. Although some molecular studies (Rosenblum et al., 1997; Wang et al., 1997; Zhang and Ryder, 1998; Roos, 2003; Geissmann et al., 2004; Roos, 2004; Nadler et al., 2005; Whittaker et al., 2006; Roos et al., 2007) exist, they mainly focus on relationships within genera or species groups and not on the general phylogeny of the subfamily. Recently, a first mitochondrial phylogeny of colobine genera was established (Sterner et al., 2006), which confirmed some previous assumptions, but also led to confusions, calling for further research to definitively elucidate their evolutionary history.

Based on distribution and morphology, the colobines are traditionally divided into an African and an Asian clade (Davies and Oates, 1994; Groves, 2001), while Asian colobines are more diverse than their African relatives. Hence, the Asian forms are further split into the odd-nosed monkey (Pygathrix, Rhinopithecus, Nasalis, Simias) and langur (Presbytis, Trachypithecus, Semnopithecus) groups, 
which are both believed to be monophyletic. Accordingly, langurs were originally combined in the single genus Presbytis (Napier and Napier, 1967; Groves, 1970; Delson, 1975) or Semnopithecus (Reichenbach, 1862), but based on neonatal coloration and cranial morphology, they were split into the three genera Semnopithecus, Trachypithecus and Presbytis (Pocock, 1935), and a fourth genus (Kasi) was added (Hill, 1934). Alternatively, Semnopithecus was separated from Presbytis, with Trachypithecus forming a subgenus of the former (Brandon-Jones, 1984; Strasser and Delson, 1987; Brandon-Jones, 1995), but recent classifications use a subdivision of langurs into the three genera Presbytis, Trachypithecus and Semnopithecus (Weitzel et al., 1988; Groves, 1989; Davies and Oates, 1994; Groves, 2001; Brandon-Jones et al., 2004).
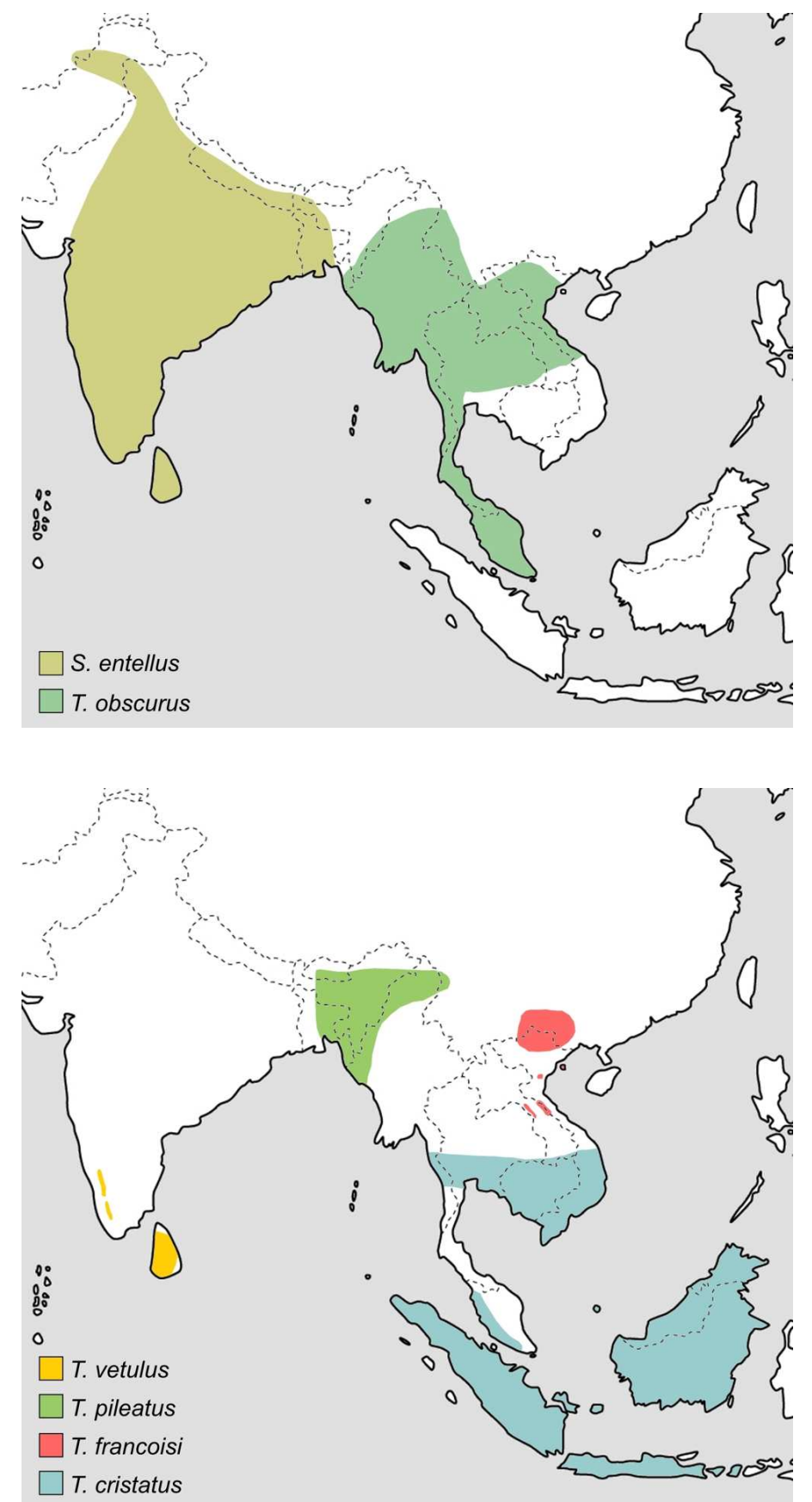

Figure 4.1: Distribution of the genus Semnopithecus and Trachypithecus [obscurus]. Genus affiliations and species groups after Groves (2001).
Figure 4.2: Distribution of the Trachypithecus species groups $T$. [vetulus], T. [pileatus], T. [francoisi] and T. [cristatus]. Genus affiliations and species groups after Groves (2001). 


\section{Phylogenetic position of the langur genera Semnopithecus and Trachypithecus among Asian colobines, and affiliations of species groups}

Within the different langur genera, several species are recognized, which are lumped into species groups due to similar fur coloration, behavior, ecology or distribution. With five species groups (T. [obscurus], T. [francoisi], T. [cristatus], T. [pileatus] and T. [vetulus]) (Groves, 2001), the genus Trachypithecus is the most diverse of all langurs and posses also the widest distribution, ranging from South India and Sri Lanka through mainland Southeast Asia to the Sundaland (Fig. 4.1, Fig. 4.2). Although all of them are morphologically similar, $T$. [vetulus] was sometimes separated in its own genus Kasi (Hill, 1934), and recent mitochondrial sequence data indicate a closer affiliation of $T$. [vetulus] and T. [pileatus] to Semnopithecus than to Trachypithecus (Zhang and Ryder, 1998; Geissmann et al., 2004). Accordingly, the two T. [vetulus] members were recognized as species of Semnopithecus (Brandon-Jones et al., 2004). In contrast to Trachypithecus, the genus Semnopithecus is restricted to the Indian subcontinent (Fig. 4.1) and traditionally regarded as monotypic with the only species S. entellus (Davies and Oates, 1994; Rowe, 1996), although recently several subspecies were elevated to species status (Groves, 2001). The third langur genus, Presbytis, includes several species, which occur solely in the Sundaland, but are not lumped into distinct species groups (Groves, 2001).

The phylogenetic relationships among the different Asian colobine genera are disputed. Although a common origin of the odd-nosed monkeys was recently confirmed (Sterner et al., 2006), evidence for monophyly of its putative sister clade, the langur group, is still lacking. Moreover, available data depict Trachypithecus and Presbytis as sister taxa to the exclusion of Semnopithecus (Sterner et al., 2006), which contradicts with traditional classifications, in which Trachypithecus and Semnopithecus are believed to form a clade to the exclusion of Presbytis (Brandon-Jones, 1984; Strasser and Delson, 1987; Brandon-Jones, 1995; Groves, 2001). These findings raise the question of what positions the langur genera occupy among Asian colobines and whether the langurs form a monophyletic clade in general. Moreover, the affiliations of different Trachypithecus species groups, especially T. [vetulus] and T. [pileatus], are disputed, and hence, led to different classifications. Currently, only few genetic data are available (Zhang and Ryder, 1998; Bigoni et al., 2003; Geissmann et al., 2004), so that further information from other markers is required to definitively establish their relationships.

To address all these issues, mitochondrial and $\mathrm{Y}$ chromosomal sequence data were phylogenetically related and combined with presence / absence analysis of retroposon integrations. This approach was used to simultaneously analyse paternal-, maternal- and biparental-inherited markers, which allow the detection of incongruence between different gene trees indicating possible hybridization or introgression events between different lineages (Evans et al., 2003; Tosi et al., 2002; Arnold and Meyer, 2006). To determine the phylogenetic position of the langur genera among Asian colobines, a $5 \mathrm{~kb}$ fragment of the mitochondrial genome was sequenced from eight colobine genera, and combined with presence / absence analysis of retroposon integrations. Retroposon insertion events are nearly homoplasy-free and precise excision of elements is highly unlikely (Shedlock and 
Okada, 2000; Batzer and Deininger, 2002). Accordingly, retroposon insertions are powerful informative markers, which were already successfully applied to elucidate phylogenetic relationships in various primate lineages (Schmitz et al., 2001; Roos et al., 2004; Ray et al., 2005; Schmitz et al., 2005; Xing et al., 2005). To study relationships among different langur species groups and their genus affiliations, a $573 \mathrm{bp}$ fragment of the mitochondrial cytochrome b gene and a 777 bp portion of the SRY (sex-determining region, $\mathrm{Y}$ chromosome) gene was sequenced from at least one representative per species group, and complemented with retroposon analysis.

\subsection{Results and Discussion}

\subsubsection{Genus level phylogeny}

To elucidate the phylogenetic relationships among the different langur genera and their position among Asian colobines, mitochondrial sequence studies were combined with presence / absence analysis of retroposon insertions.

The herein analysed $5 \mathrm{~kb}$ fragment of the mitochondrial genome was assembled from sequences derived from 1-2 kb long and partly overlapping PCR products, whereby no inconsistencies in overlapping sequence fragments were detected. As template material, mainly DNA extracted from feces was used, which minimizes the amplification of nuclear pseudogenes (Thalmann et al., 2004), and comparisons of the data with sequences already deposited at GenBank revealed only intra-species or -generic variation, indicating that no nuclear pseudogenes were amplified.

To determine the phylogenetic relationships among analysed genera, tree reconstructions were conducted with different algorithms, which all led to the same tree topology (Fig. 4.3). Moreover, most relationships are significantly supported and congruent with previous classifications (Napier, 1970; Szalay and Delson, 1979; Davies and Oates, 1994; Groves, 2001), indicating the reliability of the data set. In detail, the reconstructions confirm relationships among cercopithecine genera (Macaca, Papio, Chlorocebus), and reciprocal monophyly of cercopithecines and colobines, as well as of African and Asian colobines. Within the Asian clade, relationships are not resolved, although at least a common origin of the odd-nosed monkeys is depicted. Neither the assumed monophyly of the langur genera, nor the expected close affinity of Trachypithecus and Semnopithecus or the recently indicated sister grouping of Trachypithecus and Presbytis (Sterner et al., 2006) can be verified with significance. Moreover, all alternative tree topologies, in which the three langur genera were regarded as monophyletic, or variously recognized as sister clades to each other, to the odd-nosed monkey clade, or even as basal among Asian colobines, were not rejected ( $\mathrm{P}=0.097$ - 0.776). Accordingly, an 
unresolved polytomy among the three langur genera and the odd-nosed monkey clade may best reflect the relationships among Asian colobines based on the mitochondrial data.

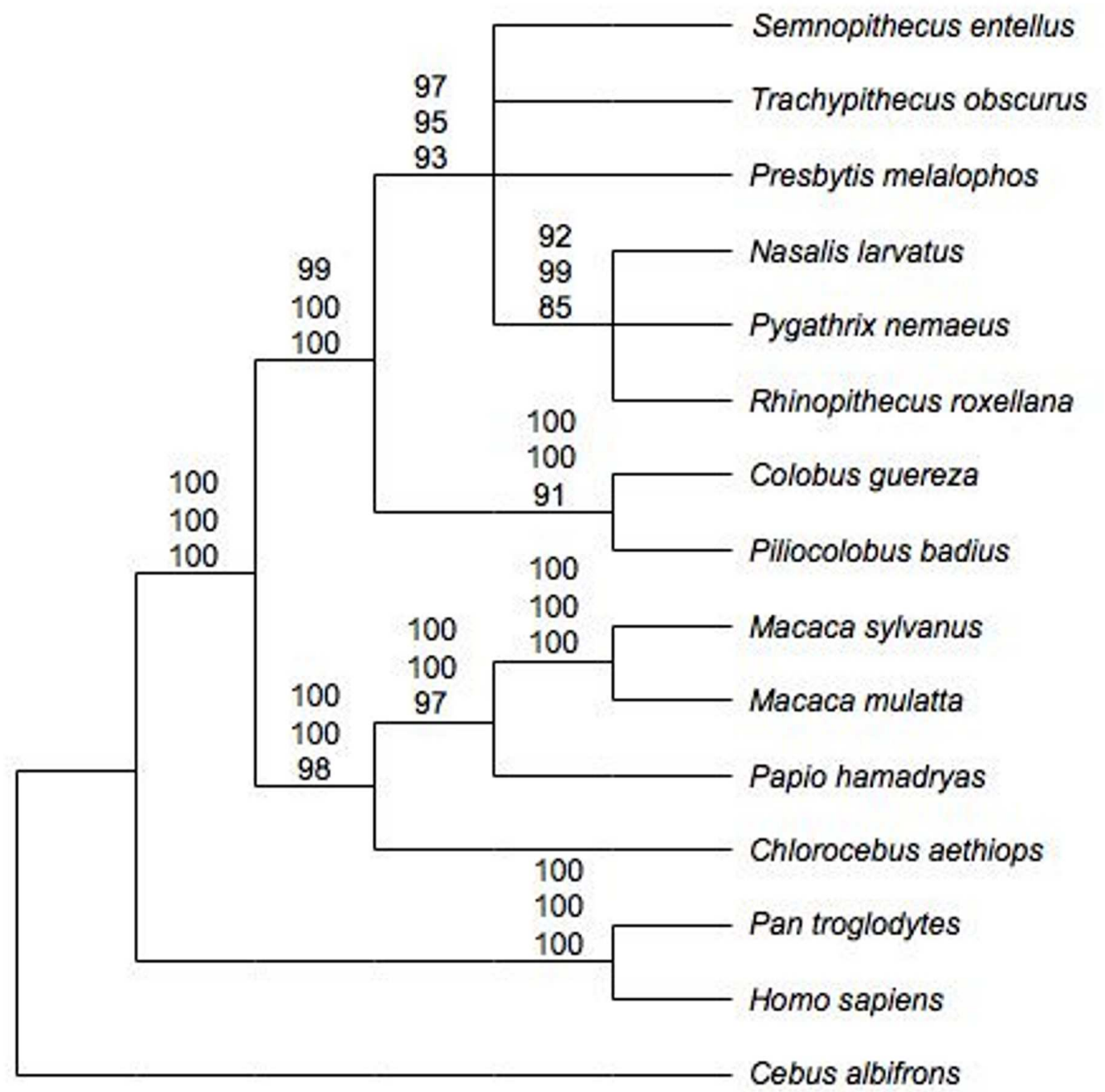

Figure 4.3: Phylogenetic relationships among colobines and related genera based on mitochondrial data. Numbers on nodes indicate support values $>80 \%$ (first: ML, second: NJ, third: MP).

Several retroposon insertions were detected, which provide insights into the branching pattern of colobines (Fig. 4.4). Together with seven already published integrations (Xing et al., 2005), now 14 loci confirm monophyly of the Asian colobines to the exclusion of the African genus Colobus. Among Asian colobines, one insertion indicates a common origin of the odd-nosed monkeys, which is verified by three further loci (Xing et al., 2005). Most prominent are the five retroposon integrations, which confirm a sister grouping of Trachypithecus and Semnopithecus. No loci were detected, which link Presbytis either with the other two langur genera or the odd-nosed monkeys. 


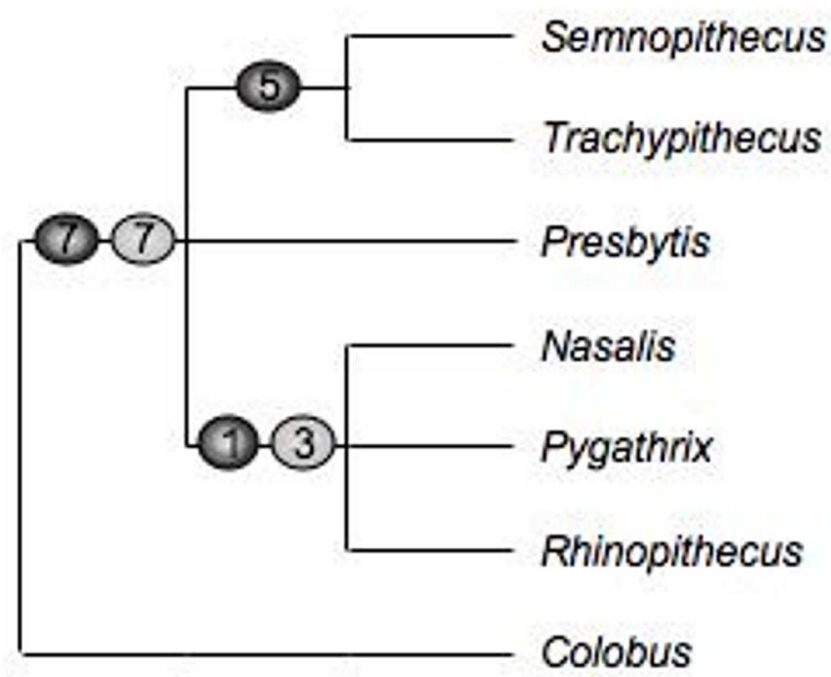

Figure 4.4: Phylogenetic relationships among Asian colobine genera based on retroposon integrations. Dark dots represent new generated data, whereas light dots refer to already published data (Xing et al., 2005). Numbers in dots indicate single integration events.

Although in general the mitochondrial data are suitable to elucidate relationships among the different genera, as indicated by the correct and significantly supported branching patterns among all other studied genera, the relationships among the langurs are unresolved, which is concordant with previous results (Sterner et al., 2006). In contrast, the presence / absence analysis of retroposon integrations provides evidence for a monophyletic odd-nosed monkey clade and a common origin of Trachypithecus and Semnopithecus, which is in agreement with morphological hypotheses (Napier, 1970; Brandon-Jones, 1984; Strasser and Delson, 1987; Davies and Oates, 1994; Brandon-Jones, 1995; Groves, 2001). Regardless which markers were used, the phylogenetic position of Presbytis among Asian colobines and accordingly the unity of the langurs remains unclear and needs further investigations.

\subsubsection{Species group phylogeny}

In order to settle affiliations among the different Trachypithecus species groups and their members, mitochondrial and $Y$ chromosomal sequence data were combined with information on retroposon integrations.

The mitochondrial phylogeny was established on the basis of $573 \mathrm{bp}$ long cytochrome $\mathrm{b}$ gene sequences, generated from most species recognized in the genus and its sister genus Semnopithecus. In all tree reconstructions, identical relationships were obtained, with most branches being significantly supported (Fig. 4.5a). Accordingly, the different species groups are divided into two major groups, with one including solely groups of the genus Trachypithecus, whereas the second one includes representatives of Trachypithecus and Semnopithecus. In the mixed clade, T. [vetulus] and T. [pileatus] members are lumped together with S. entellus. Whereby T. [pileatus] is monophyletic, the members of T. [vetulus] are paraphyletic, with T. vetulus forming a sister clade to S. entellus from Sri Lanka, and T. 
johnii with S. entellus from South India. Furthermore, a fourth lineage in the mixed clade was detected, which is represented by $S$. entellus from North India. In contrast, the three species groups (T. [obscurus], T. [francoisi], T. [cristatus]) in the clade comprising solely Trachypithecus groups are all monophyletic. Alternative relationships, in which $T$. [vetulus] is recognized as monophyletic, either $T$. [vetulus] or T. [pileatus] belongs to Trachypithecus or even both are members of Trachypithecus, were tested, but all of them were rejected $(\mathrm{P}<0.05)$.

With some exceptions, the $Y$ chromosomal data provide a similar picture (Fig. 4.5b), but due to the low number of polymorphic sites, support values are in general not as high as in the mitochondrial tree. According to the reconstructions, the species groups are divided into two major clades, with one comprising T. [obscurus], T. [cristatus], T. [francoisi] and T. [pileatus], and the other, T. [vetulus] and Semnopithecus. Relationships among the latter are not resolved. All alternative tree topologies, in which either T. [vetulus] belongs to Trachypithecus or T. pileatus groups with Semnopithecus, were rejected $(\mathrm{P}<0.05)$.

Retroposon insertions further deepened our knowledge on the species group relationships. Altogether, three informative integrations were analysed (Fig. 4.5c), with one occurring in $T$. [obscurus], T. [cristatus] and T. [francoisi], and the other two in T. [vetulus] and Semnopithecus. Interestingly, all three integrations are absent in T. pileatus.

With the exception of the varying position of $T$. [pileatus], the affiliations of the remaining species groups are congruent among different gene trees. Accordingly, all analysed markers relate T. [vetulus] with Semnopithecus, indicating that this species group is a real member of the genus Semnopithecus and not of Trachypithecus as assumed by morphological similarities (Groves, 2001). These similarities may be the results of adaptations to similar ecological conditions (Semnopithecus is semi-terrestrial and lives in deciduous forest, whereas Trachypithecus including T. [vetulus] is arboreal and occurs in wet evergreen forests). Although the Y chromosomal data allow no resolution within the Semnopithecus - T. [vetulus] clade, the mitochondrial data indicate paraphyly of the two T. [vetulus] species, with $T$. vetulus clustering with S. entellus from Sri Lanka and T. johnii with S. entellus from South India, which is concordant with their geographical distribution. These findings indicate paraphyly of S. entellus, whereby North Indian representatives form a further distinct lineage. Accordingly, the langurs of the Indian subcontinent should be split into three species groups, with one occurring solely on Sri Lanka, one in Southern India and a third one in Northern India, whereas the Gondavari river seems to be barrier between the latter two. 

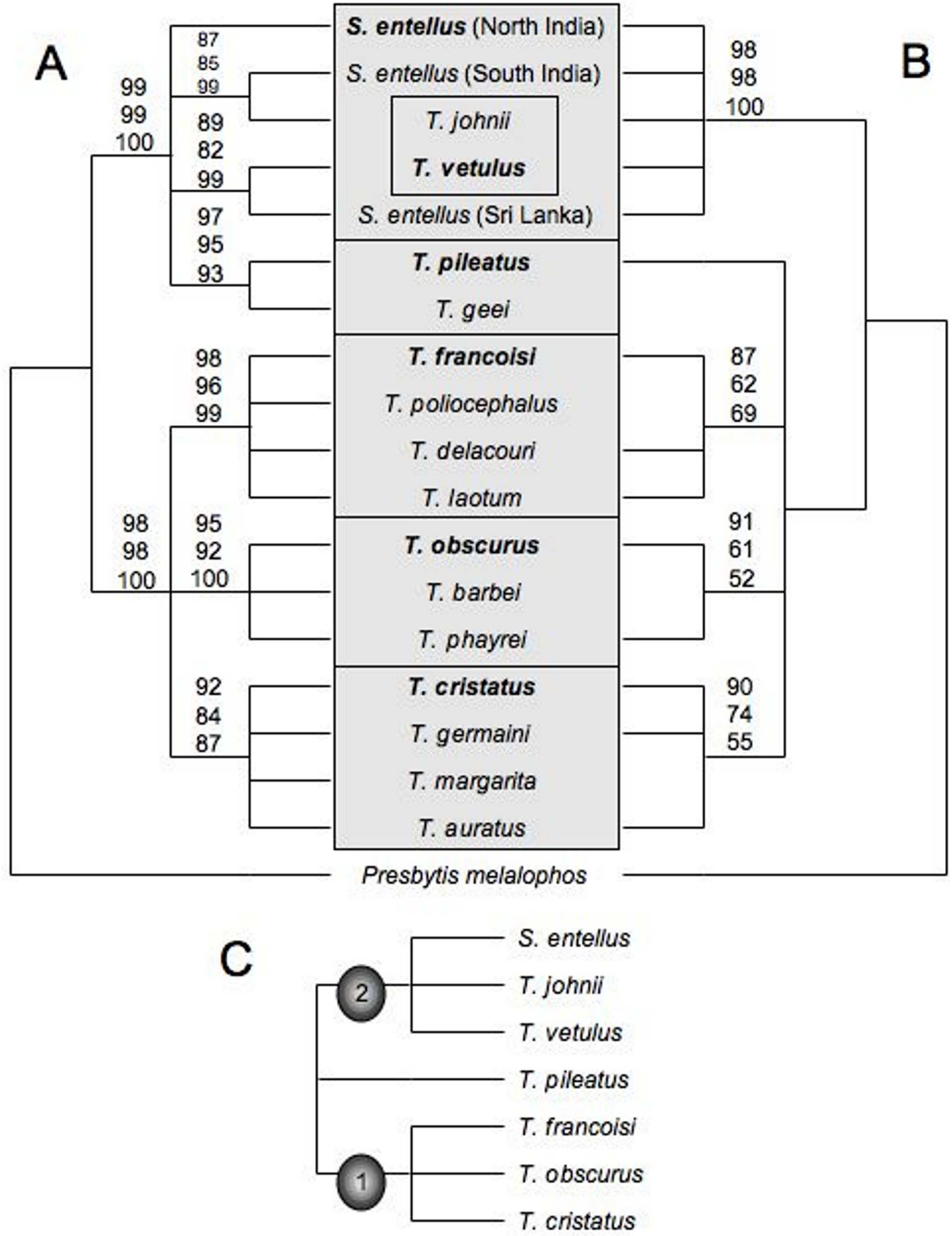

Figure 4.5: Phylogenetic relationships among Semnopithecus and Trachypithecus species groups based on a) mitochondrial data, b) Y chromosomal data, and c) retroposon integrations. Numbers on nodes indicate support values (first: ML, second: NJ, third: MP), and boxed species belong to a species group, with species in bold giving the name of the group. 
Monophyly of each of T. [obscurus], T. [cristatus] and T. [francoisi] and their close affiliation is depicted in all gene trees, so that all of them can be regarded as true members of Trachypithecus. These findings confirm previous molecular studies (Roos, 2003; Geissmann et al., 2004; Roos, 2004; Nadler et al., 2005; Roos et al., 2007) and are in general agreement with recent classifications (Groves, 2001; Brandon-Jones et al., 2004).

The only discrepancies between different gene trees were obtained for T. [pileatus]. Whereas the mitochondrial data link the species group with Semnopithecus and T. [vetulus], the Y chromosomal data affiliates it with $T$. [obscurus], T. [cristatus] and T. [francoisi]. These findings might be explained by incomplete lineage sorting of ancestral mitochondrial or $Y$ chromosomal haplotypes. Accordingly, the ancestor of Trachypithecus, Semnopithecus and T. [pileatus] carried multiple DNA lineages with one lineage being randomly fixed in two taxa, but not in the third. Alternatively, the varying position of $T$. [pileatus] in different gene trees might be explained by past hybridization between Semnopithecus and Trachypithecus. As depicted by the three retroposon insertions, this putative hybridization event would have occurred between ancestral forms of Semnopithecus and Trachypithecus, before both genera diverged into distinct species groups. The hybridization hypothesis is also supported by some intermediate morphological characteristics (Groves, 2001) and the distribution of T. [pileatus], which is sandwiched between those of Semnopithecus and other Trachypithecus species groups (Fig. 4.1, Fig. 4.2).

\subsection{Conclusions}

The present study provides detailed insights into the evolutionary history of Asian colobines and underpins the tremendous power of retroposon integrations as cladistic markers. Although mitochondrial data proved to be useful to elucidate and confirm several relationships among studied taxa, the data set failed to resolve the affiliations among the langur genera and to settle their position among Asian colobines. In contrast, retroposon insertions provided clear evidence for a sister grouping of Semnopithecus and Trachypithecus, but no integrations were detected, which link Presbytis either with the other two langur genera or with the odd-nosed monkeys, so that further research is required to solve this issue. Moreover, to definitively explain the evolutionary history of colobines, further molecular markers should be analysed, especially regarding possible discrepancies among gene trees due to hybridization or introgression, as such events are important speciation mechanisms in primates (Arnold and Meyer, 2006), and as it was possibly detected in the present study in the case of T. [pileatus]. Although further investigations are necessary to fully understand the evolution of colobines, the present study provides sufficient data to revise the current classification of the genera Trachypithecus and Semnopithecus (Table 4.1). Accordingly, within Trachypithecus, three reciprocal monophyletic species groups (T. [obscurus], T. [cristatus] and T. [francoisi]) should be recognized, whereas T. [vetulus] should be included in Semnopithecus. 
Table 4.1: Proposed classification of Semnopithecus and Trachypithecus species based on the herein presented data.

\begin{tabular}{|c|c|c|}
\hline Species group & Semnopithecus & Trachypithecus \\
\hline S. entellus ${ }^{*}$ (North India) & S. entellus ${ }^{\star}$ & \\
\hline S. entellus ${ }^{*}$ (South India) & S. entellus & \\
\hline S. entellus ${ }^{*}$ (Sri Lanka) & S. entellus* & \\
\hline & S. vetulus & \\
\hline T. pileatus ${ }^{\star *}$ & $\begin{array}{l}\text { T. pileatus } \\
\text { T. geei }\end{array}$ & \\
\hline T. francoisi & & $\begin{array}{l}\text { T. francoisi } \\
\text { T. poliocephalus } \\
\text { T. delacouri } \\
\text { T. laotum }\end{array}$ \\
\hline T. obscurus & & $\begin{array}{l}\text { T. obscurus } \\
\text { T. barbei } \\
\text { T. phayrei }\end{array}$ \\
\hline T. cristatus & & $\begin{array}{l}\text { T. cristatus } \\
\text { T. germaini } \\
\text { T. margarita } \\
\text { T. auratus }\end{array}$ \\
\hline
\end{tabular}

* species / subspecies designation has to be assessed.

** provisionally classified as member of Trachypithecus.

Moreover, due to their paraphyletic origin, $T$. vetulus and $T$. johnii should not be lumped into a single species group, and S. entellus should not be regarded as monotypic. A classification into three species groups with one occurring in Northern India, one in Southern India and one on Sri Lanka may best reflect the evolutionary relationships among the langurs of the Indian subcontinent. However, this arrangement is tentative, because further research is required to confirm their distinctiveness not only on mitochondrial, but also on nuclear DNA level. T. [pileatus] might be the result of an ancient hybridization event between Semnopithecus and Trachypithecus, and hence, its classification is difficult. While a separation in its own distinct genus may be appropriate, we provisionally accept its traditional recognition as member of Trachypithecus.

\subsection{Methods}

\subsubsection{Sample collection, DNA extraction and preventing contaminations}

The species analysed in this study are presented in Table 4.2. All study specimens were identified by fur coloration and other external characteristics. Hanuman langur (S. entellus) samples were collected only from founder animals, of which the area of capture was at least roughly known. Total genomic DNA was extracted from blood, tissue or feces using the DNeasy or Stool Mini Kits from 
Qiagen. When hair follicle cells were used, 1-3 hairs were directly implemented into the PCR reaction after they were washed with sterile water and $95 \%$ ethanol. To prevent contaminations, sample collection and laboratory procedures followed described standard protocols (Taberlet et al., 1999; Goossens et al., 2000; Nsubuga et al., 2004; Karanth et al., 2005). In detail, all fecal and hair samples were collected with gloves and stored in sterile tubes or plastic bags before further processing. DNA extraction, PCR, gel extraction and sequencing was performed in separate laboratories and repeated after several months, while always only one individual per species was tested. Moreover, from most specimens two different sample types were available, which both were used as template material. Sequences from independent analyses were identical. Finally, all PCR reactions were performed with negative (distilled water) controls.

Table 4.2: Species analysed, their origin, material type and GenBank accession numbers.

\begin{tabular}{|c|c|c|c|c|c|}
\hline Species & Origin & $\begin{array}{c}\text { Material } \\
\text { type }\end{array}$ & $\begin{array}{c}\text { mtDNA } \\
(5 \mathrm{~kb})\end{array}$ & $\begin{array}{l}\text { mtDNA } \\
\text { (573 bp) }\end{array}$ & $\begin{array}{c}\text { SRY } \\
\text { (777 bp) }\end{array}$ \\
\hline Cebus albifrons & GenBank & sequence & NC_002763 & - & - \\
\hline Homo sapiens & GenBank & sequence & X933334 & - & - \\
\hline Pan troglodytes & GenBank & sequence & NC_001643 & - & - \\
\hline Chlorocebus aethiops & GenBank & sequence & NC_007009 & - & - \\
\hline Papio hamadryas & GenBank & sequence & NC_001992 & - & - \\
\hline Macaca mulatta & GenBank & sequence & NC_005943 & - & - \\
\hline M. sylvanus & GenBank & sequence & NC_002764 & - & - \\
\hline Colobus guereza & Cologne Zoo & tissue, feces & EŪ004483 & - & - \\
\hline Piliocolobus badius & MPI Leipzig & tissue, feces & EU004482 & - & - \\
\hline Pygathrix nemaeus & Cologne Zoo & tissue, feces & EU004481 & - & - \\
\hline Rhinopithecus avunculus & EPRC & tissue & EU004480 & - & - \\
\hline Nasalis larvatus & Wilhelma Stuttgart & blood & EU004476 & - & - \\
\hline Presbytis melalophos & Howletts Zoo & tissue, feces & EU004479 & part of $5 \mathrm{~kb}$ & EU004456 \\
\hline $\begin{array}{l}\text { Semnopithecus entellus } \\
\text { (North India) }\end{array}$ & Dresden Zoo & blood, feces & EU004478 & part of $5 \mathrm{~kb}$ & EU004457 \\
\hline S. entellus (South India) & Hannover Zoo & blood, feces & - & EU004471 & EU004458 \\
\hline S. entellus (Sri Lanka) & Krefeld Zoo & hairs & - & AY519452 & EU004459 \\
\hline Trachypithecus vetulus & Bristol Zoo & blood, feces & - & AY519454 & EU004461 \\
\hline T. johnii & Erfurt Zoo & hairs, feces & - & AY519453 & EU004460 \\
\hline T. pileatus & ZMB & tissue & - & EU004472 & EU004462 \\
\hline T. geei & GenBank & sequence & - & AF294618 & - \\
\hline T. obscurus & Wuppertal Zoo & blood, feces & EU004477 & part of $5 \mathrm{~kb}$ & EU004463 \\
\hline T. phayrei & $\mathrm{ZMB}$ & tissue & - & AY519460 & EU004464 \\
\hline T. barbei & GenBank & sequence & - & AY519462 & - \\
\hline T. auratus & Wilhelma Stuttgart & blood & - & AY519455 & EU004468 \\
\hline T. cristatus & Singapore Zoo & blood, feces & - & EF465128 & EU004470 \\
\hline T. germaini & ACCB & feces & - & AY519457 & EU004469 \\
\hline T. margarita & GenBank & sequence & - & EF465147 & - \\
\hline T. francoisi & Bristol Zoo & hairs, feces & - & AY519458 & EU004467 \\
\hline T. poliocephalus & EPRC & feces & - & EU004473 & - \\
\hline T. delacouri & EPRC & blood, feces & - & EU004474 & EU004465 \\
\hline T. laotum & EPRC & blood, feces & - & EU004475 & EU004466 \\
\hline
\end{tabular}

Abbreviations: ACCB - Angkor Center for Conservation of Biodiversity, Cambodia; EPRC Endangered Primate Rescue Center, Vietnam; MPI Leipzig - Max-Planck-Institute for Evolutionary Anthropology, Leipzig, Germany; ZMB - Zoologisches Museum Berlin, Germany 


\subsubsection{Mitochondrial sequence analysis}

To determine the phylogenetic position of langur genera among Asian colobines, a $\sim 5 \mathrm{~kb}$ fragment of the mitochondrial genome was sequenced from all colobine genera with the exception of Simias and Procolobus. This region spans the cytochrome b gene, the control region, the $12 S$ rDNA and $16 S$ rDNA, and the intermediate tRNAs. To exclude contaminations of the dataset with nuclear pseudogenes, mainly DNA extracted from feces was used as template material, and $\sim 1-2 \mathrm{~kb}$ long and 200-400 bp overlapping fragments were amplified. PCR products were generated via hot-start technique using a set of 24 primers (Table 4.3) and PCR conditions comprising a pre-denaturation step at $94^{\circ} \mathrm{C}$ for $2 \mathrm{~min}$, followed by 40 cycles at $94^{\circ} \mathrm{C}$ for $1 \mathrm{~min}, 60^{\circ} \mathrm{C}$ for $1 \mathrm{~min}$ and $72^{\circ} \mathrm{C}$ for $1-2 \mathrm{~min}$, and a final extension step at $72^{\circ} \mathrm{C}$ for $5 \mathrm{~min}$. The results of the PCR amplifications were checked on agarose gels. PCR products were cleaned with the Qiagen PCR Purification Kit and subsequently sequenced on an ABI 3100-Avant sequencer using the BigDye Terminator Cycle Sequencing Kit (Applied Biosystems).

Table 4.3: Primers for amplifying and sequencing the $5 \mathrm{~kb}$ mitochondrial fragment.

\begin{tabular}{|c|c|}
\hline Primer* & Sequence (5'-3') \\
\hline L14182 & AAACCATCGTTGTATTTCAACTA \\
\hline L14621 & GAGGACAAATATCATTTTGAGG \\
\hline $\mathrm{H} 14886$ & GTAGGGGTGGAAGGGAATTT \\
\hline L15139 & CACAAATCCAAACAACAAAGCA \\
\hline H15246 & ACCGGTTGGCTTCCAATTCA \\
\hline L15322 & CTCCTCAAATGAACTTGCCC \\
\hline $\mathrm{H} 15556$ & GCAGTAATGCACGAATTACATA \\
\hline L15873 & CCATCCTCCGTGAAATCAATA \\
\hline $\mathrm{H} 16117$ & TGCAGACCAGAGATAAAAGATA \\
\hline L16248 & GGTGTTATTTAATCCATGCTTG \\
\hline $\mathrm{H} 16402$ & TGTTTTTGGGGTTTGGCAAAG \\
\hline L104 & TTAGCAAGATTACACATGCAAG \\
\hline $\mathrm{H} 284$ & CATAGCTTAGTTAAACTTTCGTT \\
\hline L501 & CACTATGCTTAGCCCTAAACT \\
\hline $\mathrm{H} 572$ & AAGCTGTTGCTTGTAGTGTTC \\
\hline L827 & AAGAGTCCAAGGAGGATTTAG \\
\hline H999 & CCAGTACACTTACCATGTTAC \\
\hline L1331 & ACGAGCTACCCAAAAACAGC \\
\hline $\mathrm{H} 1535$ & TAAAGAGCTGTCCCTCTTTAG \\
\hline L1608 & TTAAGAAAGCGTTCAGCTCAA \\
\hline H1883 & TCСТTTTAСТTTTTTTAАССТTТС \\
\hline L2090 & CCTGACCGTGCAAAGGTAG \\
\hline $\mathrm{H} 2342$ & TCCGAGGTCACCCCAACC \\
\hline $\mathrm{H} 2670$ & ATTACCGGGCTCTGCCATC \\
\hline
\end{tabular}

* Numbers refer to positions in the Macaca sylvanus mitochondrial genome, and $\mathrm{H}$ and L refer to the heavy and light strands, respectively. 
To obtain a comprehensive overview on the phylogeny of colobines, the dataset was expanded with further sequences from related taxa deposited at GenBank (Table 4.2). Accordingly, the final dataset comprised 15 taxa, including eight colobines, four cercopithecines, two hominoides and a New World monkey, which was used as outgroup taxon. Sequences were aligned with ClustalW (Thompson et al., 1994) and subsequently checked by eye. Gaps and poorly aligned positions were removed with the G-blocks software (Castresana, 2000), which reduced the final dataset to $4336 \mathrm{bp}$. Based on this alignment, phylogenetic trees were constructed with the maximum-parsimony (MP), neighbor-joining (NJ) and maximum-likelihood (ML) algorithms as implemented in PAUP 4.0b10 (Swofford, 2002) and TREEPUZZLE 5.0 (Strimmer and von Haeseler, 1996). For MP analyses, all characters were treated as unordered and equally weighted throughout. A heuristic search was performed with the tree-bisection-reconnection (TBR) algorithm with random addition of sequences. The maximum number of trees was set to 100 . NJ and ML trees were constructed with the GTR + I (= $0.2526)+\Gamma(=0.4816)$ model of sequence evolution as it was selected as best-fitting model under the Akaike information criterion with MODELTEST 3.06 (Posada and Crandall, 1998). Relative support of internal nodes was performed by bootstrap analyses with 1,000 replications (MP, NJ), or by the quartet puzzling support values on the basis of 1,000 puzzling steps (ML). Finally, to evaluate the reliability of the depicted phylogenetic position of the langur genera among Asian colobines, alternative tree topologies were evaluated with the Kishino-Hasegawa (Kishino and Hasegawa, 1989) and Shimodaira-Hasegawa (Shimodaira and Hasegawa, 1999) tests in PAUP, both performed with full optimization and 1,000 bootstrap replications. Therefore, all three langur genera were regarded as monophyletic or variously recognized as sister clade to each other, to the odd-nosed monkey clade or even as sister clade to all other Asian colobines.

To determine phylogenetic affiliations of species groups, a $573 \mathrm{bp}$ long fragment of the mitochondrial cytochrome $b$ gene was analysed from all species of the different groups (Table 4.2). The generation of sequences followed laboratory methods as described (Geissmann et al., 2004; Nadler et al., 2005; Roos et al., 2007). The final alignment, which was easily generated by eye due to the absence of insertions or deletions, comprised 19 individuals including the outgroup taxon Presbytis melalophos. Phylogenetic trees were constructed as described above. As best-fitting model, MODELTEST selected the TIM + I (= 0.5977) $+\Gamma$ (=2.3137) model, which was applied for NJ and ML reconstructions. As for the $5 \mathrm{~kb}$ fragment, several alternative tree topologies, in which $T$. [vetulus] is recognized as monophyletic, either T. [vetulus] or T. [pileatus] belongs to Trachypithecus, or even both are members of Trachypithecus, were tested. 


\subsubsection{Y chromosomal sequence analysis}

The SRY gene was selected as it represents a single-copy gene and as it is proved to be informative in reconstructing the $\mathrm{Y}$ chromosomal evolutionary history of macaques (Tosi et al., 2000). PCR conditions and primers were applied as described (Tosi et al., 2000). To amplify the SRY gene from fecal material, two overlapping fragments were generated with published primers (Tosi et al., 2000) and the newly generated internal primers 5'-TGGGCGGAGTTGAGAGGGGT-3' and 5'TAGCGGTCCCGTTGCTGCGG-3'. The final alignment of 777 bp comprised 15 taxa representing all species groups of the genera Semnopithecus and Trachypithecus as well as Presbytis melalophos, which was used as outgroup. To reconstruct $\mathrm{NJ}$ and ML trees, the K80 model of sequence evolution, determined with MODELTEST, was used. MP trees were generated as described above. The reliability of the depicted position of T. [vetulus] and T. [pileatus] was tested in PAUP by using alternative tree topologies, in which either T. [vetulus] belongs to Trachypithecus or T. pileatus groups with Semnopithecus.

\subsubsection{Retroposon analysis}

Due to their high copy number and relative small size $(\sim 300 \mathrm{bp})$, the primate specific Alu elements were selected as cladistic markers. The presence or absence of Alu elements in different colobines at specific loci was tested via PCR using primers occupying the flanking region of the insertion site. Details on analysed loci, primers and studied species are listed in Table 4.4.

To detect new loci, a subtractive hybridization approach (Mamedov et al., 2005) was performed with some modifications. As tracer and driver, different colobine genera were selected. Genomic DNA of tracer and driver was digested with RSAI (Fermentas), and subsequently, the adapters AdapA1/AdapAA1 (5'- TGTAGCGTGAAGACGACAGAAAGGGCGTGGTGCGGAGGGCGGT-3' / 5'- ACCGCCCTCCG-3') and AdapA2/AdapAA2 (5'-TGTAGCGTGAAGACCTGTCTTAGGGCGTGG TGGCCAGGGCCGT-3' / 5'- ACGGCCCTGGC-3') were ligated to the tracer fragments. Each of $~ 15 \mathrm{ng}$ tracerA1 and tracerA2 were hybridized with $\sim 1,500 \mathrm{ng}$ driver for $20 \mathrm{~h}$ at $60^{\circ} \mathrm{C} .2 \mu \mathrm{l}$ of the hybridization result was used as template to amplify solely tracer fragments using the adapter primers A1 (5'TGTAGCGTGAAGACGACAGAA-3') and A2 (5'-TGTAGCGTGAAGACCTGTCTT-3'). The PCR program consisted of a pre-extension step at $72^{\circ} \mathrm{C}$ for $6 \mathrm{~min}$ to fill in adaptor ends, followed by 25 cycles, each with a denaturation step at $95^{\circ} \mathrm{C}$ for $1 \mathrm{~min}$, annealing at $60^{\circ} \mathrm{C}$ for $1 \mathrm{~min}$ and extension at $72^{\circ} \mathrm{C}$ for 2 min. To enrich fragments with Alu insertions, a semi-nested PCR was added using either primer A1 or A2 and the Alu-specific AluY (5'-GGAGAATGGCGTGAACCCGGGA-3') oligonueclotide. The PCR products were separated on agarose gels and fragments over $500 \mathrm{bp}$ were excised from the gel. After purification, the fragments were cloned into the pGEMTeasy vector (Promega) and transformed into electro-competent TOP10 cells (Invitrogen). 
Table 4.4: Presence / absence analysis of retroposon insertions.

\begin{tabular}{|c|c|c|c|}
\hline Locus & Primer (5'-3') & Presence & Absence \\
\hline Asia1 & $\begin{array}{l}\text { AAGAATCCCAGGGAAGAACACT } \\
\text { TTGCTGGCAAAGTGACTCCT }\end{array}$ & $\begin{array}{l}\text { SENT, TOBS, PMEL, } \\
\text { PNEM, NLAR }\end{array}$ & CGUE \\
\hline Asia2 & $\begin{array}{l}\text { CCTGCCACTTCTGTCCATCT } \\
\text { AGAACAAACACCAAGACAACAGC }\end{array}$ & $\begin{array}{l}\text { SENT, TOBS, PMEL, } \\
\text { PNEM, NLAR }\end{array}$ & CGUE \\
\hline Asia3 & $\begin{array}{l}\text { GCTTTGCCACATAAAGAGCTG } \\
\text { GGTTAGGTGCAAATGGGAAAC }\end{array}$ & $\begin{array}{l}\text { SENT, TOBS, PMEL, } \\
\text { PNEM, NLAR }\end{array}$ & CGUE \\
\hline Asia4 & $\begin{array}{l}\text { TCAATCTTCCAGGGAAAATAAAG } \\
\text { GAATATTAGTTGAAATATTTAGGC }\end{array}$ & $\begin{array}{l}\text { SENT, TOBS, PMEL, } \\
\text { PNEM, NLAR }\end{array}$ & CGUE \\
\hline Asia5 & $\begin{array}{l}\text { GACCATGGTAAGACAAATGTG } \\
\text { GACTCAGGCTTAATTTTAAGTC }\end{array}$ & $\begin{array}{l}\text { SENT, TOBS, PMEL, } \\
\text { PNEM, NLAR }\end{array}$ & CGUE \\
\hline Asia6 & $\begin{array}{l}\text { CACCAAGCACAACTGTGAGG, } \\
\text { TCTGCCATAGCCATCAGTCA }\end{array}$ & $\begin{array}{l}\text { TOBS, PMEL, PNEM, } \\
\text { NLAR }\end{array}$ & CGUE \\
\hline Asia7 & $\begin{array}{l}\text { CTCTTGGTTGGGGTGAAGC } \\
\text { GATGGTTGAACAGTGAGACTTGA }\end{array}$ & $\begin{array}{l}\text { SENT, TOBS, PMEL, } \\
\text { PNEM, NLAR }\end{array}$ & CGUE \\
\hline ST1 & $\begin{array}{l}\text { TGATTAAAGTCAGATGAACACC } \\
\text { GTGTAATGGGATGAAGAACAC }\end{array}$ & $\begin{array}{l}\text { SENT, TVET, TDEL, } \\
\text { TOBS, TAUR }\end{array}$ & PMEL, PNEM, NLAR \\
\hline ST2 & $\begin{array}{l}\text { ATACATAGCATTGACTTAACTCT } \\
\text { GATCCTGAGCCCACTATTCT }\end{array}$ & SENT, TOBS & PMEL, PNEM, NLAR \\
\hline ST3 & $\begin{array}{l}\text { ACATCAGTGACATCAAATAAGG } \\
\text { GAGGAAAAGATACTTTCTCATG }\end{array}$ & SENT, TOBS & PMEL, PNEM, NLAR \\
\hline ST4 & $\begin{array}{l}\text { GGATTGAGAGCAATTTTAAAAGGA } \\
\text { GTTCACTCCCAAATCATACTTC }\end{array}$ & SENT, TOBS & $\begin{array}{l}\text { PMEL, PNEM, NLAR, } \\
\text { CGUE }\end{array}$ \\
\hline ST5 & $\begin{array}{l}\text { TGTAGCCAGGGAAGCCTCT } \\
\text { TGGGATTTCTAATACTATGCCTTTG }\end{array}$ & SENT, TOBS & $\begin{array}{l}\text { PMEL, PNEM, NLAR, } \\
\text { CGUE }\end{array}$ \\
\hline odd1 & $\begin{array}{l}\text { AGAAAGTCCCTCCCCAACAC } \\
\text { AAGTTGGCAAAGTGGATTGC }\end{array}$ & PNEM, NLAR, RAVU & $\begin{array}{l}\text { SENT, TOBS, PMEL, } \\
\text { CGUE }\end{array}$ \\
\hline T1 & $\begin{array}{l}\text { GAAGATTAATACTAGAAGAATCC } \\
\text { TTGAACTTTGATCCATGGTGC }\end{array}$ & TDEL, TOBS, TAUR & $\begin{array}{l}\text { TPIL, SENT, TVET, } \\
\text { PMEL, PNEM }\end{array}$ \\
\hline S1 & $\begin{array}{l}\text { CAAATTGTGGCTCCTTCAGTTA } \\
\text { GGCAATGTACAGCTAACTCTGCT }\end{array}$ & SENT, TVET, TJOH & $\begin{array}{l}\text { TPIL, TDEL, TOBS, } \\
\text { TAUR, PMEL, NLAR }\end{array}$ \\
\hline S2 & $\begin{array}{l}\text { CCCATGTGCCTTGGTTTAG } \\
\text { GGAAGAAAGTTTGGAATGTGTG }\end{array}$ & SENT, TVET, TJOH & $\begin{array}{l}\text { TPIL, TDEL, TOBS, } \\
\text { TAUR, PMEL, NLAR }\end{array}$ \\
\hline
\end{tabular}

Abbreviations: CGUE - Colobus guereza, NLAR - Nasalis larvatus, PMEL - Presbytis melalophos, PNEM Pygathrix nemaeus, RAVU - Rhinopithecus avunculus, SENT - Semnopithecus entellus, TAUR Trachypithecus auratus, TDEL - T. delacouri, TJOH - T. johnii, TOBS - T. obscurus, TPIL - T. pileatus, TVET - T. vetulus.

Bacterial clones were collected in 96-well microtiter plates and re-screened via PCR with the primers A1 or A2 and AluY. Positive clones were sequenced and analysed with REPEATMASKER and BLAST as implemented in NCBI and EMBL. Based on the generated alignments, locus-specific primers were constructed, with the forward primer occupying a region 5'-end upstream of the insertion site, which is conserved among the colobine and human or chimp sequences. Due to the absence of the 3 '-end downstream sequence of the tested colobine species, reverse primers were constructed solely on the basis of human or chimp sequences. Subsequently, the presence or absence of respective Alu insertions in different colobine species was tested via PCR. The orthology of insertions was confirmed by sequencing of at least one species per genus or species group. Sequences were deposited at GenBank and are available under the accession numbers EU004484-EU004537 and EU006662-EU006692. 


\section{Acknowledgements}

We are grateful to the staff of the zoos in Cologne, Dresden, Hannover, Krefeld, Erfurt, Stuttgart, Wuppertal, Howletts, Bristol and Singapore, and to Manfred Ade (Naturhistorisches Museum Berlin, Germany), Linda Vigilant (Max-Planck-Institute of Evolutionary Anthropology, Leipzig, Germany), Kai-Olaf Krüger (Angkor Centre for Conservation of Biodiversity, Cambodia) and Tilo Nadler (Endangered Primate Rescue Center, Vietnam) for samples. Finally, we thank Christiane Schwarz for her excellent technical assistance in the laboratory. The work was financially supported by the German Primate Center (DPZ). 


\title{
5 Mitochondrial versus nuclear DNA: The complex evolutionary history of colobine monkeys
}

\author{
Martin Osterholz ${ }^{* 1}$, Dietmar Zinner2, Christiane Schwarz ${ }^{1}$, Stephen Nash³, Lutz Walter ${ }^{1,3}$, \\ Christian $\operatorname{Roos}^{* 1,4,5}$
}

\begin{abstract}
${ }^{1}$ Department of Primate Genetics, ${ }^{2}$ Department of Cognitive Ethology, ${ }^{4}$ Gene Bank of Primates, German Primate Center, Kellnerweg 4, 37077 Goettingen, Germany, 3Department of Anatomical Sciences, HSC, SUNY, Stony Brook, NY, 5IUCN/SSC Primate Specialist Group, Section Southeast Asia
\end{abstract}

${ }^{*}$ Corresponding authors

to be submitted in a modified form to Current Biology 


\begin{abstract}
Background: From a genetic perspective, colobine monkeys are one of the most neglected primate subfamilies. Although various classifications and phylogenetic relationships among colobine genera, mainly based on morphological characteristics, were proposed, their evolutionary history is still controversially discussed. In recent years, an increasing number of molecular studies have been carried out, but due to contradicting gene trees, elucidating the real relationships among colobine genera became even more complicated. Insufficient data, homoplasy, differential lineage sorting or hybridization are possible explanations for gene tree discordance. However, hybridization as possible reason is traditionally neglected, since its effect in speciation processes is regarded as minimal, at least in animals. To further illuminate the evolutionary and biogeographic history of colobine genera and to determine the reasons for gene tree incongruences, we combined presence/absence analyses of Alu insertions with autosomal, $\mathrm{Y}$ chromosomal and mitochondrial sequence data (in total $\sim 25,000 \mathrm{bp}$ ).

Results and conclusions: According to our data, a common origin of colobines, as well as of solely Asian representatives and of odd-nosed monkeys is strongly supported. However, different data sets varied in positioning Piliocolobus among colobines, and concerning the relationships among the odd-nosed monkey clade and the three langur genera Presbytis, Semnopithecus and Trachypithecus. Hence, the monophyly of African colobines and the langur group is questioned. Although homoplasy, inaccurate data and incomplete lineage sorting might have influenced gene tree topologies to some degree, they provide no sufficient explanation for the observed discordance. Hence, hybridization as main reason is favoured, which might have occurred between the two African genera Colobus and Piliocolobus and the two Asian langur genera Semnopithecus and Trachypithecus. Based on phylogenetic relationships and estimated divergence ages, colobines most likely originated in Africa. In the late Miocene, they invaded Asia and diversified within a relative short time period into respective genera. Genetic exchange between Trachypithecus and Semnopithecus occurred until the late Pliocene.
\end{abstract}

\title{
5.1 Introduction
}

Gene trees do not necessarily reflect species trees and even discrepancies among various gene trees are common. Homoplasy, insufficient data, differential lineage sorting, or introgressive hybridization are possible explanations for these incongruences (Avise, 2000; Barton, 2001; Hewitt, 2001; Funk and Omland, 2003; Seehausen, 2004). Accumulating evidence suggest that natural hybridization as one reason seems to be more common than previously thought. However, its implications for evolutionary processes are still unclear, at least in animals (Grant and Grant, 2002; Seehausen, 2004; Grant et al., 2005; Jorgensen and Mauricio, 2005; Arnold and Meyer, 2006; Nolte et al., 2006). Is gene flow through hybrid populations really as ineffective in speciation as it was assumed to be (Darwin, 1859; Mayr, 1942, 1963)? Several studies proposed reticulate evolution or hybridization events in various taxa, acting as driving forces for novel traits and diversification (McCracken and Sorenson, 2005; Berthier et al., 2006; Patterson et al., 2006; Pidancier et al., 2006; Koblmüller et al., 2007; Bicca-Marques et al., 2008; McDonald et al., 2008). Also for primates, several examples of natural hybridization between species (e.g. Eulemur sp., Wyner et al., 2002; Lepilemur sp., Rumpler et al., in press; Alouatta sp., Cortes-Ortiz et al., 2007; Macaca sp., Evans et al., 2003; Papio sp., Alberts and Altmann, 2001; Gorilla sp., Thalmann et al., 2007) and even the formation of new species as result of hybridization (e.g. Macaca arctoides, Tosi et al., 2000; Macaca munzala, Chakraborty et al., 2007) are 
known (for review see Arnold and Meyer, 2006). However, evidence for introgressive hybridization between genera of free-ranging primates was only found between Semnopithecus and Trachypithecus (Osterholz et al., 2008), Theropithecus and Papio (Jolly et al., 1997) and the early hominine and chimpanzee lineages shortly after they initially diverged from each other (Patterson et al., 2006). These intra- and intergeneric examples suggest that natural hybridization may indeed play a role in primate speciation and diversification, but its actual impact has to be further investigated.

Colobines (subfamily Colobinae) are one of the genetically least studied primate taxa. Only a few molecular studies have been published and sequence data are limited. The classification of colobine genera and their phylogenetic relationships are still disputed and numerous controversial phylogenetic hypotheses have been proposed. Recent studies detected substantial incongruences among gene trees of some Asian colobine genera, which might be caused by ancestral hybridizations or other effects as mentioned above (Osterholz et al., 2008; Ting et al., 2008). However, a genetic analysis of colobine monkeys including sequence data from maternal-, paternal- and biparentalinherited loci or the presence/absence pattern of SINE insertions is still lacking.

Colobines form a diverse group of Old World monkeys. They share some peculiar autapomorphic features, which make them unique among primates and provide evidence for a monophyletic origin. Most prominent is the complex, at least three-chambered and ruminant-like stomach, which enables colobine monkeys to digest celluloses (Chivers and Hladik, 1980). Further typical characteristics include a high molar relief, elongated legs and tails and a reduced or even absent pollex (Chivers and Hladik, 1980; Strasser and Delson, 1987). Colobines are distributed in Africa and Asia and they most likely diverged from their closest relatives, the cheek-pouched monkeys (subfamily Cercopithecinae) in the mid-Miocene (Delson, 1994; Sterner et al., 2006).

Colobines are traditionally arranged into an African and an Asian clade, mainly based on morphological traits and geographic distribution (Napier, 1970; Szalay and Delson, 1979; Oates et al., 1994; Groves, 2001). African colobines have been further split into the three genera Colobus, Procolobus and Piliocolobus (Groves, 2001), while the latter two are sometimes combined in the genus Procolobus (Pocock, 1936; Kuhn, 1967; Oates et al., 1994; Grubb et al., 2003). The three African genera are believed to form a monophyletic group (Groves, 2001; Napier, 1970; Oates et al., 1994; Szalay and Delson, 1979), but paraphyly was also proposed (Jablonski, 1998). Molecular studies including all three African genera are limited. However, those in which besides Colobus also Procolobus and/or Pilicolobus were included, suggest a common origin of African colobines (Messier and Stewart, 1997; Sterner et al., 2006; Ting, 2008; Ting et al., 2008).

Asian colobines are more diverse than their African relatives and various alternative classifications have been proposed (Pocock, 1935, 1939; Napier and Napier, 1967; Brandon-Jones, 1984; Oates et al., 1994; Brandon-Jones et al., 2004). According to Groves (2001), the most suitable classification of Asian colobines is the division into the odd-nosed monkey group, comprising the 
genera Pygathrix, Rhinopithecus, Nasalis and Simias, and the langur group, with Semnopithecus, Trachypithecus and Presbytis. A common origin of Asian colobines is well supported by chromosomal (Bigoni et al., 2003; Bigoni et al., 2004) and molecular studies (Collura et al., 1996; Messier and Stewart, 1997; Stewart and Disotell, 1998; Zhang and Ryder, 1998; Page et al., 1999; Xing et al., 2005; Sterner et al., 2006; Whittaker et al., 2006; Karanth et al., 2008; Osterholz et al., 2008; Ting et al., 2008). Likewise the monophyly of the odd-nosed monkey group is widely accepted and confirmed by morphological (Bennett and Davies, 1994; Groves, 2001) and genetic data (Sterner et al., 2006; Osterholz et al., 2008; Ting et al., 2008). In contrast to recent genetic data, which show that Simias and Nasalis are closely related and that both are nested within the odd-nosed monkey clade (Whittaker et al., 2006), earlier studies regarded Simias alone or clustered with Nasalis as basal among colobines (Groves, 1989; Jablonski, 1998).

The putative sister clade of the odd-nosed monkeys are the langurs. Its common origin was mainly inferred from morphological traits, reflected also by early classifications in which all three genera were combined in a single genus (Presbytis or Semnopithecus) (Napier and Napier, 1967; Groves, 1970; Delson, 1975). However, molecular evidence for the monophyly of langurs is still lacking. Nuclear and mitochondrial data revealed contrasting relationships among langur genera and the oddnosed monkey group (Zhang and Ryder, 1998; Sterner et al., 2006; Osterholz et al., 2008; Ting et al., 2008). Morphological similarities between Semnopithecus and Trachypithecus lead to the exclusion of Presbytis (Brandon-Jones, 1984; Strasser and Delson, 1987; Groves, 2001), a pattern which is also supported by nuclear data (Osterholz et al., 2008; Ting et al., 2008). However, mitochondrial sequence data do either not resolve these relationships (Osterholz et al., 2008) or even suggest a clade consisting of Presbytis and Trachypithecus (Sterner et al., 2006).

SINE (Short INterspersed Element) insertions, in particular the primate-specific Alu elements, have proven to be useful at various levels of phylogenetic analyses and are widely applied as a powerful molecular-cladistic tool (Schmitz et al., 2001; Roos et al., 2004; Ray et al., 2005; Schmitz et al., 2005; Xing et al., 2005; Herke et al., 2007; Xing et al., 2007; Osterholz et al., 2008). The probability of an Alu element to insert independently into the same locus in different genomes is insignificant and a true orthology can be verified by their flanking direct repeats. Furthermore, the insertion is irreversible and the precise removal is extremely unlikely (Shedlock and Okada, 2000; Schmitz et al., 2005; van de Lagemaat et al., 2005). Consequently, the lack of an Alu element at a certain locus can be considered to be the ancestral state and therefore a common ancestor can be determined by a shared insertion (Okada, 1991; Shedlock and Okada, 2000; Schmitz et al., 2005). Based on these characteristics, SINE insertions can be regarded as noise-free Hennigian synapomorphies (Shedlock and Okada, 2000).

To further elucidate the supposed complex evolutionary history of colobine monkeys, we examined the presence/absence pattern of SINE insertions and compared the inferred phylogeny with 
that derived from mitochondrial and nuclear sequence data. To detect possible hybridization events among genera, we analysed maternal-, paternal- and biparental-inherited loci. Accordingly, five autosomal loci, which map to different human chromosomes, and six Y chromosomal loci were PCRamplified and sequenced. As maternal-inherited marker, we reanalysed available mitochondrial genome data.

\subsection{Results}

\subsubsection{Alu insertions}

In total, 189 formerly undetected Alu insertion loci were tested for presence or absence of respective insertions. Among them, only 13 loci proved to be phylogenetically informative. All other Alu elements were present only in a single genus or the amplification in essential genera was not possible. To increase the number of informative loci, another 153 insertions known from earlier studies were included (Salem et al., 2003; Xing et al., 2005; Osterholz et al., 2008), of which some were further examined in previously untested genera (Supplementary table 5.2). The orthology of insertions was confirmed by sequencing. In all cases, direct repeats flanking the insertion as well as the original target site prior transposition were detected. Moreover, no insertions showing conflicting relationships were found.

Based on these insertions, an unequivocal phylogeny of colobine genera and outgroup taxa could be established (Fig. 5.1A). The sister grouping of Homo and Pan (A-I) is supported by 16 insertions (Salem et al., 2003). Among Cercopithecinae 15 insertions support a common origin of Macaca and Papio (A-II) and the clade consisting of the latter two genera and Chlorocebus (A-III) is supported by 33 insertions (Xing et al., 2005). The monophyly of all Cercopithecidae representatives (A-IV) is supported by 35 insertions (Xing et al., 2005).

20 Alu insertions (A-V) unite all colobines and strongly support their monophyly. Among colobines, three unlinked Alu insertions (A-VI) were found in Piliocolobus and all Asian colobines, but not in Colobus and hence, a paraphyly of African colobines is indicated. A common origin of solely Asian genera (A-VII) is supported by 26 Alu insertions. Among Asian colobines, ten Alu elements unite Semnopithecus and Trachypithecus (A-VIII) to the exclusion of all other colobines, while two insertions link Presbytis with Nasalis/Pygathrix (A-IX). The monophyly (A-X) of the latter two genera is supported by six insertions. 


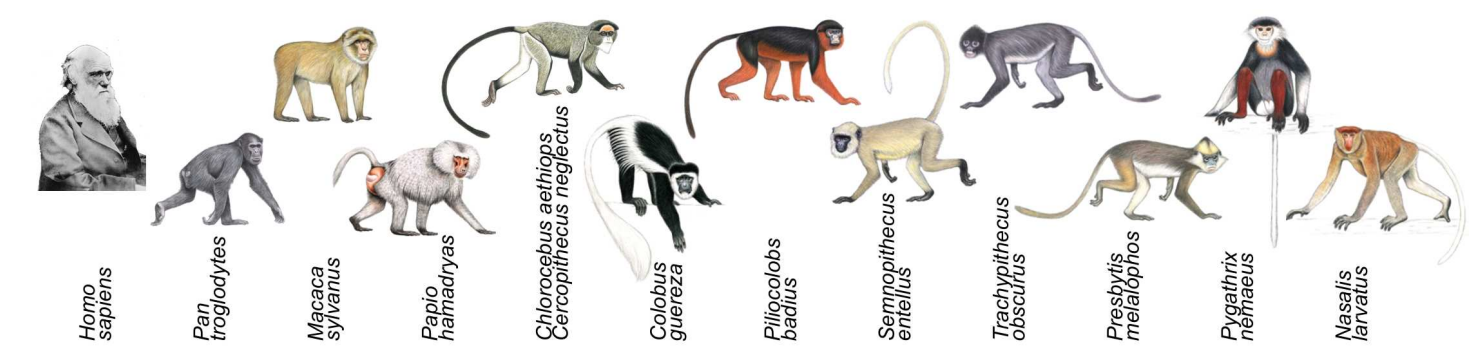

A
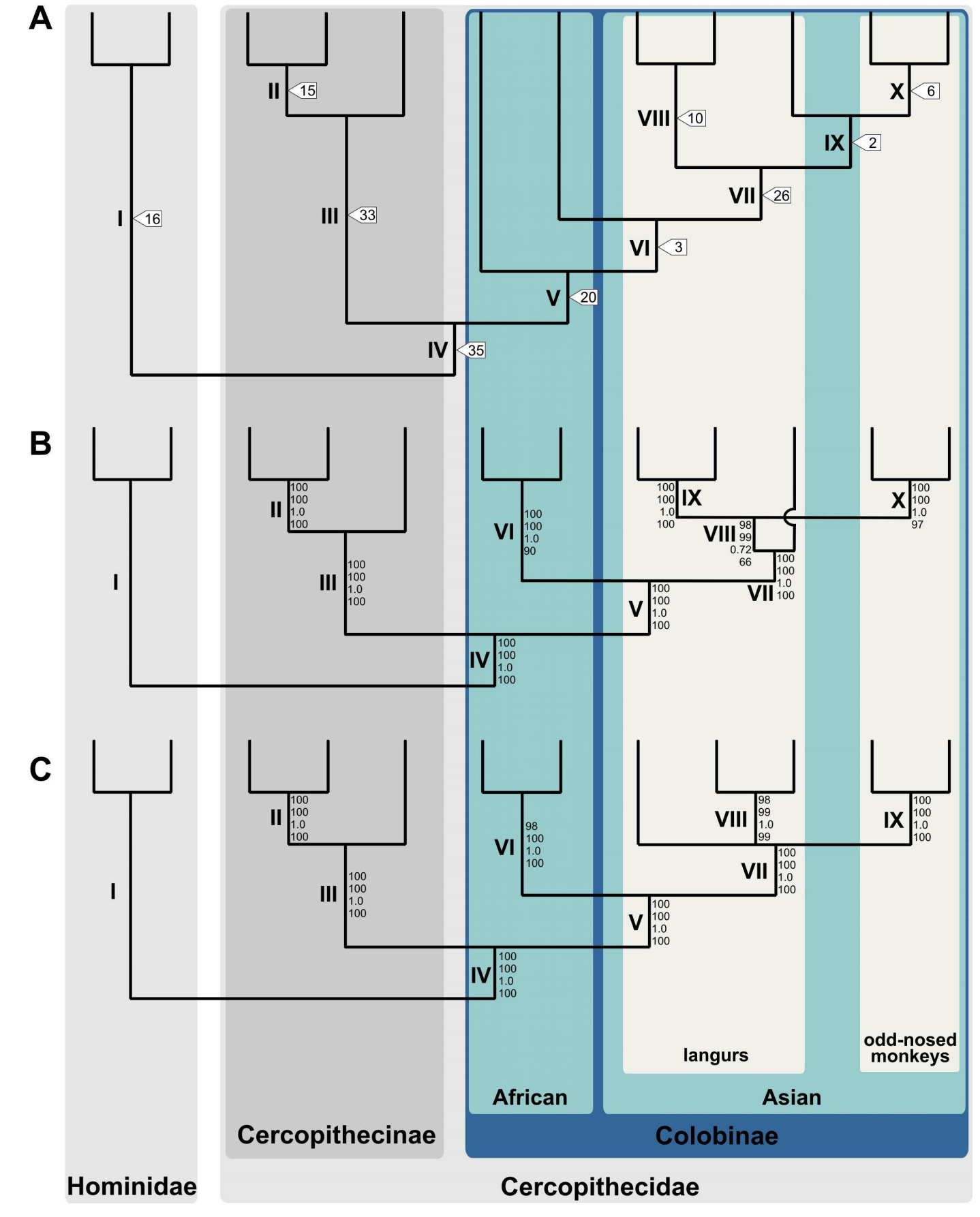

Figure 5.1: Phylogenetic relationships among colobine genera and outgroup taxa as inferred from (A) Alu insertion loci, (B) nuclear sequence data, and (C) mitochondrial genome data. Numbers in flags and beside branches indicate the number of Alu insertions and bootstrap support or posterior probability values (top to bottom: MP, NJ, Bayesian, ML), respectively. 


\subsubsection{Nuclear phylogeny}

Our complete nuclear data set, including five autosomal and six $\mathrm{Y}$ chromosomal loci, comprises $8,917 \mathrm{bp}$, of which 1,291 sites are variable and 722 parsimony-informative. Phylogenetic analyses yielded identical and for most cases significantly supported branching patterns among studied taxa irrespectively of the applied algorithm (maximum-parsimony: MP, neighbor-joining: NJ, maximumlikelihood: ML, Bayesian). Tree topologies for individual loci or concatenated autosomal and $Y$ chromosomal data sets were in general congruent with the tree derived from the combined data, but in some cases no resolution of relationships were obtained and support values were in general lower (data not shown).

Based on nuclear data (Fig. 5.1B), significant support was obtained for a common origin of Cercopithecidae (B-IV) to the exclusion of hominids (B-I) and its division into Cercopithecinae (B-III) and Colobinae (B-V). Among cercopithecines, Macaca and Papio (B-II) form a strongly supported sister clade to Cercopithecus. Among colobines, African (B-VI) and Asian (B-VII) colobine genera form reciprocal monophyletic groups, a division, which is significantly supported by MP, NJ and Bayesian algorithms, but ML bootstrap values for a sister grouping of the two African genera do not exceed 90\%. The Asian genera further diverged into a lineage leading to Presbytis and another to all the remaining genera (B-VIII), which later split into two clades, with one consisting of Trachypithecus and Semnopithecus (B-IX) and the second of Nasalis and Pygathrix (B-X). Both clades are strongly supported.

Although most relationships are significantly supported, some of them are not in agreement with the tree topology as obtained from presence/absence data of Alu elements. Accordingly, we tested the reliability of the depicted relationships by evaluating alternative tree topologies with the KishinoHasegawa and Shimodaira-Hasegawa tests. Based on these data, a paraphyly of African colobines with Piliocolobus being closer related to Asian colobines than to Colobus as proposed by three Alu insertions is not rejected $(\mathrm{P}>0.05)$. Traditionally, Presbytis is recognized as member of the langur group, which also includes Semnopithecus and Trachypithecus, but nuclear data indicate a basal position of Presbytis among Asian colobines and two Alu insertions support a close relationship to the oddnosed monkeys. Alternative positions of Presbytis among Asian colobines, in which all these proposed relationships were tested, are not rejected. However, a close affiliation of Presbytis to either Semnopithecus or Trachypithecus is rejected on a significance level of $\mathrm{P}<0.005$.

Based on these findings, it is highly likely that nuclear sequence data do not reflect the real relationships among colobine monkeys, which might be caused by the relative low number of polymorphic sites. Hence, divergence ages from nuclear sequence data were estimated on the basis of an a priori fixed tree topology as proposed by Alu insertions. Accordingly, the major split among cercopithecids leading to the two subfamilies Colobinae and Cercopithecinae occurred $\sim 17.4 \pm 1.4$ million years ago (mya), while among the latter Cercopithecus diverged from the Macaca/Papio clade 
$\sim 9.6 \pm 0.9$ mya (Table 5.1). Among colobines, Colobus and Piliocolobus split off from the Asian genera $\sim 10.7 \pm 1$ mya and $\sim 10.4 \pm 1$ mya, respectively. The Asian lineage further diverged $\sim 7.6 \pm 0.8$ mya into a group consisting of Trachypithecus/Semnopithecus and a clade with Presbytis, Nasalis and Pygathrix. Among the latter, Presbytis split off $\sim 7.6 \pm 0.8$ mya, before finally also Nasalis and Pygathrix diverged $\sim 5.7 \pm 0.8$ mya. The most recent split among Asian genera occurred between Trachypithecus and Semnopithecus $(\sim 2.3 \pm 0.5$ mya).

Table 5.1: Estimated divergence times

\begin{tabular}{lcc}
\hline Divergence & $\begin{array}{c}\text { nucDNA } \\
\text { HKY }+\mathrm{G}, \mathrm{C3}\end{array}$ & $\begin{array}{c}\text { mtDNA } \\
\text { GTR+l+G, C3 }\end{array}$ \\
\hline Homo-Pan & $6.1 \pm 0.2$ & $6.0 \pm 0$ \\
Cercopithecoidea - Hominoidea & $34.3 \pm 0.5$ & $28.2 \pm 1.7$ \\
Cercopithecinae - Colobinae & $17.4 \pm 1.4$ & $17.1 \pm 0.9$ \\
Cercopithecini - Papionini & $9.6 \pm 0.9$ & $10.1 \pm 0.5$ \\
Papio- Macaca & $6.3 \pm 0.3$ & $7.9 \pm 0.1$ \\
Colobus - (Piliocolobus, Asian colobines) & $10.7 \pm 1$ & - \\
Piliocolobus - Asian colobines & $10.4 \pm 1$ & - \\
African colobines - Asian colobines & - & $11.1 \pm 0.6$ \\
Colobus - Piliocolobus & - & $8.3 \pm 0.6$ \\
Asian split & $7.6 \pm 0.8$ & $8.8 \pm 0.4$ \\
Trachypithecus - Semnopithecus & $2.3 \pm 0.5$ & - \\
Presbytis - odd-nosed monkeys & $7.6 \pm 0.8$ & - \\
Presbytis - Trachypithecus & - & $7.7 \pm 0.5$ \\
Nasalis - Pygathrix & $5.7 \pm 0.8$ & $6.4 \pm 0.2$ \\
\hline
\end{tabular}

\subsubsection{Mitochondrial phylogeny}

Among the 15,905 bp in the mitochondrial alignment, 6,995 sites are variable and 5,131 of them parsimony-informative. Phylogenetic relationships among genera as suggested by various algorithms are mainly congruent and significantly supported (Fig. 5.1C). In detail, the data strongly support a Cercopithecidae clade (C-IV) to the exclusion of Homo + Pan (C-I), the monophyly of the two cercopithecid subfamilies Colobinae (C-V) and Cercopithecinae (C-III), and among the latter, a sister grouping of Chlorocebus to a Papio/Macaca clade (C-II). Colobines initially diverged into an African (CVI) and an Asian (C-VII) clade. The Asian colobine clade further splits into a lineage leading to Pygathrix/Nasalis (C-IX), a lineage comprising Trachypithecus and Presbytis (C-VIII), and finally a lineage with Semnopithecus. The relationships among these three lineages are not well resolved. While maximum-parsimony, neighbor-joining and Bayesian approaches weakly support a sister grouping of Semnopithecus to the Presbytis/Trachypithecus clade, the maximum-likelihood inference indicates a clade 
consisting of Semnopithecus and the Nasalis/Pygathrix clade (data not shown). Finally, the monophyly of both the Nasalis/Pygathrix and Presbytis/Trachypithecus clades are again strongly supported.

Due to incongruences between nuclear and mitochondrial trees, alternative tree topologies were also tested for the mitochondrial data set. A paraphyly of African colobines with Piliocolobus being closer related to Asian colobines than to Colobus, as indicated by Alu insertions, is significantly rejected $(\mathrm{P}<0.001)$. Nuclear and mitochondrial tree topologies differ also concerning the relationships among the three langur genera Semnopithecus, Trachypithecus and Presbytis, and the odd-nosed monkey clade. The tests revealed, that relationships in which Trachypithecus and Presbytis do not form a monophyletic group are significantly rejected $(\mathrm{P}<0.005)$ and also the close relationship of Trachypithecus and Semnopithecus as suggested by nuclear data is rejected $(\mathrm{P}<0.005)$. In contrast, different positions of Semnopithecus among Asian colobines as the grouping with either the Trachypithecus/Presbytis or the Nasalis/Pygathrix clade, as well as a basal position or an unresolved trichotomy among these three lineages are not rejected $(\mathrm{P}>0.05)$. Hence, an unresolved trichotomy might best reflect the relationships among these three lineages in the mitochondrial tree.

As for the nuclear data set, divergence ages for the mitochondrial genome data were estimated on the basis of an a priori fixed tree topology. Relationships were in general in agreement with those depicted in Fig. 5.1C, with exception of the unresolved trichotomy among the three major Asian lineages. Based on the estimates (Table 5.1), the initial split among cercopithecids leading to the two subfamilies Colobinae and Cercopithecinae occurred $17.1 \pm 0.9$ mya. Among Cercopithecinae, Chlorocebus diverged from the Macaca/Papio clade $10.1 \pm 0.5$ mya, while the latter split into its respective genera $\sim 7.9 \pm 0.1$ mya. Colobines initially split into an African and an Asian lineage $\sim 11.1 \pm$ 0.6 mya. The two African genera finally diverged from each other $\sim 8.3 \pm 0.6$ mya. The major Asian split leading to the three lineages Semnopithecus, Trachypithecus/Presbytis and Nasalis/Pygathrix occurred $\sim 8.8 \pm 0.4$ mya. The latter two clades further diverged into respective genera $\sim 7.7 \pm 0.5$ and $\sim 6.4 \pm 0.2$ mya.

\subsection{Discussion}

By combining presence/absence analyses of Alu integration with extensive autosomal, Y chromosomal and mitochondrial sequence data (in total $\sim 25,000 \mathrm{bp}$ ), the present study provides new and comprehensive insights into the evolutionary history of colobine monkeys. Most relationships are resolved and strongly supported by various algorithms and Alu insertions, and the branching patterns for which statistical support is relative low, are evidently confirmed by Alu insertions. Moreover, divergence ages and relationships as revealed from the different data sets are mainly congruent and in agreement with estimates from other molecular studies (Collura et al., 1996; Stewart 
and Disotell, 1998; Zhang and Ryder, 1998; Raaum et al., 2005; Xing et al., 2005; Sterner et al., 2006; Karanth et al., 2008; Osterholz et al., 2008; Ting, 2008; Ting et al., 2008).

Identical branching patterns among different gene trees were obtained for the division of Cercopithecidae into two subfamilies (Colobinae, Cercopithecinae), a sister grouping of the Macaca/Papio clade to Cercopithecini, represented by either Cercopithecus or Cholorocebus, and a common origin for both an Asian colobine, and an odd-nosed monkey clade within the Asian colobines. Also divergence times for respective splits are comparative among gene trees and to previous data (Goodman et al., 1998; Stewart and Disotell, 1998; Page et al., 1999; Raaum et al., 2005; Steiper and Young, 2006; Sterner et al., 2006; Ting, 2008). Accordingly, the major split among cercopithecids occurred $\sim 17.5 \pm 1.4$ mya. The Macaca/Papio clade diverged from Cercopithecini $\sim 9.7 \pm 1$ mya, while the last common ancestor of Asian colobines and odd-nosed monkeys lived $\sim 8.1 \pm 1.2$ mya and $\sim 5.8 \pm 0.8$ mya, respectively.

However, our study revealed also massive discrepancies among gene trees. First, mitochondrial data significantly support a sister grouping of the two African colobine genera Colobus and Piliocolobus, whereas Alu insertions provide evidence for a closer affiliation of Piliocolobus to Asian colobines than to Colobus. Although nuclear sequence data support the mitochondrial pattern, an alternative relationship with Piliocolobus forming a sister genus to Asian colobines to the exclusion of Colobus is not rejected. Accordingly, mitochondrial DNA supports the monophyly of African colobines, while nuclear data indicate their paraphyly. The observed discrepancy becomes also obvious by considering the estimated divergence ages. Based on mitochondrial data, African and Asian colobines diverged $\sim 11.1 \pm 0.6$ mya and the split between Colobus and Piliocolobus occurred $\sim 8.3$ \pm 0.6 mya. In contrast, the nuclear-based estimates indicate a separation of Colobus from Piliocolobus and Asian colobines $\sim 10.7 \pm 1$ mya, and a division of the latter two shortly after, $\sim 10.4 \pm 1$ mya.

The second example for discrepancies among gene trees concerns the relationships among the three langur genera Presbytis, Semnopithecus and Trachypithecus, and the odd-nosed monkey group. Nuclear sequence data and Alu insertions support close relationships between Semnopithecus and Trachypithecus, and between Presbytis and the odd-nosed monkeys. The latter clade is mainly supported by two Alu insertions and nuclear sequence data do not contradict this hypothesis. In contrast to nuclear data, mitochondrial DNA strongly links Trachypithecus with Presbytis. The position of Semnopithecus relative to the clades Presbytis/Trachypithecus and odd-nosed monkeys is not resolved. Hence, the mitochondrial relationships among the three Asian lineages Semnopithecus, Presbytis/Trachypithecus and odd-nosed monkeys might be best displayed as trichotomic split. Based on nuclear sequence data, the split between Semnopithecus/Trachypithecus and Presbytis/odd-nosed monkeys occurred $\sim 7.6 \pm 0.8$ mya. Presbytis diverged from the odd-nosed monkeys shortly afterwards, while Trachypithecus and Semnopithecus had a common ancestor until $2.3 \pm 0.5$ mya. According to mitochondrial DNA, the initial split among Asian colobines into the three lineages Semnopithecus, 
Presbytis/Trachypithecus and odd-nosed monkeys occurred $\sim 8.8 \pm 0.4$ mya, while Presbytis and Trachypithecus diverged from each other $\sim 7.7 \pm 0.5$ mya.

Inadequate data, homoplasy, incomplete lineage sorting or hybridization are possible explanations for the observed incongruence (Avise, 2000; Funk and Omland, 2003). For the mitochondrial data set, at least for the Colobus/Piliocolobus and Presbytis/Trachypithecus clades, incorrect branching patterns due to inadequate data or homoplasy are unlikely, since a sufficient phylogenetic resolution with long branches was obtained. However, for the unresolved position of Semnopithecus among Asian colobines, inadequate data are the most likely explanation. Differential sorting of ancestral mitochondrial lineages can also be excluded. If the Colobus/Piliocolobus and Presbytis/Trachypithecus clades would be indeed the result of incomplete sorting of mitochondrial lineages, the mitochondrial divergence between respective genera should predate the nuclear splitting times, which is not the case. For the nuclear data set, homoplasy, incomplete lineage sorting and insufficient data can be excluded as well, at least for the branching of Trachypithecus and Semnopithecus, because ten independent Alu insertions and sequence data from six Y chromosomal and five unlinked autosomal loci clearly confirm their close relationship. However, the ambiguous relationships of Piliocolobus and Presbytis among colobines in the nuclear sequence data set might be affected by homoplasy, but insufficient data might be an alternative explanation therefore. Hence, the positioning of Presbytis and Piliocolobus among colobines does not rely on nuclear sequence data, but mainly on Alu insertions. Homoplasy and incomplete lineage sorting for Alu insertions is unlikely, because the probability that several unlinked Alu elements integrate independently at orthologous sites in different genomes is implausible and neglectable (Shedlock and Okada, 2000; Schmitz et al., 2005).

Although inadequate data, homoplasy and differential lineage sorting may have affected the tree topologies to some degree, these factors provide no sufficient explanation for the herein detected gene tree discordances and hence, ancestral hybridization events as possible reason are favoured therefore. A hybridization hypothesis gains further support by some natural characteristics as behavior or morphology, or distribution.

Hybridization between the two African genera Colobus and Piliocolobus is in principal possible, because they occur sympatrically over wide ranges of their distribution (Oates et al., 1994). Furthermore, in contrast to Colobus and most other primates, females in Piliocolobus tend to leave their natal groups (Newton and Dunbar, 1994; Oates et al., 1994) and finally, Colobus males are in average larger than males in Piliocolobus (Oates et al., 1994). Accordingly, we propose a scenario in which proto-Colobus was separated from the main colobine stem $\sim 10.7 \pm 1$ mya, shortly afterwards followed by proto-Piliocolobus. After these initial splits, a limited number of Piliocolobus females hybridized with Colobus males leading to female (mitochondrial) introgression of Piliocolobus into Colobus. By backcrossing of hybrid females with Colobus males, the Piliocolobus mitochondrial lineage became 
fixed in Colobus, while the original nuclear lineages of Colobus increased in every generation. Based on our calculations, this hybridization event occurred $\sim 8.3 \pm 0.6$ mya, although the possibility that hybridization took place over a longer time period, beginning directly or sometime after the initial split $\sim 10.7 \pm 1$ mya until the divergence of the mitochondrial lineages, can not be excluded.

Ancestral hybridization seems to be also the most plausible explanation for the gene tree incongruences among Asian colobines. The close relationship between Semnopithecus and Trachypithecus is also supported by morphological similarities, and hybridization events due to partly overlapping distribution zones are in general possible (Oates et al., 1994; Groves, 2001; Brandon-Jones et al., 2004). Accordingly, in an initial split, Asian colobines diverged $~ 8.1 \pm 1.2$ mya into Semnopithecus and a progenitor of Presbytis, Trachypithecus and the odd-nosed monkeys. Among the latter, the progenitor of odd-nosed monkeys split off shortly after the initial division, while Presbytis and Trachypithecus were separated from each other $\sim 7.7 \pm 0.5$ mya. Semnopithecus males, which are much larger than males of Trachypithecus (Oates et al., 1994), hybridized with Trachypithecus females, leading to the fixation of mitochondrial DNA in hybrids. By backcrossing with pure Semnopithecus males over a long period (until $\sim 2.3 \pm 0.5$ mya), the progenitor of Trachypithecus accumulated solely nuclear material of Semnopithecus (nuclear swamping), while the mitochondrial genome remained Trachypithecus-like.

Based on phylogenetic relationships and estimated divergence ages, colobines most likely originated in Africa, which is in agreement with earlier suggestions (Stewart and Disotell, 1998). Support for this hypothesis is provided by the fact that with exception of macaques, which mainly occur in Asia, all other recent members of the sister lineage to colobines, the cercopithecines, are solely distributed in Africa. Another strong hint for an African origin is provided by the paraphyly of African colobines, because a putative Asian origin would require two migration steps into Africa. According to our data, colobines and cercopithecines were separated from each other $\sim 17.5 \pm 1.4$ mya. Among colobines, Colobus split off first from the main stem ( 10.7 \pm 1 mya). Shortly afterwards, in a time frame of only $\sim 200,000$ years, also Piliocolobus diverged from the progenitor of Asian colobines. Although initially separated, hybridization events between Piliocolobus and Colobus occurred until $\sim 8.3$ \pm 0.6 mya. The progenitor of Asian colobines most likely invaded Eurasia via an emerging landbridge connection between Africa and the Arabian Peninsula in the late Miocene (Whybrow, 1984, 1992; Stewart and Disotell, 1998), sometimes after the separation from Piliocolobus $10.4 \pm 1$ mya and before its diversification $\sim 8.1 \pm 1.2$ mya. Whether a route north or south of the Himalaya was selected is putative, but at least north of the Himalaya, on the Tibetan plateau, colobine fossils from the late Miocene were found, but not south of the Himalaya (Delson, 1994). The diversification hotspot of Asian colobines is unknown, but the Hengduan Mountains in the border region of today's Burma, India and China might have been a possible place (Peng et al., 1993; Jablonski, 1998). This is supported by the fact that all of the larger Southeast-Asian rivers (Mekong, Salween, Yangtze) emerge there, 
which are well-known to act as barriers for arboreal primates (Meijaard and Groves, 2006). As first lineage, Semnopithecus diverged from the main stem and invaded the Indian subcontinent. Shortly afterwards, also the progenitor of Presbytis and Trachypithecus separated from the odd-nosed monkey ancestor and migrated into southern mainland Asia. Finally, Presbytis entered Sundaland and diverged from the southern mainland form Trachypithecus $\sim 7.7 \pm 0.5$ mya. The odd-nosed monkeys also split into different lineages, which further expanded their range into Indochina and Sundaland $\sim 5.8 \pm 0.8$ mya. Trachypithecus and Semnopithecus came into secondary contact and exchanged genetic material until $\sim 2.3 \pm 0.5$ mya, most likely in the region of today's Bangladesh, Burma and the Northeast of India.

\subsection{Conclusions}

Our study gives new and detailed insights into the evolutionary and biogeographic history of colobine monkeys, and provides evidence for hybridization events among ancestral genus lineages. The analysis of presence/absence patterns of Alu insertions proved to be the most reliable tool to reconstruct phylogenetic relationships, although also pure sequence data contributed to some degree. However, as this study has shown, Alu insertion data alone are not sufficient to confirm putative hybridization events, because data from maternal-, paternal- and biparental inherited loci are required to fully elucidate such events. Hybridization among taxa is traditionally recognized as factor, which leads to limited diversification, reproductive isolation and lowered fitness (Darwin, 1859, Mayr, 1963). However, our and other data clearly support that hybridization plays a role in the diversification of primates, although its extend has to be further investigated.

\subsection{Experimental Procedures}

\subsubsection{Samples}

Total genomic DNA was extracted from blood or tissue samples using the DNeasy Blood \& Tissue Kit from Qiagen. Samples were obtained from Colobus guereza (Guereza, Cologne Zoo, Germany), Piliocolobus badius (Western red colobus, Taï National Park, Ivory Coast; provided by Linda Vigilant, Max-Planck-Institut Leipzig, Germany), Semnopithecus entellus (Hanuman langur, Dresden Zoo, Germany), Trachypithecus obscurus (Dusky leaf monkey, Wuppertal Zoo, Germany), Presbytis melalophos (Sumatran surili, Howletts Wild Animal Park, UK), Pygathrix nemaeus (Red-shanked douc langur, Cologne Zoo, Germany), Nasalis larvatus (Proboscis monkey, Wilhelma Stuttgart, Germany; provided by Werner Schempp, University of Freiburg, Germany), Macaca sylvanus (Barbary macaque, 
Nuremberg Zoo, Germany), Papio hamadryas (Hamadryas baboon, Munich Zoo, Germany), Cercopithecus neglectus (De Brazza's Monkey, Leipzig Zoo, Germany), Chlorocebus aethiops (Grivet, provided by Roland Plesker, Paul-Ehrlich-Institut Langen, Germany) and Pan troglodytes (Chimpanzee, Munich zoo, Germany). The only colobines not included in this study are Simias, Rhinopithecus and Procolobus, since all of them are closely related to other genera (Groves, 1970; Delson, 1975; Whittaker et al., 2006). Outgroup taxa include representatives from all relevant catarrhine groups (Homo, Pan, Macaca, Papio, Cercopithecus or Chlorocebus). For a more comprehensive analysis, further sequences deposited at GenBank were added to the data sets.

\subsubsection{Alu insertions}

Due to their high copy number and relative small size $(\sim 300 \mathrm{bp})$, the primate specific Alu elements were selected as molecular-cladistic markers. The presence or absence of Alu elements in different colobines at specific loci was tested via PCR using primers occupying the flanking region of the insertion site. The orthology of insertions was confirmed by sequencing. Details on analysed loci, primers and presence/absence pattern of Alus in studied species are listed in supplementary table 5.2.

To detect new Alu insertion loci, a subtractive hybridization approach was performed as described in Mamedov et al. (2005) and Diatchenko et al. (1996), but with some modifications (Osterholz et al., 2008). To avoid biased hybridization results, various species combinations were used as tracer and driver (hybridization (hyb.) 1: tracer Nasalis/Pygathrix, driver Presbytis; hyb. 2: tracer Nasalis/Pygathrix, driver Semnopithecus; hyb. 3: tracer Trachypithecus/Presbytis, driver Pygathrix; hyb. 4: tracer Presbytis, driver Semnopithecus). A detailed description of respective laboratory methods is outlined in Osterholz et al. (2008). To increase the number of informative loci, another 153 insertions (Salem et al., 2003; Xing et al., 2005; Osterholz et al., 2008) were included, of which colobine markers were further examined in previously untested genera (Supplementary table 5.2).

\subsubsection{PCR and sequencing of nuclear loci}

Exonic, but mainly intronic sequences were generated for six single-copy genes mapping to the non-recombining portion of the $\mathrm{Y}$ chromosome, and for five autosomal loci, which map to different human chromosomes. With exception of the SRY gene (sex-reversal, Y chromosome), all other Y chromosomal loci (DBY5: Dead Box, intron 5; SMCY7: SMC mouse homologue, intron 7; SMC11: SMC mouse homologue, intron 11; UTY18: ubiquitous TPR motif, intron 18; ZFYLI: Zinc finger, last intron) have homologues on the $\mathrm{X}$ chromosome ( $\mathrm{X}$ degenerate). $\mathrm{PCR}$ conditions and primers for amplifying $\mathrm{Y}$ chromosomal loci were applied as listed in supplementary table 5.1. Intron 11 of the von Willebrand Factor (vWF11), located on the short arm of human chromosome 12, was amplified following methods as described in Chaves et al. (1999). For intron 3 of the serum albumin gene (ALB3, human chromosome 4), intron 3 of the interstitial retinol-binding protein, (IRBP3, human chromosome 10), 
intron 1 of the transition protein 2 (TP2, human chromosome 16) and intron 1 of the transthyretin gene (TTR1, human chromosome 18), new primers were designed on the basis of available primate sequences in GenBank. Oligonucleotide primers, PCR conditions and the size of PCR products are listed in supplementary table 5.1. The results of the PCR amplifications were checked on agarose gels. PCR products were cleaned with the Qiagen PCR Purification Kit and subsequently sequenced on an ABI 3730xl sequencer using the BigDye Terminator Cycle Sequencing Kit (Applied Biosystems). Sequences were deposited at GenBank and are available under the accession numbers xxxxxx-xxxxxx.

\subsubsection{Phylogenetic reconstructions}

Phylogenetic analyses were conducted on the basis of nuclear sequence data, which were newly generated in this study (see above), and with complete mitochondrial genome sequences, which were obtained from GenBank (Supplementary table 5.3). Both data sets included seven colobine (Colobus, Piliocolobus, Trachypithecus, Semnopithecus, Presbytis, Nasalis, Pygathrix), three cercopithecine (Macaca, Papio, Chlorocebus or Cercopithecus) and two hominid genera (Homo, Pan), while the latter two were used as outgroup taxa. Sequences were aligned with ClustalX (Thompson et al., 1994) and manually adjusted. After the manual removal of indel positions (758 bp), the concatenated nuclear alignment comprised 8,917 bp. In the mitochondrial data set, 895 ambiguous positions were removed with Gblocks (Castresana, 2000), resulting in an alignment with 15,905 bp in length. Phylogenetic trees were constructed with MP and NJ algorithms as implemented in PAUP 4.0b10 (Swofford, 2002) as well as with ML and Bayesian algorithms, using the programs GARLI 0.951 (Zwickl, 2006) and MrBayes 3.1.2 (Huelsenbeck and Ronquist, 2001; Ronquist and Huelsenbeck, 2003), respectively. Calculations were performed for each locus separately, for concatenated autosomal and $\mathrm{Y}$ chromosomal data sets and for a data set, in which all nuclear data were combined. However, mitochondrial and nuclear data were never combined. For MP analyses, all characters were treated as unordered and equally weighted throughout. A heuristic search was performed with the maximum number of trees set to 100 . NJ, ML and Bayesian trees were constructed with the respective best-fitting models as selected under the Akaike information criterion with MODELTEST 3.7 (Posada and Crandall, 1998) (see also supplementary table 5.1). Relative support of internal nodes was performed by bootstrap analyses with 10,000 (MP, NJ) or 500 replications (ML). In GARLI, only the model specifications settings were adjusted according to the respective data set, while all other settings were left at their default value. ML majority-rule consensus trees were calculated in PAUP. For Bayesian analyses, four Monte Carlo Markov Chains (MCMC) with the default temperature of 0.1 were used. Four repetitions were run for $10,000,000$ generations with tree and parameter sampling occurring every 100 generations. The first $25 \%$ of samples were discarded as burnin, leaving 75,001 trees per run. Posterior probabilities for each split were calculated from the posterior density of trees. 
To evaluate the reliability of the depicted relationships among colobines, alternative tree topologies were evaluated with the Kishino-Hasegawa (Kishino and Hasegawa, 1989) and Shimodaira-Hasegawa (Shimodaira and Hasegawa, 1999) tests with full optimization and 1,000 bootstrap replications in PAUP. Therefore, various hypothetical sister group relationships among Asian colobine lineages as well as a putative paraphyly of African colobines was tested.

\subsubsection{Divergence age estimation}

A Bayesian MCMC method, which employs a relaxed molecular clock approach, as implemented in the BEAST 1.4.8 package (Drummond and Rambaut, 2007), was applied to estimate divergence times. Calculations for the concatenated nuclear and mitochondrial data sets were performed separately and on the basis of a priori fixed tree topologies. For all estimations, a relaxed lognormal model of lineage variation, a Yule prior for branching rates and the optimal nucleotide substitution model as listed in supplementary table 5.1 were used. As calibration points we applied the split between Homo and Pan, which has been dated at 6-7 mya (Steiper and Young, 2006), the divergence between Papio and Macaca which is estimated at 6-8 mya (Steiper and Young, 2006), and the spilt between hominids and cercopithecids, which is estimated at 26.9-36.4 mya (Steiper and Young, 2006). Four replicates were run for 10 million generations with tree and parameter sampling occurring every 100 generations. The adequacy of a $10 \%$ burnin and convergence of all parameters were assessed by visual inspection of the trace of the parameters across generations using the software TRACER 1.4 (Rambaut and Drummond, 2007). Subsequently, the sampling distributions were combined (25\% burnin) using LogCombiner 1.4.8 and a consensus chronogram with node height distribution was generated and visualized with the software TreeAnnotator 1.4.8 and FigTree v1.1.2 (Rambaut, 2008).

\section{Acknowledgements}

We thank the zoos in Cologne, Dresden, Howletts, Leipzig, Munich, Nuremberg, Stuttgart and Wuppertal, and Linda Vigilant, Roland Plesker and Werner Schempp for providing samples. This study was financially supported by the German Primate Center. 


\section{Summary and Conclusion}

\subsection{Summary}

\subsubsection{New World monkeys}

Although already two SINE based studies, carried out by Singer et al. (2003) and Ray et al. (2005), are available, several important questions in New World monkey phylogeny remained. The reason therefore is that only few insertion events were analysed (Singer et al., 2003) or that a relative large number of genera were not tested (Ray et al., 2005). The first and most important issue in New World monkey phylogeny is the branching order among the three families Cebidae, Atelidae and Pitheciidae, which was not solved with significance in earlier studies. Associated with this issue is the question, whether the family Cebidae is monophyletic, more precisely whether Saimiri, Aotus and Cebus have to be included therein. Moreover, various contradicting phylogenetic affiliations among Saimiri, Aotus and Cebus were proposed by molecular and morphological studies, but never tested by SINE analyses. Furthermore, Ray et al. (2005) did not include Callimico, Leontopithecus, Cebus, Brachyteles, Cacajao and Chiropotes in their study. Hence, a complete phylogeny of Callithrichinae as well as Atelidae and Pitheciidae is still lacking.

This thesis includes all New World monkey genera combined with a relatively large dataset (128 SINE insertions were analysed), in contrast to earlier SINE based studies. In general, all relationships as depicted in earlier studies were confirmed and now a common origin of New World monkeys can be regarded as settled. Moreover, this thesis provides strong conclusive evidence for the unity of Pitheciidae and Atelidae and most importantly for a monophyletic origin of Cebidae, including the genera Saimiri, Aotus and Cebus. The monophyly of Atelidae is also confirmed, although Ray et al. (2005) provided already significant evidence for that. Hence, the diversification of New World monkeys into three different families Cebidae, Atelidae and Pitheciidae (Goodman et al., 1998; Opazo et al., 2006) is strongly supported. The branching order of these three families is now elucidated, with Pitheciidae as the most basal family, resulting in a grouping of Atelidae and Cebidae.

Within the Cebidae the phylogenetic positions of Saimiri, Aotus and Cebus could not be solved. Three independent SINE insertions were found, each of them showing different relationships among these three genera: either Saimiri and Cebus, Saimiri and Aotus or Aotus and Cebus cluster together. These contradicting results could be caused by incomplete lineage sorting of ancestral polymorphic 
insertions. This would be also supported by studies (e.g. Schrago, 2007) which estimated a relative short time period in which these three genera and Callithrichinae diverged from each other. Moreover, this assumption is further supported by a relatively large number of detected autapomorphic SINE insertions, at least in Saimiri and Aotus. Alltogether these ascertainments could explain why numerous molecular studies proposed different phylogenetic affiliations of those three genera within Cebidae and morphological studies even in the whole infraorder.

In contrast to the ambiguous relationships of Saimiri, Cebus and Aotus among cebids, this thesis provides clear evidence for the branching order among Callithrichinae genera. The monophyletic origin of all callithrichines was confirmed and is in agreement with other molecular studies. The positioning of Callimico within callitrichines was a challenging issue for a long time but at least molecular studies indicated a sister grouping to Callithrix. This sister-relationship is conclusively supported herein by seven SINE insertions. Moreover, this thesis shows evidence for a close affiliation of Leontopithecus, Callimico and Callithrix to the exclusion of Saguinus. Therefore, earlier classifications with Saguinus and Leontopithecus as sister genera can be rejected (e.g. Goodman et al., 1998).

Within the Atelidae, Alouatta could clearly be separated from Ateles, Lagothrix and Brachyteles, which supports molecular as well as morphological analyses (e.g. Ford, 1986; Goodman et al., 1998). The splitting of the family into the two subfamilies Atelinae and Alouatinae seems thus to be appropriate. Unfortunately no further SINE insertions could be found resolving the remaining relationships within Atelinae and Pitheciidae.

In the frame of this New World monkey study, we identified an Alu insertion, distinguishing between Saimiri sciureus and S. b. boliviensis. Squirrel monkeys are common in zoos and widely used in biomedical research. This molecular cladistic marker may help to improve captive breeding management, since an exact species identification based on morphological characteristics and other molecular markers is delicate. Moreover, the presence/absence pattern among the analyzed S. $b$. peruviensis specimen indicates that this study population, and therefore most of European captive animals of this subspecies, might have originated from a natural hybrid zone in the margins of the Ucayali River in the Peruvian Amazonia. This molecular marker can easily improve the breeding management of captive populations, which is of great interest for conservation, but also for biomedical research, since both species differ in critical biological parameters, as e.g. the susceptibility to diseases. 


\subsubsection{Colobine monkeys}

A comprehensive mobile element based phylogeny of colobine monkeys is still missing. Xing et al. (2005) performed a first attempt, which addressed Cercopithecidae phylogeny in general and therefore included just four (five) from actual ten different colobine genera. Crucial questions concerning a monophyletic origin of Asian and African colobines as well as the affiliations of the langurs Trachypithecus and Semnopithecus and the position of Presbytis within Asian colobines could not be answered. Moreover, the unity of the odd-nosed monkeys was merely indicated. Within this thesis these decisive long-standing questions could be definitely answered with surprising and unexpected results.

Monophyly of African and Asian colobines was not unquestioned. Although most morphological and molecular studies (e.g. Zhang and Ryder, 1998; Page et al., 1999; Ting, 2008) clearly proposed a monophyletic origin of Asian and African colobines, others (e.g. Groves, 1989; Peng et al., 1993; Jablonski, 1998) considered a paraphyly of Asian or a polyphyly of African colobines. This thesis strongly provides reliable evidence for paraphyly of African colobines and a monophyletic origin of Asian colobines. More precisely, this result includes three different Alu insertions, shared by all Asian colobines and the African genus Piliocolobus. However, to underpin or disclaim this finding mitochondrial and nuclear markers were incorporated as well. Mitochondrial data clearly supported a monophyletic origin of Asian and African colobines respectively, whereas nuclear data indicate paraphyly of African genera. Inadequate data, homoplasy or incomplete lineage sorting may have affected the tree topologies to some degree (Funk and Omland, 2003). However, these factors provide no sufficient explanation for the observed incongruences. Homoplasy and incomplete lineage sorting for Alu insertions is unlikely. Hence, it seems most likely that this pattern was caused by ancestral hybridizations, which is also supported by socioecological characteristics, morphology and distribution. In contrast to most other primate females, those of Piliocolobus tend to leave their natal groups (Newton and Dunbar, 1994). Furthermore, Colobus males are in average larger than Pilicolobus males and both genera occur over wide ranges in sympatry (Oates et al., 1994).

This thesis adduces further evidence for the monophyly of Asian colobines, whereas the evolution within Asian colobines seems to be indeed complex. Phylogenetic affiliations within Asian colobines and whether langurs and odd-nosed monkeys form monophyletic groups were major parts of debate. Concerning odd-nosed monkeys the question remains whether they represent more than an informal group as it was proposed 2001 by Groves. Not only sequence data clearly supported their monophyletic origin (Sterner et al., 2006), but also SINE data (Xing et al., 2005). This thesis supplies conclusive evidence for their monophyly and the relationships among them. Two Alu insertions 
identified Rhinopithecus as the most basal genus, followed by the separation of Pygathrix, whereas Nasalis and Simias form a sister relationship and diverged relative recently. These data are not shown and will be shortly implemented in another publication combined with comprehensive mitochondrial genome and nuclear sequence data (Roos et al., 2008, in prep).

Nuclear data and Alu insertions support a close relationship of Trachypithecus and Semnopithecus, and between Presbytis and the odd-nosed monkeys. In contrast, mitochondrial data result in an unresolved position of Semnopithecus relative to a Presbytis/Trachypithecus and an odd-nosed monkey clade. Positioning Presbytis among Asian colobines rely on Alu insertions. As in African colobines, ancestral hybridization seems to be the most plausible explanation for the incongruent gene trees. The close relationship of Semnopithecus and Trachypithecus is also supported by morphological characteristics. Moreover, hybridization events are in general possible due to partial sympatry (Oates et al., 1994). Accordingly, these incongruences among nuclear and mitochondrial gene trees indicate a complex evolutionary scenario, in which Semnopithecus males hybridized with Trachypithecus females leading to a fixation of the mitochondrial lineage in hybrids. By backcrossing with Semnopithecus males over a relative long time period (until $\sim 2.3 \pm 0.5$ mya), the progenitor of Trachypithecus enriched solely nuclear material of Semnopithecus, while the mitochondrial genome remained Trachypithecuslike.

By combining the obtained phylogenies and estimated divergence ages with paleoenvironmental data, some biogeographic suggestions can be provided. Colobines most likely originated in Africa, which is in agreement to Stewart and Disotell (1998). This is supported by the herein confirmed paraphyly, because a putative Asian origin would require two independent migrations into Africa. Furthermore, this is also supported by the fact that most cercopithecines (except Asian macaques) are solely distributed in Africa. The progenitor of Asian colobines most likely invaded Eurasia via an emerging landbridge connection between Africa and the Arabian Peninsula in the late Miocene, sometimes after the separation from Piliocolobus ( 10.4 \pm 1 mya) and before its diversification ( 8.1 \pm 1.2 mya). The Hengduan Mountains in the border region of today's Burma, India and China were proposed as a possible diversification hotspot for Asian colobines (Peng et al., 1993) which is supported by the fact that most of the larger Southeast-Asian rivers (Mekong, Salween, Yangtze) emerge there. Whether the ancestor of Asian colobines took a route north or south of the Himalaya from Africa to this hotspot is putative, although colobines from the later Miocene were found on the Tibetan plateau (Delson, 1994). After successive migration events, Trachypithecus and Semnopithecus came into secondary contact and exchanged genetic material until $\sim 2.3 \pm 0.5$ mya, most likely in the region of today's Bangladesh, Burma and the Northeast of India.

A further part of this thesis provides detailed insight into the genus affiliations of the species groups of Semnopithecus and Trachypithecus. According to our data, the genus Trachypithecus comprises three species groups (T. [obscurus], T. [cristatus] and T. [francoisi]), whereas two other species, 
previously also recognized as species of Trachypithecus (Trachypithecus johnii and Trachypithecus vetulus), should be included Semnopithecus. Moreover, the mitochondrial results support a diversification of the langurs from the Indian subcontinent into three species groups: one in North India comprising solely Semopithecus entellus populations, another in South India including T. johnii and S. entellus from South India, and finally a third group on Sri Lanka with T. vetulus and Sri Lankan S. entellus. Furthermore, the results show substantial discrepancies among the analysed gene trees for Trachypithecus pileatus and give therefore a hint for an ancient hybridization event between Semnopithecus and Trachypithecus. This hybridization hypothesis is also supported by some intermediate morphological characters (Groves, 2001) and the distribution of Trachypithecus pileatus, which is between Semnopithecus and other Trachypithecus species groups. In a recent study (Wangchuk et al., 2008) however, all tested T. pileatus cluster together with other Trachypithecus representatives, which contradicts with our (Osterholz et al. 2008) and other findings (Karanth et al., 2008). To explain these results, Wangchuk et al. (2008) proposed a hybrid origin for the individual analysed by Karanth et al. (2008). However, the individual analysed in our study is a museum specimen and was taken from the wild. Accordingly, an artificial hybrid origin can be excluded. A possible explanation for these findings might be that mitochondrial haplotypes of both Semnopithecus and Trachypithecus ancestors are still present in the T. pileatus population. To definetly solve this issue, further individuals and molecular markers have to be tested. Nevertheless, if the results in this thesis can be further supported in ongoing studies, this will underpin that hybridization plays a more important role in speciation mechanisms as previously thought, at least in colobines.

\subsection{Contributions to Primatology and Evolution}

This thesis resolves several highly disputed phylogenetic issues in primatology, especially in the evolutionary history of New World and colobine monkeys. Together with other studies, a SINE based phylogeny for the entire primate order is nearly complete. However, there are still some questions concerning the branching among Lemuriformes families, Galagidae genera or gibbon genera, which are not settled yet. A supervised diploma thesis (Kuska, 2008) was addressed to the remaining questions in strepsirrhine phylogeny. Unfortunately no further Alu insertions were detected, which elucidate remaining questions in Galagidae and Lemuriformes phylogeny, although former studies were generally confirmed. In the course of this thesis an attempt was made to resolve gibbon phylogeny, but without noteworthy results. Several solely genus specific insertion events were found by computational and experimental approaches, which is not surprising regarding the relative short time period in which the four genera diverged from each other (Roos and Geissmann, 2001). Some questions also remained in New World monkey phylogeny, like relationships within Atelidae and 
Pitheciidae, and the positions of Saimiri, Aotus and Cebus. Atelidae and Pitheciidae evolution could be resolved by further mobile element based analyses. To resolve relationships within both families an experimental approach would be more promising than a computational approach, because only few sequences are available so far. Furthermore, the Callithrix genome is lately available and could act as reference sequence for an improved oligonucleotide design. The positions of Saimiri, Aotus and Cebus seem to be more complex issues with important and decisive evolutionary implications. Many more efforts are required to solve respective relationships. Few insertions can be found in radiation-like diversification events because splitting times are too rapid for a fixation in a population. In this thesis contradicting results were solely found for Saimiri, Aotus and Cebus. It is worth mentioning that retroposons could also represent a strong indication for these types of diversification causing incomplete lineage sorting phenomena and contradicting results of different sequence-based topologies. Taking all disadvantages (e.g. homoplasy) of other systems into account it would be necessary to reconsider phylogenetic concepts, which are solely based on sequence data.

With this nearly completely resolved phylogeny of primates, a basis could be provided for other fields, like socioecology, forensic science or biomedical research. The Saimiri marker presented in Chapter 3 is a further example for the importance of retroposons in a valid identification of organisms. Retroposons provide an ideal alternative approach requiring a minimum of equipment and time. The identification is often the first step after receiving samples from unkown origin, the wild or zoos and hence informative retroposons are also important for conservation interests (e.g. illegal wildlife trade). Furthermore, they have proven to be useful for forensic purposes in quantifying limited DNA as well as identifying human geographic origins or gender (e.g. Hedges et al., 2003; Walker et al., 2003).

The results of this thesis underpin that retroposons are still one of the most promising tools in molecular cladistic, because they represent a reliable, nearly homoplasy free and unbiased system. The only pitfall in analysing retroposons remains the possibility of differential lineage sorting on weak supported branches.

An important achievement of this thesis is the finding that - at least in primates - hybridization occurs among genera and hence probably plays a decisive role in diversification. The data provide incentives for modifications of main evolutionary principles. Several studies proposed similar hypotheses for several years. This thesis impressively demonstrates that retroposons can contribute to unequivocally reveal phylogenetic relationships, which are otherwise not resolvable by pure nuclear sequence data. Comparing them with relationships obtained from mitochondrial sequence data can give a hint to such hybridization events. Colobine monkeys and especially their diversification in Southeast Asia form the most prominent example. Consequently, we have to reconsider concepts, where hybridization leads principally to a limited diversification, reproductive isolation or lower fitness, like it was proposed by Darwin (1859) or Mayr (1963). 


\section{Bibliography}

Alberts, S. C. and Altmann, J. 2001. Immigration and hybridization patterns of yellow and anubis baboons in and around Amboseli, Kenya. Am. J. Primatol. 53:139-154.

Andrews, T. D., Jermiin, L. S. and Easteal, S. 1998. Accelerated evolution of cytochrome b in simian primates: adaptive evolution in concert with other mitochondrial proteins? J. Mol. Evol. 47:249-257.

Andriaholinirina, N., Fausser, J. L., Roos, C. et al. 2006. Molecular phylogeny and taxonomic revision of the sportive lemurs (Lepilemur, Primates). BMC Evol. Biol. 6:17.

Arnason, U., Adegoke, J. A., Bodin, K., Born, E. W., Esa, Y. B., Gullberg, A., Nilsson, M., Short, R. V., Xu, X. and Janke, A. 2002. Mammalian mitogenomic relationships and the root of the eutherian tree. Proc. Natl. Acad. Sci. U S A 99:8151-8156.

Arnold, M. L. and Meyer, A. 2006. Natural hybridization in primates: one evolutionary mechanism. Zoology 109:261-276.

Avise, J. C. 2000. Phylogeography - The History and Formation of Species. Harvard University Press, Cambridge, MA.

Barton, N. H. 2001. The role of hybridization in evolution. Mol. Ecol. 10:551-568.

Batzer, M. A. and Deininger, P. L. 2002. Alu repeats and human genomic diversity. Nat. Rev. Genet. 3:370-379.

Batzer, M. A., Deininger, P. L., Hellmann-Blumberg, U., Jurka, J., Labuda, D., Rubin, C. M., Schmid, C. W., Zietkiewicz, E. and Zuckerkandl, E. 1996. Standardized nomenclature for Alu repeats. J. Mol. Evol. 42:36.

Bennett, E. L. and Davies, A. G. 1994. The ecology of Asian colobines. Pp. 129-171 in A. G. Davies, and J. F. Oates, eds. Colobine Monkeys: Their Ecology, Behaviour, and Evolution. Cambridge University Press, Cambridge.

Berthier, P., Excoffier, L. and Ruedi, M. 2006. Recurrent replacement of mtDNA and cryptic hybridization between two sibling bat species Myotis myotis and Myotis blythii. Proc. Biol. Sci. 273:3101-3109.

Bicca-Marques, J. C., Prates, H. M., de Aguiar, F. R. and Jones, C. B. 2008. Survey of Alouatta caraya, the blackand-gold howler monkey, and Alouatta guariba clamitans, the brown howler monkey, in a contact zone, State of Rio Grande do Sul, Brazil: evidence for hybridization. Primates.

Bigoni, F., Houck, M. L., Ryder, O. A., Wienberg, J. and Stanyon, R. 2004. Chromosomal painting shows that Pygathrix nemaeus has the most absal karyotype among Asian colobines. Int. J. Primatol. 25:679-688.

Bigoni, F., Stanyon, R., Wimmer, R. and Schempp, W. 2003. Chromosome painting shows that the proboscis monkey (Nasalis larvatus) has a derived karyotype and is phylogenetically nested within Asian Colobines. Am. J. Primatol. 60:85-93.

Boinski, S. and Cropp, S. 1998. Disparate data sets resolve squirrel monkey (Saimiri) taxonomy: implications for behavioral ecology and biomedical usage. Int. J. Primatol. 20:237-256.

Boinski, S. and Newman, J. D. 1988. Preliminary observations of squirrel monkey (Saimiri oerstedii) vocalizations in Costa Rica. Am. J. Primatol. 14:329-343.

Boubli, J. P., da Silva, M. N. F., Amado, M. V., Hrbek, T., Pontual, F. B. and Farias, I. P. 2008. A taxonomic reassessment of Cacajao melanocephalus Humboldt (1811), with the description of two new species. Int. J. Primatol. 29:723-741.

Brandon-Jones, D. 1995. A revision of the Asian pied leaf monkeys (Mammalia: Cercopithecidae: Superspecies Semnopithecus auratus), with a description of a new subspecies. The Raffles Bulletin of Zoology 43:3-43.

Brandon-Jones, D. 1984. Colobus and leaf monkeys. Pp. 398-403 in D. MacDonald, ed. The Encylopedia of Mammals. Allen \& Unwin, London.

Brandon-Jones, D., Eudey, A. A., Geissmann, T., Groves, C. P., Melnick, D. J., Morales, J. C., Shekelle, M. and Stewart, C. B. 2004. Asian Primate Classification. Int. J. Primatol. 25:97-164. 
Britten, R. J. and Kohne, D. E. 1968. Repeated sequences in DNA. Hundreds of thousands of copies of DNA sequences have been incorporated into the genomes of higher organisms. Science 161:529-540.

Brown, W. M., Prager, E. M., Wang, A. and Wilson, A. C. 1982. Mitochondrial DNA sequences of primates: tempo and mode of evolution. J. Mol. Evol. 18:225-239.

Canavez, F. C., Moreira, M. A., Ladasky, J. J., Pissinatti, A., Parham, P. and Seuanez, H. N. 1999. Molecular phylogeny of new world primates (Platyrrhini) based on beta2-microglobulin DNA sequences. Mol. Phylogenet. Evol. 12:74-82.

Cann, R. L., Stoneking, M. and Wilson, A. C. 1987. Mitochondrial DNA and human evolution. Nature 325:31-36.

Castresana, J. 2000. Selection of conserved blocks from multiple alignments for their use in phylogenetic analysis. Mol. Biol. Evol. 17:540-552.

Chakraborty, D., Ramakrishnan, U., Panor, J., Mishra, C. and Sinha, A. 2007. Phylogenetic relationships and morphometric affinities of the Arunachal macaque Macaca munzala, a newly described primate from Arunachal Pradesh, northeastern India. Mol. Phylogenet. Evol. 44:838-849.

Chaves, R., Sampaio, I., Schneider, M. P., Schneider, H., Page, S. L. and Goodman, M. 1999. The place of Callimico goeldii in the Callitrichine phylogenetic tree: evidence from von Willebrand factor gene intron II sequences. Mol. Phylogenet. Evol. 13:392-404.

Chivers, D. J. and Hladik, C. M. 1980. Morphology of the gastrointestinal tract in primates: comparisons with other mammals in relation to diet. J. Morphol. 166:337-386.

Collins, A. C. 2004. Atelinae phylogenetic relationships: the trichotomy revived? Am. J. Phys. Anthropol. 124:285296.

Collura, R. V., Auerbach, M. R. and Stewart, C. B. 1996. A quick, direct method that can differentiate expressed mitochondrial genes from their nuclear pseudogenes. Curr. Biol. 6:1337-1339.

Collura, R. V. and Stewart, C. B. 1995. Insertions and duplications of mtDNA in the nuclear genomes of Old World monkeys and hominoids. Nature 378:485-489.

Cortes-Ortiz, L., Duda, T. F., Jr., Canales-Espinosa, D., Garcia-Orduna, F., Rodriguez-Luna, E. and Bermingham, E. 2007. Hybridization in large-bodied New World primates. Genetics 176:2421-2425.

Costello, R. K., Dickinson, C., Rosenberger, A. L., Boinski, S. and Szalay, F. S. 1993. A multidisciplinary approach to squirrel monkey (genus Saimiri) species taxonomy in W. Kimbel, and L. Martin, eds. Species, Species Concepts, and Primate Evolution. Plenum Press, New York.

Cropp, S. and Boinski, S. 2000. The Central American squirrel monkey (Saimiri oerstedii): introduced hybrid or endemic species? Mol. Phylogenet. Evol. 16:350-365.

Darwin, C. 1859. On the Origin of Species by Means of Natural Selection or the Preservation of Favoured Races in the Struggle of Life. John Murray, London.

Davenport, T. R., Stanley, W. T., Sargis, E. J., De Luca, D. W., Mpunga, N. E., Machaga, S. J. and Olson, L. E. 2006. A new genus of African monkey, Rungwecebus: morphology, ecology, and molecular phylogenetics. Science 312:1378-1381.

Davies, A. G. and Oates, J. F. 1994. Colobine monkeys: their ecology, behaviour, and evolution. Cambridge University Press, Cambridge.

de Jong, W. W., van Dijk, M. A., Poux, C., Kappe, G., van Rheede, T. and Madsen, O. 2003. Indels in proteincoding sequences of Euarchontoglires constrain the rooting of the eutherian tree. Mol. Phylogenet. Evol. 28:328-340.

de Lima, M. M., Sampaio, I., Vieira Rdos, S. and Schneider, H. 2007. Spider monkey, Muriqui and Woolly monkey relationships revisited. Primates 48:55-63.

Deininger, P. L. and Batzer, M. A. 1993. Evolution of retroposons. Evol. Biol. 27:157-196.

Deininger, P. L., Batzer, M. A., Hutchison, C. A., 3rd and Edgell, M. H. 1992. Master genes in mammalian repetitive DNA amplification. Trends Genet. 8:307-311.

Delson, E. 1975. Evolutionary history of the Cercopithecidae. Contrib. Primatol. 5:167-217. 
Delson, E. 1994. Evolutionary history of the colobine monkeys in paleoenvironmental perspective. Pp. 45-74 in A. G. Davies, and J. F. Oates, eds. Colobine Monkeys: Their Ecology, Behaviour, and Evolution. Cambridge University Press, Cambridge.

Delson, E. 1992. Evolution of old world monkeys. Pp. 217-222 in J. S. Johns, R. D. Martin, D. Pilbeam, and S. Bunney, eds. The Cambridge Encyclopedia of Human Evolution. Cambridge University Press, Cambridge.

Diatchenko, L., Lau, Y. F., Campbell, A. P. et al. 1996. Suppression subtractive hybridization: a method for generating differentially regulated or tissue-specific cDNA probes and libraries. Proc. Natl. Acad. Sci. U S A 93:6025-6030.

Disotell, T. R. 1994. Generic level relationships of the Papionini (Cercopithecoidea). Am. J. Phys. Anthropol. 94:47-57.

Drummond, A. J. and Rambaut, A. 2007. BEAST: Bayesian evolutionary analysis by sampling trees. BMC Evol. Biol. 7:214.

Elliot, D. G. 1913. A Review of the Primates. American Museum of Natural History, New York.

Evans, B. J., Supriatna, J., Andayani, N. and Melnick, D. J. 2003. Diversification of Sulawesi macaque monkeys: decoupled evolution of mitochondrial and autosomal DNA. Evolution 57:1931-1946.

Farris, S., Albert, V., Källersjö, M., Lipscomb, D. and Kluge, A. 1996. Parsimony Jackknifing Outperforms Neighbor-Joining. Cladistics 12:99-124.

Felsenstein, J. 1978. Cases in Which Parsimony and Compatibility Methods Will Be Positively Misleading. Syst. Zool. 27:401-410.

Felsenstein, J. 1981. Evolutionary trees from DNA sequences: a maximum likelihood approach. J. Mol. Evol. 17:368-376.

Felsenstein, J. 1985. Confidence-Limits on Phylogenies - an Approach Using the Bootstrap. Evolution 39:783-791.

Feng, Q., Moran, J. V., Kazazian, H. H., Jr. and Boeke, J. D. 1996. Human L1 retrotransposon encodes a conserved endonuclease required for retrotransposition. Cell 87:905-916.

Fleagle, J. F. 1988. Primate Adaptation and Evolution. Academic Press, New York.

Flynn, J. J. and Nedbal, M. A. 1998. Phylogeny of the Carnivora (Mammalia): congruence vs incompatibility among multiple data sets. Mol. Phylogenet. Evol. 9:414-426.

Ford, S. M. 1986. Systematics of the New World monkeys. Pp. 73-135 in D. R. Swindler, and J. Erwin, eds. Comparative Primate Biology, Vol. 1: Systematics, Evolution, and Anatomy. A. R. Liss, New York.

Funk, D. J. and Omland, K. E. 2003. Species-Level Paraphyly and Polyphyly: Frequency, Causes, and Consequences, with Insights from Animal Mitochondrial DNA. Annu. Rev. Ecol. Evol. Syst. 34:397-423.

Geissmann, T. 2003. Vergleichende Primatologie. Springer, Berlin/Heidelberg.

Geissmann, T., Groves, C. P. and Roos, C. 2004. The Tenasserim Lutung, Trachypithecus barbei (Blyth, 1847) (Primates: Cercopithecidae): Description, of a live specimen, and a reassessment of phylogenetic affinities, taxonomic history, and distribution. Contrib. Zool. 73:271-282.

Gibbs, R. A., Rogers, J., Katze, M. G. et al. 2007. Evolutionary and biomedical insights from the rhesus macaque genome. Science 316:222-234.

Goodman, M., Porter, C. A., Czelusniak, J., Page, S. L., Schneider, H., Shoshani, J., Gunnell, G. and Groves, C. P. 1998. Toward a phylogenetic classification of Primates based on DNA evidence complemented by fossil evidence. Mol. Phylogenet. Evol. 9:585-598.

Goodman, M., Tagle, D. A., Fitch, D. H., Bailey, W., Czelusniak, J., Koop, B. F., Benson, P. and Slightom, J. L. 1990. Primate evolution at the DNA level and a classification of hominoids. J. Mol. Evol. 30:260-266.

Goossens, B., Chikhi, L., Utami, S. S., De Ruiter, J. R. and Bruford, M. W. 2000. A multi-samples, multi-extracts approach for microsatellite analysis of faecal samples in an aboreal ape. Conserv. Genet. 1:157-162.

Grant, P. R. and Grant, B. R. 2002. Unpredictable evolution in a 30-year study of Darwin's finches. Science 296:707-711. 
Grant, P. R., Grant, B. R. and Petren, K. 2005. Hybridization in the recent past. Am. Nat. 166:56-67.

Groves, C. P. 2001. Primate Taxonomy. Pages Smithsonian Press, Washington, DC.

Groves, C. P. 1989. A Theory of Human and Primate Evolution. Clarendon Press, Oxford.

Groves, C. P. 1970. The forgotten leaf-eaters and the phylogeny of Colobinae. Pp. 555-586 in J. R. Napier, and P. H. Napier, eds. Old World Monkeys: Ecology, Systematics, and Behaviour. Academic Press, New York.

Grubb, P., Butynski, T. M., Oates, J. F., Bearder, S. K., Disotell, T. R., Groves, C. P. and Struhsaker, T. T. 2003. Assessment of the diversity of African primates. Int. J. Primatol. 24:1301-1357.

Hall, T. A. 1999. BioEdit: a user-friendly biological sequence alignment editor and analysis program for Windows 95/98/NT. Nucleic Acids Res. 41:95-98.

Harada, M. L., Schneider, H., Schneider, M. P., Sampaio, I., Czelusniak, J. and Goodman, M. 1995. DNA evidence on the phylogenetic systematics of New World monkeys: support for the sister-grouping of Cebus and Saimiri from two unlinked nuclear genes. Mol. Phylogenet. Evol. 4:331-349.

Hedges, D. J., Walker, J. A., Callinan, P. A., Shewale, J. G., Sinha, S. K. and Batzer, M. A. 2003. Mobile elementbased assay for human gender determination. Anal. Biochem. 312:77-79.

Hellborg, L. and Ellegren, H. 2003. Y chromosome conserved anchored tagged sequences (YCATS) for the analysis of mammalian male-specific DNA. Mol. Ecol. 12:283-291.

Hennig, W. 1950. Grundzüge einer Theorie der phylogenetischen Systematik. Deutscher Zentralverlag, Berlin.

Herke, S. W., Xing, J., Ray, D. A., Zimmerman, J. W., Cordaux, R. and Batzer, M. A. 2007. A SINE-based dichotomous key for primate identification. Gene 390:39-51.

Hershkovitz, P. 1984. Taxonomy of the squirrel monkeys, genus Saimiri (Cebidae, Platyrrhini): A preliminary report with description of a hitherto unnamed form. Am. J. Primatol. 6:257-281.

Hershkovitz, P. 1977. Living New World monkeys, part 1 (Platyrrhini) with an introduction to primates. Chicago University Press, Chicago.

Hewitt, G. M. 2001. Speciation, hybrid zones and phylogeography - or seeing genes in space and time. Mol. Ecol. 10:537-549.

Hill, W. C. O. 1934. A monography on the purple-faced leaf-monkeys (Pithecus vetulus). Ceylon J. Sci. 9:23-88.

Hill, W. C. O. 1960. Primates, Comparative Anatomy and Taxonomy. IV. Cebidae, Part A. Interscience, New York.

Hofer, H. O. 1976. Preliminary study of the comparative anatomy of the external nose of South America monkeys. Folia Primatol. 25:193-214.

Horovitz, I. and Meyer, A. 1995. Systematics of New World monkeys (Platyrrhini, Primates) based on 16S mitochondrial DNA sequences: a comparative analysis of different weighting methods in cladistic analysis. Mol. Phylogenet. Evol. 4:448-456.

Horovitz, I., Zardoya, R. and Meyer, A. 1998. Platyrrhine systematics: a simultaneous analysis of molecular and morphological data. Am. J. Phys. Anthropol. 106:261-281.

Horvath, J. E., Weisrock, D. W., Embry, S. L., Fiorentino, I., Balhoff, J. P., Kappeler, P., Wray, G. A., Willard, H. F. and Yoder, A. D. 2008. Development and application of a phylogenomic toolkit: resolving the evolutionary history of Madagascar's lemurs. Genome Res. 18:489-499.

Huelsenbeck, J. P. and Ronquist, F. 2001. MRBAYES: Bayesian inference of phylogenetic trees. Bioinformatics 17:754-755.

IUCN. 2007. 2007 Red List of Endangered Animals. IUCN, Gland, Switzerland.

Jablonski, N. G. 1998. The Evolution of the Doucs and Snub-nosed monkeys and the question of the phyletic unity of the odd-nosed colobines. Pp. 13-52 in N. G. Jablonski, ed. Natural History of the Doucs and Snub-nosed monkeys. World Scientific Publishing Company, New Jersey.

Jolly, C. J., Woolley-Barker, T., Beyene, S., Disotell, T. R. and Phillips-Conroy, J. E. 1997. Intergeneric hybrid baboons. Int. J. Primatol. 18:597-627. 
Jones, T., Ehardt, C. L., Butynski, T. M., Davenport, T. R., Mpunga, N. E., Machaga, S. J. and De Luca, D. W. 2005. The highland mangabey Lophocebus kipunji: a new species of African monkey. Science 308:11611164.

Jorgensen, S. and Mauricio, R. 2005. Hybridization as a source of evolutionary novelty: leaf shape in a Hawaiian composite. Genetica 123:171-179.

Jurka, J. 1997. Sequence patterns indicate an enzymatic involvement in integration of mammalian retroposons. Proc. Natl. Acad. Sci. U S A 94:1872-1877.

Jurka, J., Kapitonov, V. V., Pavlicek, A., Klonowski, P., Kohany, O. and Walichiewicz, J. 2005. Repbase Update, a database of eukaryotic repetitive elements. Cytogenet. Genome Res. 110:462-467.

Kajikawa, M. and Okada, N. 2002. LINEs mobilize SINEs in the eel through a shared 3' sequence. Cell 111:433444.

Kappeler, P. M., Rasoloarison, R. M., Razafimanantsoa, L., Walter, L. and Roos, C. 2005. Morphology, behaviour and molecular evolution of giant mouse lemurs (Mirza spp.) gray, 1970, with description of a new species. Primate Report 71:3-26.

Karanth, K. P., Delefosse, T., Rakotosamimanana, B., Parsons, T. J. and Yoder, A. D. 2005. Ancient DNA from giant extinct lemurs confirms single origin of Malagasy primates. Proc. Natl. Acad. Sci. U S A 102:50905095.

Karanth, K. P., Singh, L., Collura, R. V. and Stewart, C. B. 2008. Molecular phylogeny and biogeography of langurs and leaf monkeys of South Asia (Primates: Colobinae). Mol. Phylogenet. Evol. 46:683-694.

Katoh, K., Kuma, K., Toh, H. and Miyata, T. 2005. MAFFT version 5: improvement in accuracy of multiple sequence alignment. Nucleic Acids Res. 33:511-518.

Kay, R. 1990. The phyletic relationships of extant and fossil Pitheciinae (Platyrrhini, Anthropoidea). Pp. 175-208 in J. F. Fleagle, and A. L. Rosenberger, eds. The Platyrrhine Fossil Record. Academic Press, New York.

Kazazian, H. H., Jr. 2004. Mobile elements: drivers of genome evolution. Science 303:1626-1632.

Kazazian, H. H., Jr. and Moran, J. V. 1998. The impact of L1 retrotransposons on the human genome. Nat. Genet. 19:19-24.

Kent, W. J., Sugnet, C. W., Furey, T. S., Roskin, K. M., Pringle, T. H., Zahler, A. M. and Haussler, D. 2002. The human genome browser at UCSC. Genome Res. 12:996-1006.

Kishino, H. and Hasegawa, M. 1989. Evaluation of the maximum likelihood estimate of the evolutionary tree topologies from DNA sequence data, and the branching order in hominoidea. J. Mol. Evol. 29:170-179.

Koblmüller, S., Duftner, N., Sefc, K. M., Aibara, M., Stipacek, M., Blanc, M., Egger, B. and Sturmbauer, C. 2007. Reticulate phylogeny of gastropod-shell-breeding cichlids from Lake Tanganyika--the result of repeated introgressive hybridization. BMC Evol. Biol. 7:7.

Kriegs, J. O., Churakov, G., Kiefmann, M., Jordan, U., Brosius, J. and Schmitz, J. 2006. Retroposed elements as archives for the evolutionary history of placental mammals. PLoS Biol. 4:e91.

Kriegs, J. O., Matzke, A., Churakov, G., Kuritzin, A., Mayr, G., Brosius, J. and Schmitz, J. 2007. Waves of genomic hitchhikers shed light on the evolution of gamebirds (Aves: Galliformes). BMC Evol. Biol. 7:190.

Kuhn, H.-J. 1967. Zur Systematik der Cercopithecidae. Pp. 25-46 in D. Starck, R. Schneider, and H.-J. Kuhn, eds. Neue Ergebnisse der Primatologie. Gustav Fischer, Stuttgart.

Kuryshev, V. Y., Skryabin, B. V., Kremerskothen, J., Jurka, J. and Brosius, J. 2001. Birth of a gene: locus of neuronal BC200 snmRNA in three prosimians and human BC200 pseudogenes as archives of change in the Anthropoidea lineage. J. Mol. Biol. 309:1049-1066.

Kuska, B. 2008. Molekulare Phylogenie der Strepsirrhini. Diploma thesis, University of Göttingen.

Lander, E. S., Linton, L. M. and IHGS, C. 2001. Initial sequencing and analysis of the human genome. Nature 409:860-921.

Lavergne, A., Catzeflis, F., Lacote, S., Barnaud, A., Bordier, M., Mercereau-Puijalon, O. and Contamin, H. 2003. Genetic analysis of the Saimiri breeding colony of the Pasteur Institute (French Guiana): development of 
a molecular typing method using a combination of nuclear and mitochondrial DNA markers. J. Med. Primatol. 32:330-340.

Li, W. H., Gu, Z., Wang, H. and Nekrutenko, A. 2001. Evolutionary analyses of the human genome. Nature 409:847-849.

Linnaeus, C. N. 1758. Systema Naturae Per Regna Tria Naturae, Secundum Classes, Ordines, Genera, Species, Cum Characteribus Differentiis, Synonymis, Locis.

Lönnerg, E. 1940. Notes on some members of the genus Saimiri. Ark. Zool. 32:1-18.

Luan, D. D., Korman, M. H., Jakubczak, J. L. and Eickbush, T. H. 1993. Reverse transcription of R2Bm RNA is primed by a nick at the chromosomal target site: a mechanism for non-LTR retrotransposition. Cell 72:595-605.

Luckett, W. P. 1980. Monophyletic or diphyletic origins of Anthropoidea and Hytricognathi: evidence of the fetal membranes. Pp. 347-368 in R. L. Ciochon, and A. B. Chiarelli, eds. Evolutionary Biology of the New World Monkeys and Continental Drift. Plenum Press, New York.

Mamedov, I. Z., Arzumanyan, E. S., Amosova, A. L., Lebedev, Y. B. and Sverdlov, E. D. 2005. Whole-genome experimental identification of insertion/deletion polymorphisms of interspersed repeats by a new general approach. Nucleic Acids Res. 33:e16.

Martin, R. D. 1990. Primate Origins and Evolution. A Phylogenetic Reconstruction. Chapman \& Hall, London.

Matveev, V., Nishihara, H. and Okada, N. 2007. Novel SINE families from salmons validate Parahucho (Salmonidae) as a distinct genus and give evidence that SINEs can incorporate LINE-related 3'-tails of other SINEs. Mol. Biol. Evol. 24:1656-1666.

Mayr, E. 1942. Systematics and the Origin of Species. Columbia University Press, New York.

Mayr, E. 1963. Animal Species and Evolution. Belknap Press, Cambridge, MA.

McCracken, K. and Sorenson, M. 2005. Is homoplasy or lineage sorting the source of incongruent mtdna and nuclear gene trees in the stiff-tailed ducks (Nomonyx-Oxyura)? Syst. Biol. 54:35-55.

McDonald, D. B., Parchman, T. L., Bower, M. R., Hubert, W. A. and Rahel, F. J. 2008. An introduced and a native vertebrate hybridize to form a genetic bridge to a second native species. Proc. Natl. Acad. Sci. U S A 105:10837-10842.

Meijaard, E. and Groves, C. P. 2006. The geography of mammals and rivers in mainland Southeast Asia. Pp. 305329 in S. M. Lehman, and J. G. Fleagle, eds. Primate Biogeography: Progress and Prospects. Springer, New York.

Messier, W. and Stewart, C. B. 1997. Episodic adaptive evolution of primate lysozymes. Nature 385:151-154.

Mittermeier, R. A., Konstant, W. R., Hawkins, F. et al. 2006. Lemurs of Madagascar. Conservation International, Washington, DC.

Mittermeier, R. A., Konstant, W. R. and Mast, R. B. 1994. Use of neotropical and Malagasy primate species in biomedical research. Am. J. Primatol. 34:73-80.

Mivart, S. G. J. 1873. On Lepilemur and Cheirogaleus and on the zoological rank of the Lemuroidea. Proc. Zool. Soc. Lond. 1873:484-510.

Moore, C. M., Harris, C. P. and Abee, C. R. 1990. Distribution of chromosomal polymorphisms in three subspecies of squirrel monkeys (genus Saimiri). Cytogenet. Cell. Genet. 53:118-122.

Mourier, T., Hansen, A. J., Willerslev, E. and Arctander, P. 2001. The Human Genome Project reveals a continuous transfer of large mitochondrial fragments to the nucleus. Mol. Biol. Evol. 18:1833-1837.

Murata, S., Takasaki, N., Saitoh, M. and Okada, N. 1993. Determination of the phylogenetic relationships among Pacific salmonids by using short interspersed elements (SINEs) as temporal landmarks of evolution. Proc. Natl. Acad. Sci. U S A 90:6995-6999.

Murphy, W. J., Eizirik, E., Johnson, W. E., Zhang, Y. P., Ryder, O. A. and O'Brien, S. J. 2001a. Molecular phylogenetics and the origins of placental mammals. Nature 409:614-618.

Murphy, W. J., Eizirik, E., O'Brien, S. J. et al. 2001b. Resolution of the early placental mammal radiation using Bayesian phylogenetics. Science 294:2348-2351. 
Nadler, T., Walter, L. and Roos, C. 2005. Molecular evolution, systematics and distribution of the taxa within the silvered langur species group (Trachypithecus [cristatus]) in Southeast Asia. Zoologischer Garten (NF) 75:238-247.

Napier, J. R. 1970. Paleoecology and catarrhine evolution. Pp. 53-95 in J. R. Napier, and P. H. Napier, eds. Old World Monkeys: Ecology, Systematics, and Behaviour. Academic Press, New York.

Napier, J. R. and Napier, P. H. 1967. A Handbook of Living Primates. Academic Press, London.

Newton, P. N. and Dunbar, R. I. M. 1994. Colobine monkey society. Pp. 311-346 in A. G. Davies, and J. F. Oates, eds. Colobine Monkeys: Their Ecology, Behaviour and Evolution. Cambridge University Press, Cambridge.

Nikaido, M., Rooney, A. P. and Okada, N. 1999. Phylogenetic relationships among cetartiodactyls based on insertions of short and long interpersed elements: hippopotamuses are the closest extant relatives of whales. Proc. Natl. Acad. Sci. U S A 96:10261-10266.

Nishihara, H., Satta, Y., Nikaido, M., Thewissen, J. G., Stanhope, M. J. and Okada, N. 2005. A retroposon analysis of Afrotherian phylogeny. Mol. Biol. Evol. 22:1823-1833.

Nolte, A. W., Freyhof, J. and Tautz, D. 2006. When invaders meet locally adapted types: rapid moulding of hybrid zones between sculpins (Cottus, Pisces) in the Rhine system. Mol. Ecol. 15:1983-1993.

Nsubuga, A. M., Robbins, M. M., Roeder, A. D., Morin, P. A., Boesch, C. and Vigilant, L. 2004. Factors affecting the amount of genomic DNA extracted from ape faeces and the identification of an improved sample storage method. Mol. Ecol. 13:2089-2094.

Oates, J. F., Davies, A. G. and Delson, E. 1994. The diversity of living colobines. Pp. 45-73 in A. G. Davies, and J. F. Oates, eds. Colobine Monkeys: Their Ecology, Behaviour and Evolution. Cambridge University Press, Cambridge.

Okada, N. 1991. SINEs. Curr. Opin. Genet. Dev. 1:498-504.

Opazo, J. C., Wildman, D. E., Prychitko, T., Johnson, R. M. and Goodman, M. 2006. Phylogenetic relationships and divergence times among New World monkeys (Platyrrhini, Primates). Mol. Phylogenet. Evol. 40:274-280.

Osterholz, M., Walter, L. and Roos, C. 2008. Phylogenetic position of the langur genera Semnopithecus and Trachypithecus among Asian colobines, and genus affiliations of their species groups. BMC Evol. Biol. 8:58.

Pace, J. K., 2nd and Feschotte, C. 2007. The evolutionary history of human DNA transposons: evidence for intense activity in the primate lineage. Genome Res. 17:422-432.

Page, S. L., Chiu, C. and Goodman, M. 1999. Molecular phylogeny of Old World monkeys (Cercopithecidae) as inferred from gamma-globin DNA sequences. Mol. Phylogenet. Evol. 13:348-359.

Page, S. L. and Goodman, M. 2001. Catarrhine phylogeny: noncoding DNA evidence for a diphyletic origin of the mangabeys and for a human-chimpanzee clade. Mol. Phylogenet. Evol. 18:14-25.

Pastorini, J., Forstner, M. R., Martin, R. D. and Melnick, D. J. 1998. A reexamination of the phylogenetic position of Callimico (primates) incorporating new mitochondrial DNA sequence data. J. Mol. Evol. 47:32-41.

Patterson, N., Richter, D. J., Gnerre, S., Lander, E. S. and Reich, D. 2006. Genetic evidence for complex speciation of humans and chimpanzees. Nature 441:1103-1108.

Peng, Y. Z., Pan, R. L. and Jablonski, N. G. 1993. Classification and evolution of Asian colobines. Folia Primatol. 60:106-117.

Pidancier, N., Jordan, S., Luikart, G. and Taberlet, P. 2006. Evolutionary history of the genus Capra (Mammalia, Artiodactyla): discordance between mitochondrial DNA and Y-chromosome phylogenies. Mol. Phylogenet. Evol. 40:739-749.

Piskurek, O., Austin, C. C. and Okada, N. 2006. Sauria SINEs: Novel short interspersed retroposable elements that are widespread in reptile genomes. J. Mol. Evol. 62:630-644.

Pocock, R. I. 1936. The external characters of a female red colobus monkey (Procolobus badius waldroni). Proceedings of the Zoological Society of London (1935):939-944. 
Pocock, R. I. 1935. The monkeys of the genera Pithecus (or Presbytis) and Pygathrix found to the east of the Bay of Bengal. Proceedings of the Zoological Society of London (1934):895-961.

Pocock, R. I. 1939. The Fauna of British India, Including Ceylon and Burma: Mammals. 1. Primates and Carnivores (in part), Families Felidae and Viverridae. Taylor \& Francis, London.

Porter, C. A., Czelusniak, J., Schneider, H., Schneider, M. P., Sampaio, I. and Goodman, M. 1997. Sequences of the primate epsilon-globin gene: implications for systematics of the marmosets and other New World primates. Gene 205:59-71.

Porter, C. A., Sampaio, I., Schneider, H., Schneider, M. P., Czelusniak, J. and Goodman, M. 1995. Evidence on primate phylogeny from epsilon-globin gene sequences and flanking regions. J. Mol. Evol. 40:30-55.

Posada, D. and Crandall, K. A. 1998. MODELTEST: testing the model of DNA substitution. Bioinformatics 14:817-818.

Prychitko, T., Johnson, R. M., Wildman, D. E., Gumucio, D. and Goodman, M. 2005. The phylogenetic history of New World monkey beta globin reveals a platyrrhine beta to delta gene conversion in the atelid ancestry. Mol. Phylogenet. Evol. 35:225-234.

Raaum, R. L., Sterner, K. N., Noviello, C. M., Stewart, C. B. and Disotell, T. R. 2005. Catarrhine primate divergence dates estimated from complete mitochondrial genomes: concordance with fossil and nuclear DNA evidence. J. Hum. Evol. 48:237-257.

Rambaut, A. 2008. FigTree v.1.1.2. Available from http://tree.bio.ed.ac.uk/software/figtree.

Rambaut, A. and Drummond, A. J. 2007. Tracer v1.4. Available from http://beast.bio.ed.ac.uk/Tracer.

Ray, D. A. and Batzer, M. A. 2005. Tracking Alu evolution in New World primates. BMC Evol. Biol. 5:51.

Ray, D. A., Xing, J., Hedges, D. J. et al. 2005. Alu insertion loci and platyrrhine primate phylogeny. Mol. Phylogenet. Evol. 35:117-126.

Reichenbach, H. G. L. 1862. Die vollständige Naturgeschichte der Affen. Die vollständige Naturgeschichte des In- und Auslandes. Central-Atlas für Zoologische Gärten, Dresden.

Ronquist, F. and Huelsenbeck, J. P. 2003. MrBayes 3: Bayesian phylogenetic inference under mixed models. Bioinformatics 19:1572-1574.

Roos, C. 2003. Molecular phylogeny and systematics of Vietnamese leaf monkeys. Pp. 19-23 in T. Nadler, F. Momberg, N. X. Dang, and N. Lormee, eds. Vietnam Primate Conservation Status Review 2002. Fauna \& Flora International and Frankfurt Zoological Society, Hanoi.

Roos, C. 2004. Molecular Evolution and Systematics of Vietnamese leaf monkeys. Pp. 23-28 in T. Nadler, U. Streicher, and H. T. Long, eds. Conservation of Primates in Vietnam. Haki Publishing, Hanoi.

Roos, C. and Geissmann, T. 2001. Molecular phylogeny of the major hylobatid divisions. Mol. Phylogenet. Evol. 19:486-494.

Roos, C., Nadler, T. and Walter, L. 2008. Mitochondrial phylogeny, taxonomy and biogeography of the silvered langur species group (Trachypithecus cristatus). Mol. Phylogenet. Evol. 47:629-636.

Roos, C., Schmitz, J. and Zischler, H. 2004. Primate jumping genes elucidate strepsirrhine phylogeny. Proc. Natl. Acad. Sci. U S A 101:10650-10654.

Roos, C., Thanh, V. N., Walter, L. and Nadler, T. 2007. Molecular systematics of Indochinese Primates. Vietn. J. Primatol. 1:41-53.

Rosenberger, A. L. 1977. Xenothrix and ceboid phylogeny. J. Hum. Evol. 6:461-481.

Rosenberger, A. L. 1984. Fossil New World monkeys dispute the molecular clock. J. Hum. Evol. 13:737-742.

Rosenberger, A. L. 1981. Systematics: The higher taxa. Pp. 9-27 in A. F. Coimbra-Filho, and R. A. Mittermeier, eds. Ecology and Behaviour of Neotropical Primates. Academia Brasileira de Ciencias, Rio de Janeiro.

Rosenblum, L. L., Supriatna, J., Hasan, M. N. and Melnick, D. J. 1997. High mitochondrial DNA diversity with little structure within and among leaf monkey populations (Trachypithecus cristatus and Trachypithecus auratus). Int. J. Primatol. 18:1005-1028.

Rowe, N. 1996. The Pictorial Guide to the Living Primates. Pogonias Press, East Hampton, New York. 
Rumpler, Y., Warter, S., Hauwy, M., Fausser, J. L., Roos, C. and Zinner, D. in press.Comparing chromosomal and mitochondrial phylogenies of sportive lemurs (Genus Lepilemur, Primates). Chromosome Res.

Ryan, S. C. and Dugaiczyk, A. 1989. Newly arisen DNA repeats in primate phylogeny. Proc. Natl. Acad. Sci. U S A 86:9360-9364.

Ryder, O. A. 1986. Species conservation and systematics: the dilemma of subspecies. Trends Ecol. Evol. 1:9-10.

Saitou, N. and Nei, M. 1987. The neighbor-joining method: a new method for reconstructing phylogenetic trees. Mol. Biol. Evol. 4:406-425.

Salem, A. H., Ray, D. A., Xing, J., Callinan, P. A., Myers, J. S., Hedges, D. J., Garber, R. K., Witherspoon, D. J., Jorde, L. B. and Batzer, M. A. 2003. Alu elements and hominid phylogenetics. Proc. Natl. Acad. Sci. U S A 100:12787-12791.

Schmid, C. and Maraia, R. 1992. Transcriptional regulation and transpositional selection of active SINE sequences. Curr. Opin. Genet. Dev. 2:874-882.

Schmitz, J., Ohme, M., Suryobroto, B. and Zischler, H. 2002a. The colugo (Cynocephalus variegatus, Dermoptera): the primates' gliding sister? Mol. Biol. Evol. 19:2308-2312.

Schmitz, J., Ohme, M. and Zischler, H. 2001. SINE insertions in cladistic analyses and the phylogenetic affiliations of Tarsius bancanus to other primates. Genetics 157:777-784.

Schmitz, J., Ohme, M. and Zischler, H. 2002b. The complete mitochondrial sequence of Tarsius bancanus: evidence for an extensive nucleotide compositional plasticity of primate mitochondrial DNA. Mol. Biol. Evol. 19:544-553.

Schmitz, J., Roos, C. and Zischler, H. 2005. Primate phylogeny: molecular evidence from retroposons. Cytogenet. Genome Res. 108:26-37.

Schmitz, J. and Zischler, H. 2003. A novel family of tRNA-derived SINEs in the colugo and two new retrotransposable markers separating dermopterans from primates. Mol. Phylogenet. Evol. 28:341-349.

Schneider, H., Canavez, F. C., Sampaio, I., Moreira, M. A., Tagliaro, C. H. and Seuanez, H. N. 2001. Can molecular data place each neotropical monkey in its own branch? Chromosoma 109:515-523.

Schneider, H., Sampaio, I., Harada, M. L., Barroso, C. M., Schneider, M. P., Czelusniak, J. and Goodman, M. 1996. Molecular phylogeny of the New World monkeys (Platyrrhini, primates) based on two unlinked nuclear genes: IRBP intron 1 and epsilon-globin sequences. Am. J. Phys. Anthropol. 100:153-179.

Schneider, H., Schneider, M. P., Sampaio, I., Harada, M. L., Stanhope, M., Czelusniak, J. and Goodman, M. 1993. Molecular phylogeny of the New World monkeys (Platyrrhini, primates). Mol. Phylogenet. Evol. 2:225-242.

Schrago, C. G. 2007. On the time scale of New World primate diversification. Am. J. Phys. Anthropol. 132:344354.

Schreiber, A., Wang, M. and Kaumanns, W. 1998. Captive Breeding of Squirrel Monkeys, Saimiri sciureus and Saimiri boliviensis: The Problem of Hybrid Groups. Zoo Biology 17:95-109.

Seehausen, O. 2004. Hybridization and adaptive radiation. Trends Ecol. Evol. 19:198-207.

Sen, S. K., Han, K., Wang, J., Lee, J., Wang, H., Callinan, P. A., Dyer, M., Cordaux, R., Liang, P. and Batzer, M. A. 2006. Human genomic deletions mediated by recombination between Alu elements. Am. J. Hum. Genet. 79:41-53.

Shedlock, A. M. and Okada, N. 2000. SINE insertions: powerful tools for molecular systematics. Bioessays 22:148160.

Shedlock, A. M., Takahashi, K. and Okada, N. 2004. SINEs of speciation: tracking lineages with retroposons. Trends Ecol. Evol. 19:545-553.

Shimamura, M., Yasue, H., Ohshima, K., Abe, H., Kato, H., Kishiro, T., Goto, M., Munechika, I. and Okada, N. 1997. Molecular evidence from retroposons that whales form a clade within even-toed ungulates. Nature 388:666-670.

Shimodaira, H. and Hasegawa, M. 1999. Multiple Comparisons of Log-Likelihoods with Applications to Phylogenetic Inference. Mol. Biol. Evol. 16:1114-1116. 
Shoshani, J., Groves, C. P., Simons, E. L. and Gunnell, G. F. 1996. Primate phylogeny: morphological vs. molecular results. Mol. Phylogenet. Evol. 5:102-154.

Silva, B. T. F., Sampaio, M. I. C., Schneider, H., Schneider, M. P. C., Montoya, E., Encarnacion, F., CallegariJacques, S. M. and Salzano, F. M. 1993. Protein electrophoretic variability in Saimiri and the question of its species status. Am. J. Primatol. 29:183-193.

Silva, B. T. F., Sampaio, M. I. C., Schneider, H., Schneider, M. P. C., Montoya, E., Encarnacion, F. and Salzano, F. M. 1992. Natural hybridization between Saimiri taxa in the Peruvian Amazonia. Primates 33:107-113.

Singer, S. S., Schmitz, J., Schwiegk, C. and Zischler, H. 2003. Molecular cladistic markers in New World monkey phylogeny (Platyrrhini, Primates). Mol. Phylogenet. Evol. 26:490-501.

Sinha, A., Datta, A., Madhusudan, M. D. and Mishra, C. 2005. Macaca munzala: a new species from western Arunachal Pradesh, northeastern India. Int. J. Primatol. 26:977-989.

Smit, A. F. and Riggs, A. D. 1995. MIRs are classic, tRNA-derived SINEs that amplified before the mammalian radiation. Nucleic Acids Res. 23:98-102.

Steiper, M. E. and Ruvolo, M. 2003. New World monkey phylogeny based on X-linked G6PD DNA sequences. Mol. Phylogenet. Evol. 27:121-130.

Steiper, M. E. and Young, N. M. 2006. Primate molecular divergence dates. Mol. Phylogenet. Evol. 41:384-394.

Sterner, K. N., Raaum, R. L., Zhang, Y. P., Stewart, C. B. and Disotell, T. R. 2006. Mitochondrial data support an odd-nosed colobine clade. Mol. Phylogenet. Evol. 40:1-7.

Stewart, C. B. and Disotell, T. R. 1998. Primate evolution - in and out of Africa. Curr. Biol. 8:R582-588.

Strasser, E. and Delson, E. 1987. Cladistic analysis of cercopithecid relationships. J. Hum. Evol. 16:81-99.

Strimmer, K. and von Haeseler, A. 1996. Quartet Puzzling: A Quartet Maximum-Likelihood Method for Reconstructing Tree Topologies. Mol. Biol. Evol. 13:964-969.

Swofford, D. L. 2002. PAUP*: Phylogenetic Analysis Using Parsimony (and Other Methods) 4.0b10. Sinauer Associates, Sunderland.

Szalay, F. S. and Delson, E. 1979. Evolutionary History of the Primates. Academic Press, New York.

Taberlet, P., Waits, L. P. and Luikart, G. 1999. Noninvasive genetic sampling: look before you leap. Trends Ecol. Evol. 14:323-327.

Takahashi, K., Terai, Y., Nishida, M. and Okada, N. 2001. Phylogenetic relationships and ancient incomplete lineage sorting among cichlid fishes in Lake Tanganyika as revealed by analysis of the insertion of retroposons. Mol. Biol. Evol. 18:2057-2066.

Takahashi, K., Terai, Y., Nishida, M. and Okada, N. 1998. A novel family of short interspersed repetitive elements (SINEs) from cichlids: the patterns of insertion of SINEs at orthologous loci support the proposed monophyly of four major groups of cichlid fishes in Lake Tanganyika. Mol. Biol. Evol. 15:391407.

Tavaré, S., Marshall, C. R., Will, O., Soligo, C. and Martin, R. D. 2002. Using the fossil record to estimate the age of the last common ancestor of extant primates. Nature 416:726-729.

Thalmann, O., Fischer, A., Lankester, F., Paabo, S. and Vigilant, L. 2007. The complex evolutionary history of gorillas: insights from genomic data. Mol. Biol. Evol. 24:146-158.

Thalmann, O., Hebler, J., Poinar, H. N., Paabo, S. and Vigilant, L. 2004. Unreliable mtDNA data due to nuclear insertions: a cautionary tale from analysis of humans and other great apes. Mol. Ecol. 13:321-335.

Thompson, J. D., Higgins, D. G. and Gibson, T. J. 1994. CLUSTAL W: improving the sensitivity of progressive multiple sequence alignment through sequence weighting, position-specific gap penalties and weight matrix choice. Nucleic Acids Res. 22:4673-4680.

Thorington, R. W. 1985. The taxonomy and distribution of squirrel monkeys (Saimiri) in L. A. Rosenblum, and C. L. Coe, eds. Handbook of squirrel monkey research. Plenum Publishing Corp, New York.

Ting, N. 2008. Mitochondrial relationships and divergence dates of the African colobines: evidence of Miocene origins for the living colobus monkeys. J. Hum. Evol. 55:312-325. 
Ting, N., Tosi, A. J., Li, Y., Zhang, Y. P. and Disotell, T. R. 2008. Phylogenetic incongruence between nuclear and mitochondrial markers in the Asian colobines and the evolution of the langurs and leaf monkeys. Mol. Phylogenet. Evol. 46:466-474.

Tosi, A. J., Morales, J. C. and Melnick, D. J. 2000. Comparison of Y chromosome and mtDNA phylogenies leads to unique inferences of macaque evolutionary history. Mol. Phylogenet. Evol. 17:133-144.

Tosi, A. J., Morales, J. C. and Melnick, D. J. 2002. Y-chromosome and mitochondrial markers in Macaca fascicularis indicate introgression with Indochinese M. mulatta and a biogeographic barrier in the Isthmus of Kra. Int. J. Primatol. 23:161-178.

van de Lagemaat, L. N., Gagnier, L., Medstrand, P. and Mager, D. L. 2005. Genomic deletions and precise removal of transposable elements mediated by short identical DNA segments in primates. Genome Res. 15:1243-1249.

VandeBerg, J. L. and Williams-Blangero, S. 1997. Advantages and limitations of nonhuman primates as models in genetic research on complex diseases. J. Med. Primatol. 26:113-119.

VandeBerg, J. L., Williams-Blangero, S., Moore, C. M., Cheng, M. L. and Abee, C. R. 1990. Genetic relationships among three squirrel monkey types: implications for taxonomy, biomedical research, and captive breeding. Am. J. Primatol. 22:101-111.

Vermeer, J. 1996. EEP SURVEY OF Saimiri., Apeldoorn, Apenheul Primate Park.

von Dornum, M. and Ruvolo, M. 1999. Phylogenetic relationships of the New World monkeys (Primates, platyrrhini) based on nuclear G6PD DNA sequences. Mol. Phylogenet. Evol. 11:459-476.

von Pusch, A. 1942. Die Arten der Gattung Cebus. Z. Säugertierk. 16:183-237.

Waddell, P. J., Kishino, H. and Ota, R. 2001. A phylogenetic foundation for comparative mammalian genomics. Genome Inform. 12:141-154.

Walker, J. A., Kilroy, G. E., Xing, J., Shewale, J., Sinha, S. K. and Batzer, M. A. 2003. Human DNA quantitation using Alu element-based polymerase chain reaction. Anal. Biochem. 315:122-128.

Wallace, A. 1858. On the Tendency of Varieties to Depart Indefinitely from the Original Type. Proceedings of the Linnean Society 3:53-62.

Wallace, R. B., Gomez, H., Felton, A. and Felton, A. M. 2006. On a new species of titi monkey, genus Callicebus Thomas (Primates, Pitheciidae), from western Bolivia with preliminary notes on distribution and abundance. Primate Conserv. 20:29-39.

Wang, W., Forstner, M. R. J., Zhang, Y. P., Liu, Z. M., Wei, Y., Huang, H. Q., Hu, H. G., Xie, Y. X., Wu, D. H. and Melnick, D. J. 1997. Phylogeny of Chinese leaf monkeys using mitochondrial ND3-ND4 gene sequences. Int. J. Primatol. 18.

Warter, S., Hauwy, M., Dutrillaux, B. and Rumpler, Y. 2005. Application of molecular cytogenetics for chromosomal evolution of the Lemuriformes (Prosimians). Cytogenet. Genome Res. 108:197-203.

Weitzel, V., Yang, C. M. and Groves, C. P. 1988. A catalogue of primates in the Singapore Zoological Reference Collection. The Raffles Bulletin of Zoology 36:1-166.

Whitfield, L. S., Lovell-Badge, R. and Goodfellow, P. N. 1993. Rapid sequence evolution of the mammalian sexdetermining gene SRY. Nature 364:713-715.

Whittaker, D. J., Ting, N. and Melnick, D. J. 2006. Molecular phylogenetic affinities of the simakobu monkey (Simias concolor). Mol. Phylogenet. Evol. 39:887-892.

Whybrow, P. J. 1984. Geological and faunal evidence for mammal 'migrations' between Asia and Africa during the Miocene. Cour. Forsch. Inst. Senckenberg 69:189-198.

Whybrow, P. J. 1992. Land movements and species dispersal in S. Jones, R. E. Martin, and D. Pilbeam, eds. The Cambridge Encyclopedia of Human Evolution. Cambridge University Press, Cambridge.

Wyner, Y. M., Johnson, S. E., Stumpf, R. M. and Desalle, R. 2002. Genetic assessment of a white-collared x redfronted lemur hybrid zone at Andringitra, Madagascar. Am. J. Primatol. 57:51-66. 
Xing, J., Wang, H., Han, K., Ray, D. A., Huang, C. H., Chemnick, L. G., Stewart, C. B., Disotell, T. R., Ryder, O. A. and Batzer, M. A. 2005. A mobile element based phylogeny of Old World monkeys. Mol. Phylogenet. Evol. 37:872-880.

Xing, J., Wang, H., Zhang, Y., Ray, D. A., Tosi, A. J., Disotell, T. R. and Batzer, M. A. 2007. A mobile elementbased evolutionary history of guenons (tribe Cercopithecini). BMC Biol. 5:5.

Yoder, A. D., Burns, M. M., Zehr, S., Delefosse, T., Veron, G., Goodman, S. M. and Flynn, J. J. 2003. Single origin of Malagasy Carnivora from an African ancestor. Nature 421:734-737.

Yoder, A. D., Cartmill, M., Ruvolo, M., Smith, K. and Vilgalys, R. 1996. Ancient single origin for Malagasy primates. Proc. Natl. Acad. Sci. U S A 93:5122-5126.

Zhang, Y. P. and Ryder, O. A. 1998. Mitochondrial Cytochrome b Gene Sequences of Old World Monkeys: With Special Reference on Evolution of Asian Colobines. Primates 39:39-49.

Zischler, H., Geisert, H., von Haeseler, A. and Pääbo, S. 1995. A nuclear 'fossil' of the mitochondrial D-loop and the origin of modern humans. Nature 378:489-492.

Zwickl, D. J. 2006. Genetic Algorithm Approaches for the Phylogenetic Analysis of Large Biological Sequence Datasets under the Maximum Likelihood Criterion. PhD thesis, The University of Texas, Austin. 


\section{Supplementary}

\section{Chapter 2}

Supplementary Table 2.1: Accession number of screened BAC clones

\begin{tabular}{|c|c|c|c|c|}
\hline $\begin{array}{l}\text { Callithrix } \\
\text { jacchus }\end{array}$ & $\begin{array}{c}\text { Saimiri } \\
\text { boliviensis }\end{array}$ & $\begin{array}{c}\text { Aotus } \\
\text { nancymaae }\end{array}$ & $\begin{array}{c}\text { Ateles } \\
\text { geoffroyi }\end{array}$ & $\begin{array}{c}\text { Callicebus } \\
\text { moloch }\end{array}$ \\
\hline AC145530 & AC146467 & AC151892 & AC186273 & AC144655 \\
\hline AC150217 & AC151884 & AC153313 & AC188244 & AC146285 \\
\hline AC150604 & AC151887 & AC166195 & AC188245 & AC151890 \\
\hline AC150613 & AC153429 & AC168952 & AC188246 & AC186942 \\
\hline AC150814 & AC161093 & AC169135 & AC188247 & AC185388 \\
\hline AC151019 & AC183844 & AC171393 & AC188248 & AC186116 \\
\hline AC151042 & AC187665 & AC188295 & AC188249 & AC187183 \\
\hline AC151378 & AC188233 & AC188338 & AC188250 & AC187439 \\
\hline AC160512 & AC188238 & AC188344 & AC188251 & AC187643 \\
\hline AC166001 & AC188239 & AC188493 & AC188252 & AC187691 \\
\hline AC166519 & AC188240 & AC189157 & AC188253 & AC187954 \\
\hline AC167294 & AC188241 & AC190001 & AC188254 & AC188269 \\
\hline AC168185 & AC188242 & AC190441 & AC188256 & AC188270 \\
\hline AC182520 & AC188243 & AC193070 & AC188257 & AC188271 \\
\hline AC188221 & AC198552 & AC193272 & AC188258 & AC188273 \\
\hline AC188222 & AC199259 & AC195014 & AC188259 & AC188276 \\
\hline AC188223 & AC199260 & AC196902 & AC188260 & AC188359 \\
\hline AC188224 & AC202236 & AC202244 & AC188261 & AC188361 \\
\hline AC188227 & AC207836 & AC203482 & AC199280 & AC188363 \\
\hline \multirow[t]{14}{*}{ AC212546 } & AC208270 & & AC199282 & AC190019 \\
\hline & AC209604 & & AC200010 & AC191895 \\
\hline & & & AC200012 & AC196347 \\
\hline & & & AC200013 & AC196348 \\
\hline & & & AC200397 & AC198832 \\
\hline & & & AC201730 & AC199677 \\
\hline & & & AC202262 & AC199678 \\
\hline & & & AC202263 & AC199679 \\
\hline & & & AC202264 & AC200000 \\
\hline & & & AC203135 & AC200393 \\
\hline & & & AC203518 & AC200395 \\
\hline & & & & AC203508 \\
\hline & & & & AC203731 \\
\hline & & & & AC205235 \\
\hline
\end{tabular}




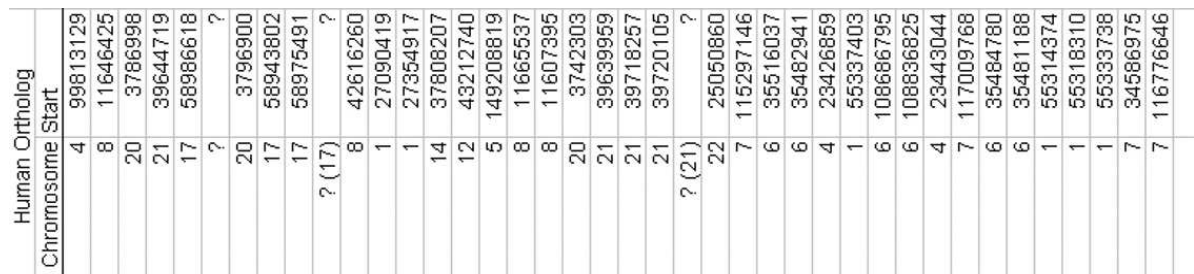

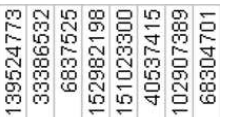
$\sim$ กับ-

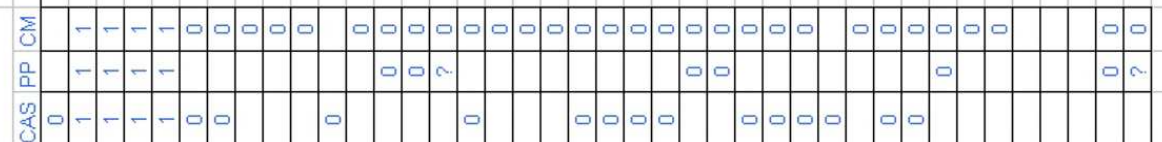

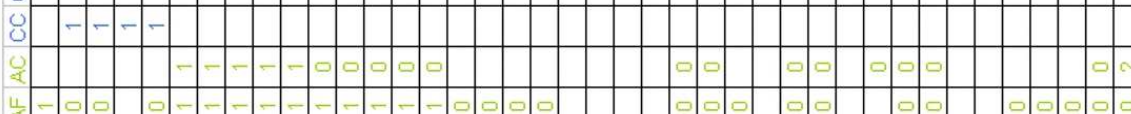

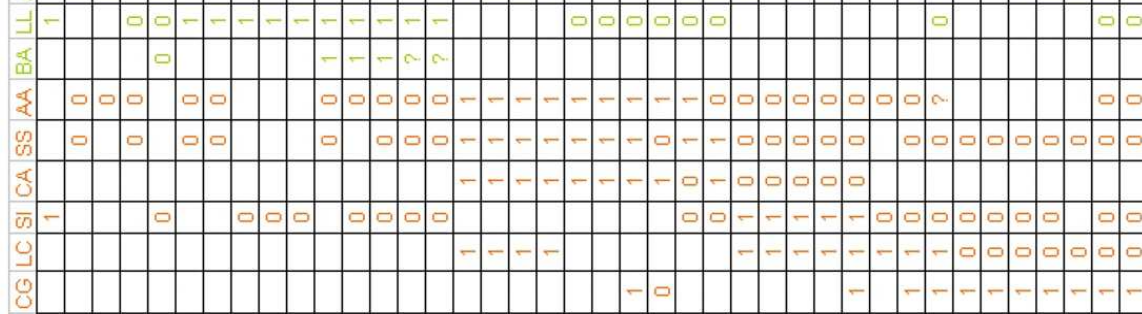

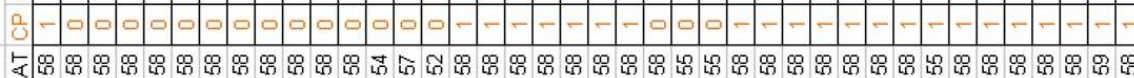
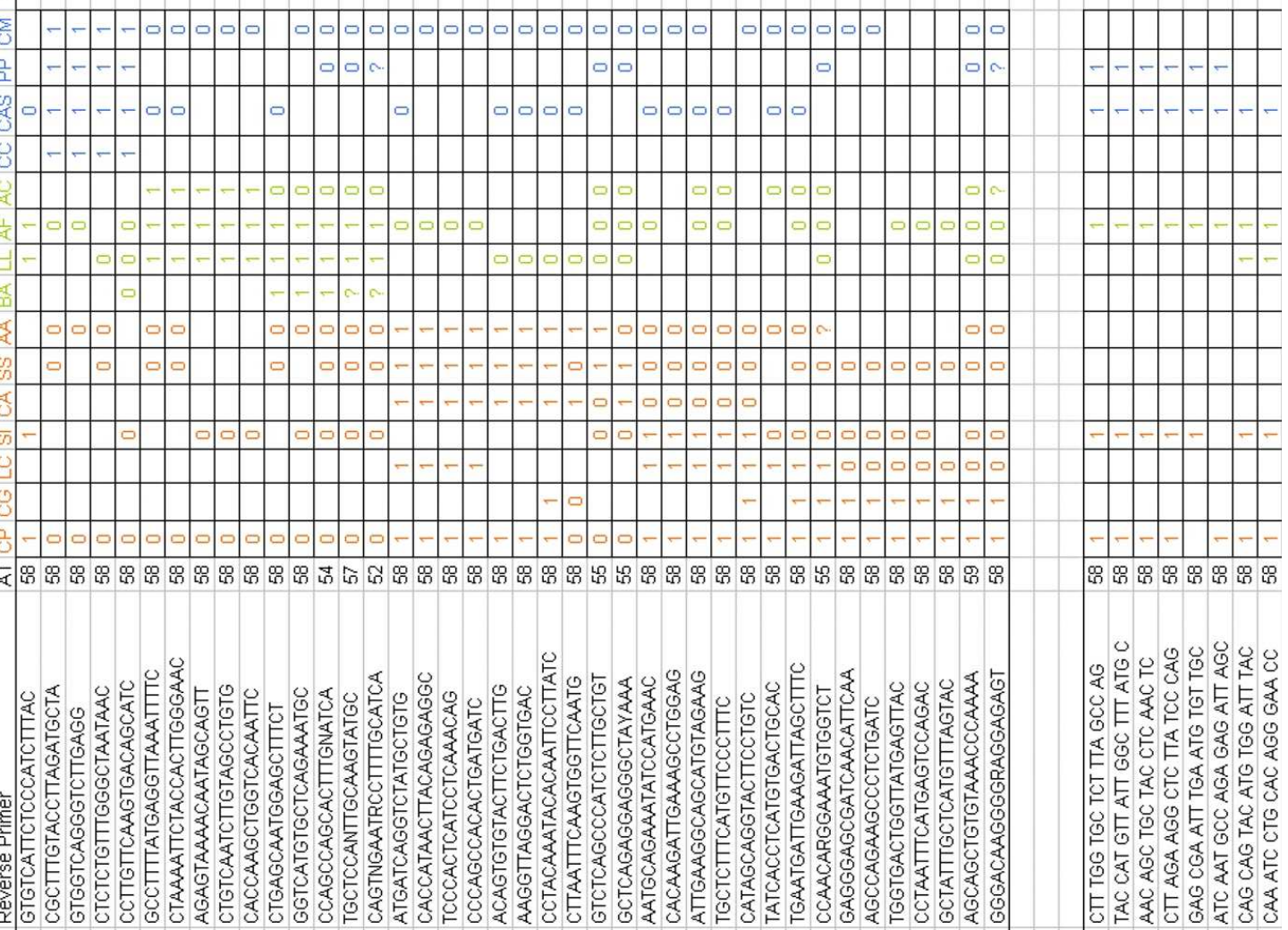

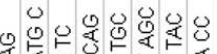

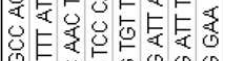

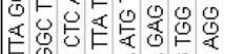

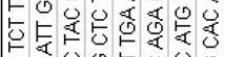

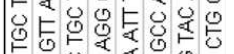

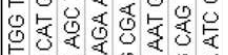

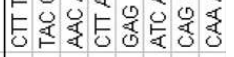

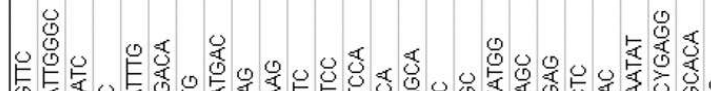

迢造起

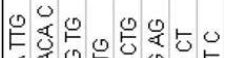

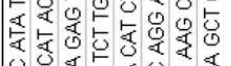

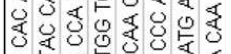

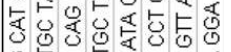

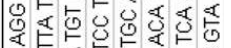

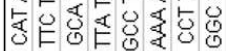

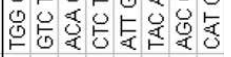

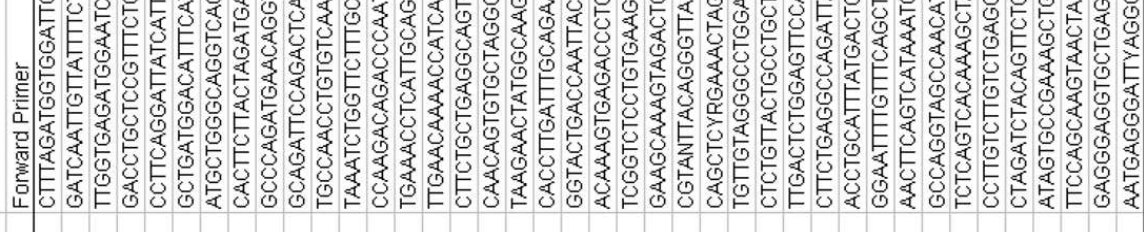

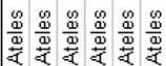

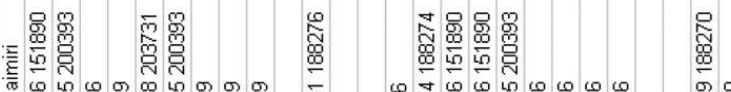

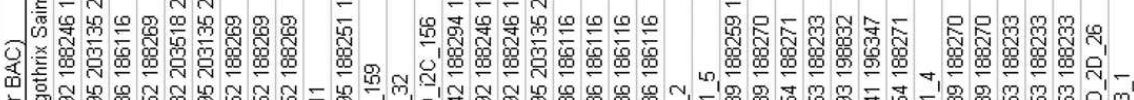

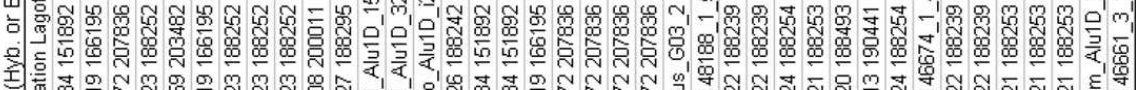

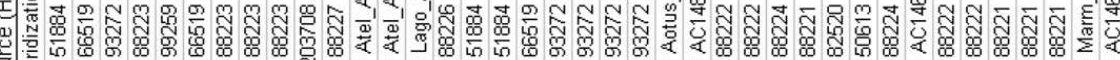

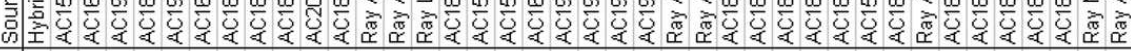

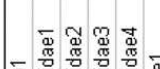

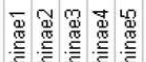

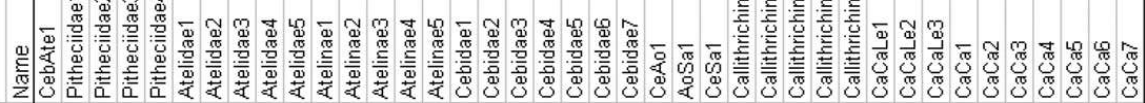
o

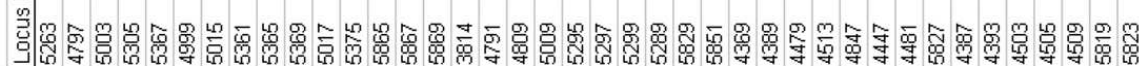
ำ- 


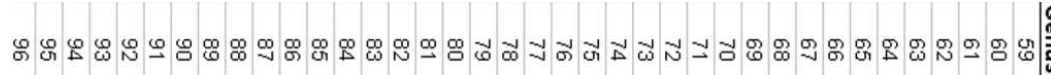
๗

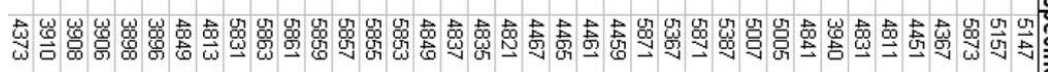
जु

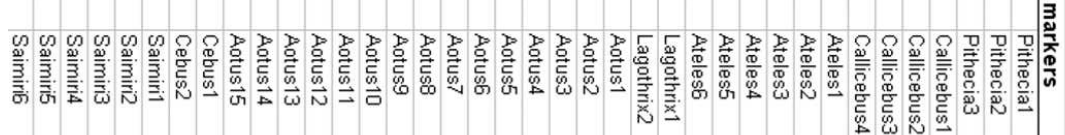

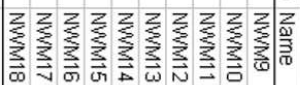

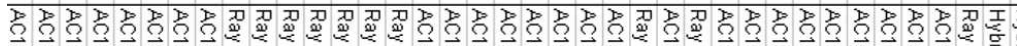

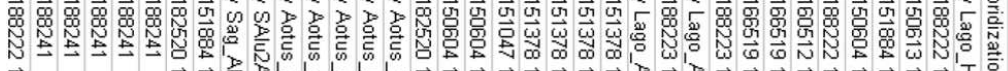

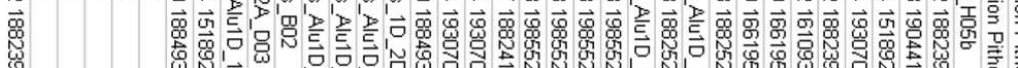
$\overrightarrow{\mathrm{O}}$

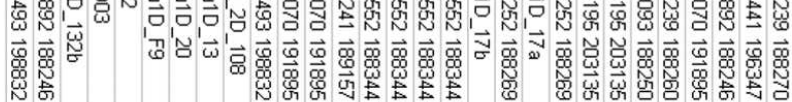

$$
\begin{aligned}
& \overrightarrow{\vec{G}}
\end{aligned}
$$

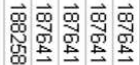

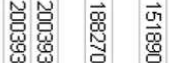

$$
\begin{aligned}
& \text { 認 }
\end{aligned}
$$

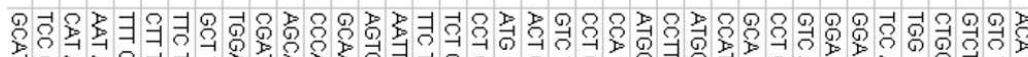

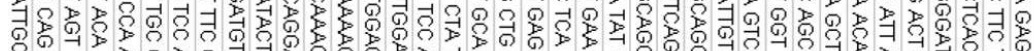

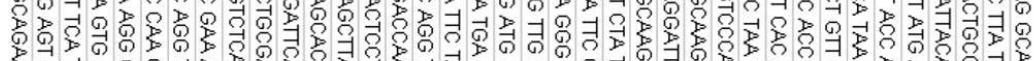

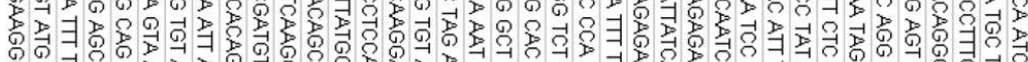

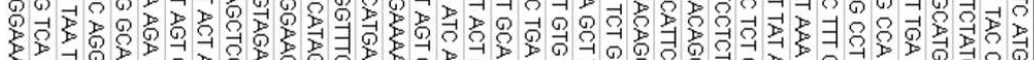
क人

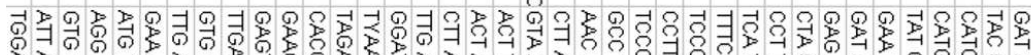

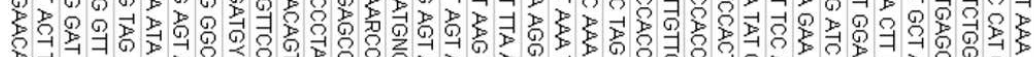

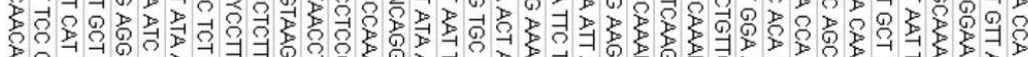

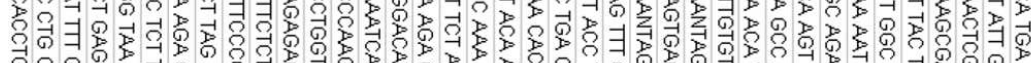

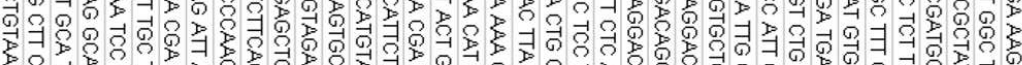

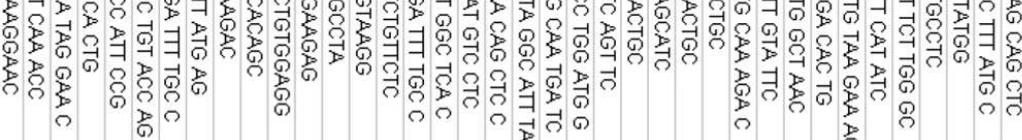

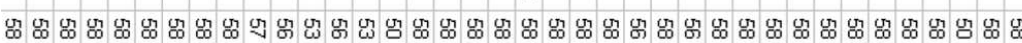

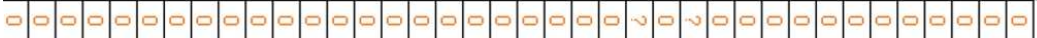

\begin{tabular}{|c|c|c|c|c|c|c|c|c|c|c|c|c|c|c|c|c|c|c|c|c|c|c|}
\hline & & & & & & & & & & & & & & & & & & & & & & \\
\hline & & & & & & & & & & 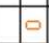 & & ○ & & & & & & & & & & \\
\hline & & & & & & & 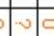 & & & 0 & & & $\sim$ & & 100 & 10 & & & & & & 10 \\
\hline & 0.0 & -1 & $\rightarrow \rightarrow$ & 0 & 0 & 0 & C & & & 0 & 0 & 0 & & & & & & & 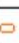 & & & \\
\hline & & & & $\circ$ & & 0 & 0 & & & 0 & 0 & & $\circ$ & 0 & & & 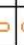 & & & & 0 & \\
\hline & 0 & 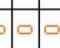 & 00 & $\rightarrow-$ & $\rightarrow$ & & - & & & $\rightarrow-$ & $\rightarrow$ & & $\sim$ & $\sim$ & 0 & 0 & & = & & 5 & 0 & \\
\hline & & & & & & & & & & & & & 0 & 0 & & 0 & & & & & 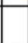 & \\
\hline & & & & 0 & 0 & 0 & 101 & & & & & & $\rightarrow$ & 10 & 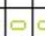 & 80 & & 5 & & & 0 & \\
\hline & 10 & 10 & 0 & 0 & 8 & 0 & 0 & & a & 0 & & & 0 & 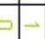 & + & 1 & & & 0 & & 0 & \\
\hline & & & & 0 & & & 0 & & & 0 & 0 & & 0 & 0 & 0 & 0 & & & & 5 & 0 & \\
\hline & & & & & & & & & & & & & 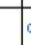 & 0 & & ] & & & & & & 100 \\
\hline & & & & & & & & & & & & & & - & & 0 & & & & & & 10 \\
\hline & & & & & 0 & & $\sim c$ & & & & & & & 0 & & & & & & & & $\rightarrow-$ \\
\hline & & & & & & & & & & & & & & & $c$ & 10 & & & & & $c$ & c \\
\hline
\end{tabular}

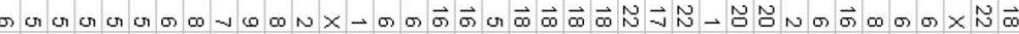

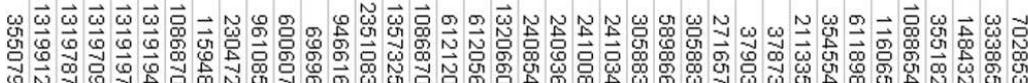

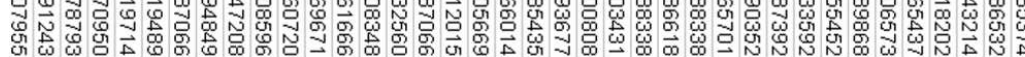

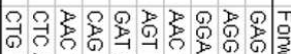
बี

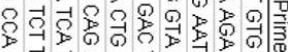

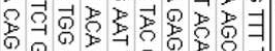

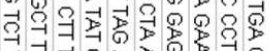

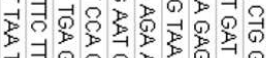
ने

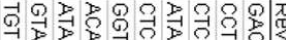

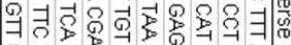

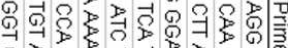
จ ने बै?

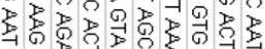

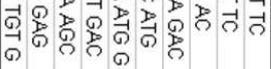

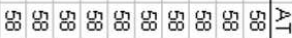

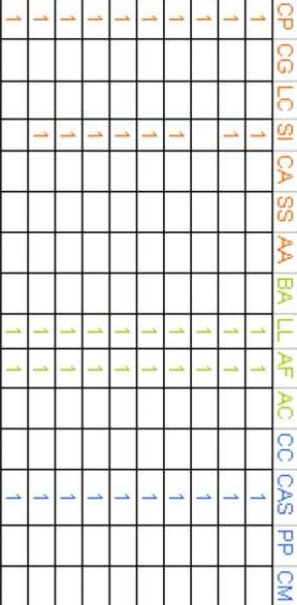
$\times \vec{\omega} \infty \vec{\Delta} \omega \vec{\omega}+\vec{N}$

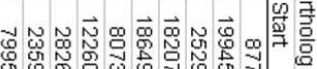
에 


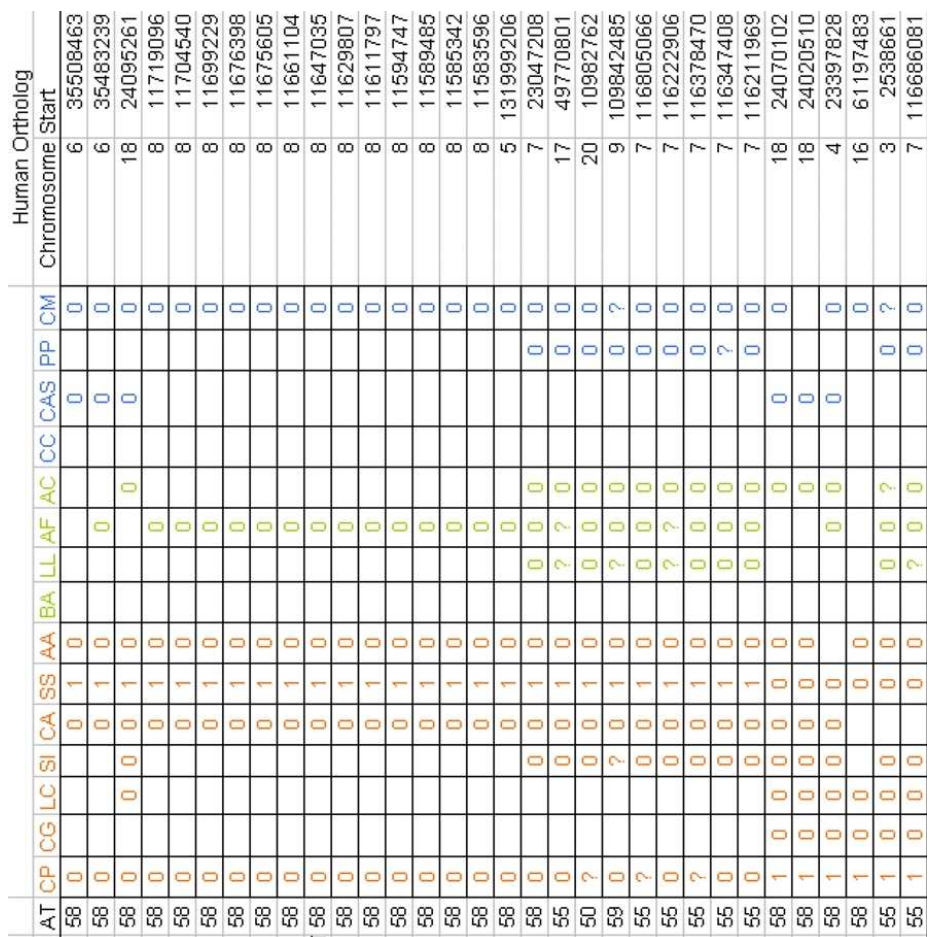

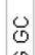
秀。 i.0

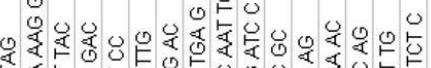

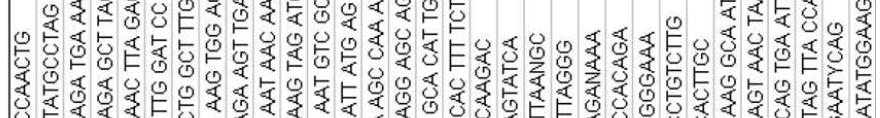

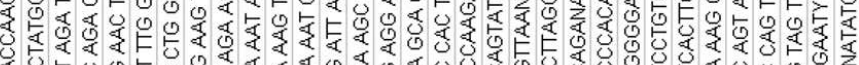

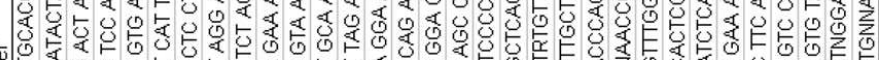

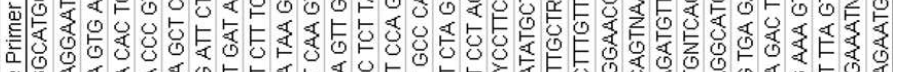

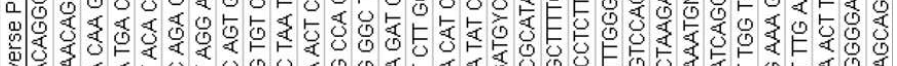

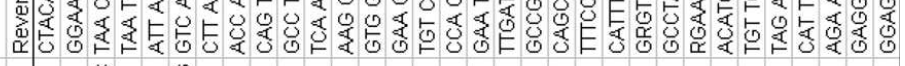

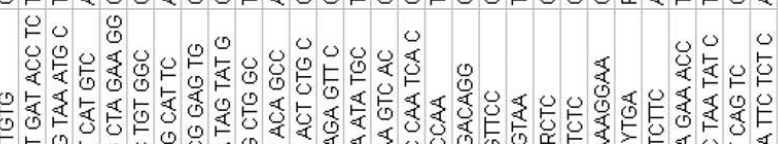

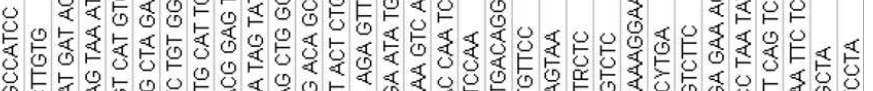

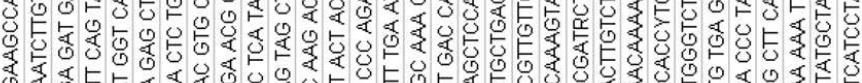

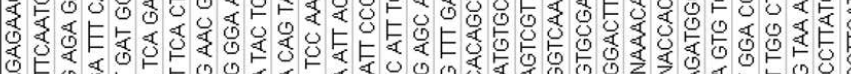

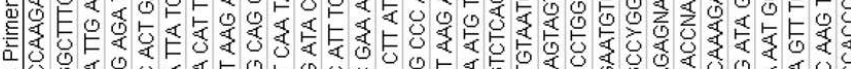
等

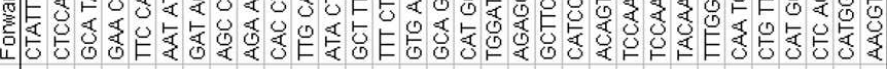

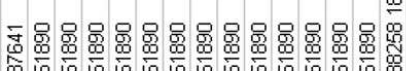

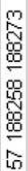

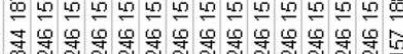

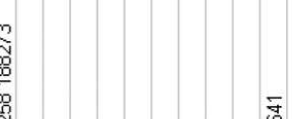

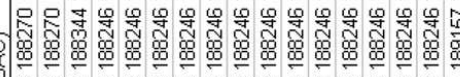

高 声志芯

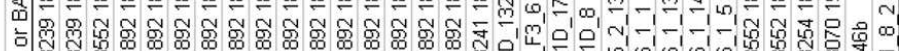

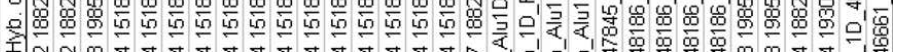

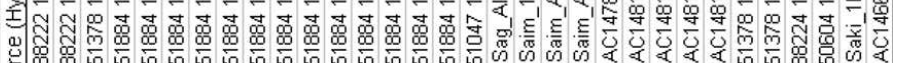

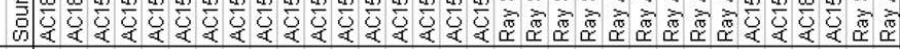

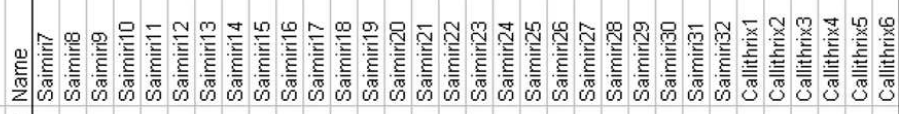

"5

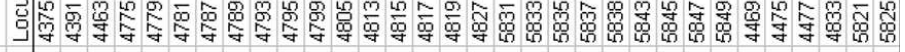

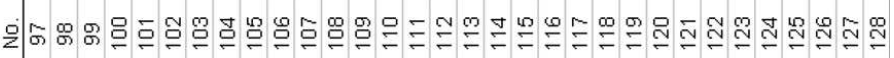

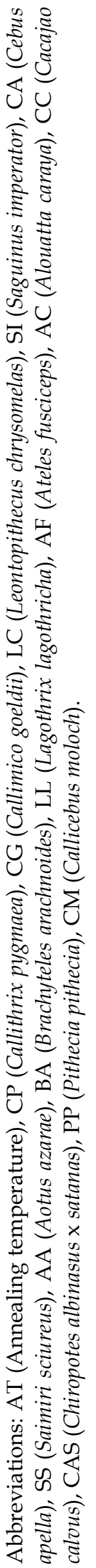




\section{Chapter 5}

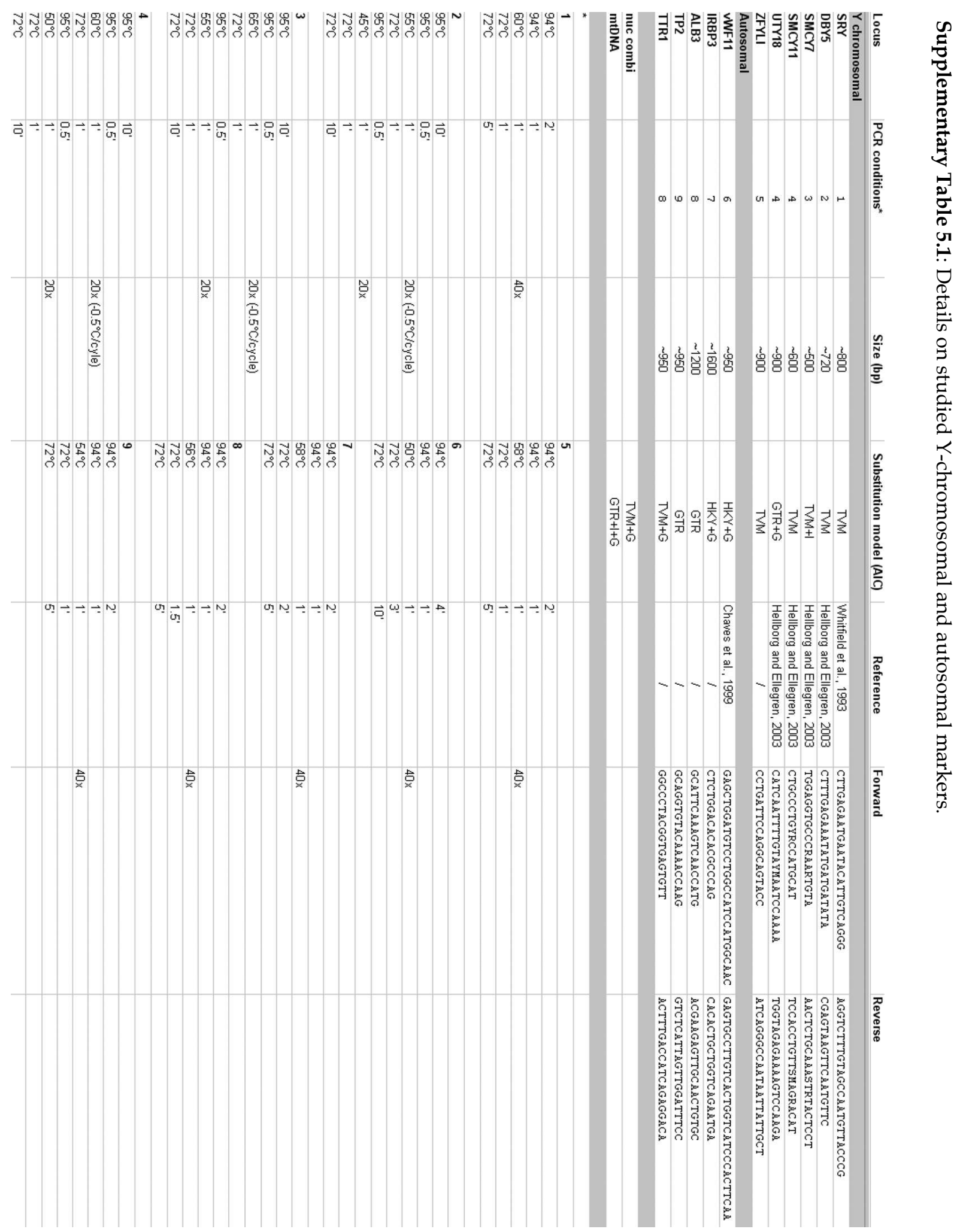




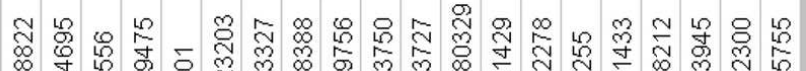

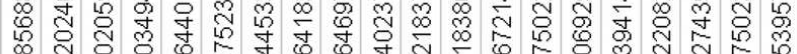
舟

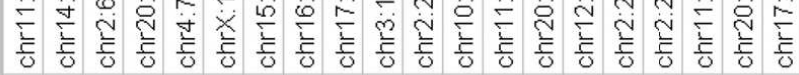

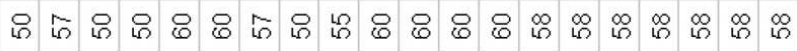

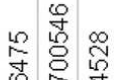

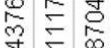
는 串串皿

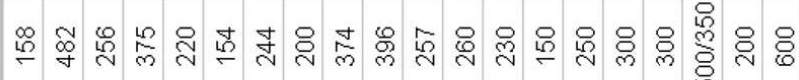

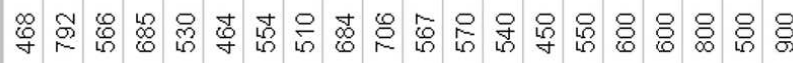

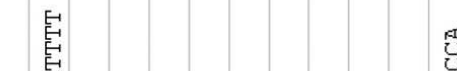

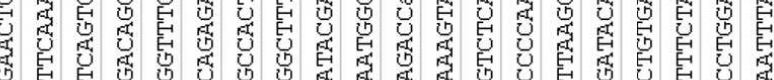

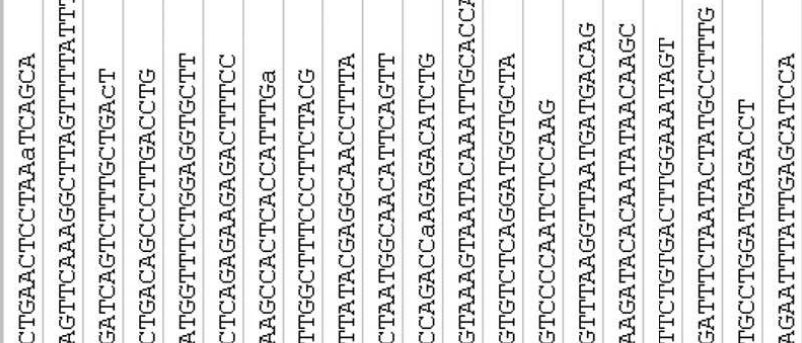

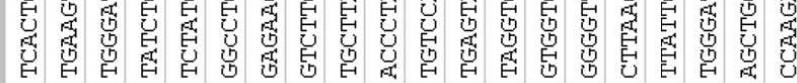
嚁

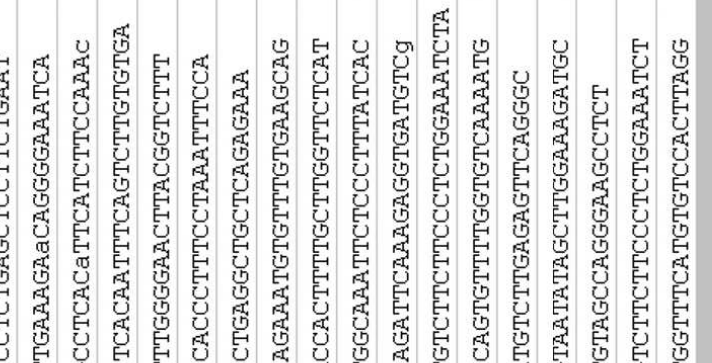

$\stackrel{4}{\perp}$

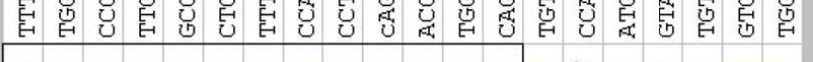

$z_{0}^{z}$

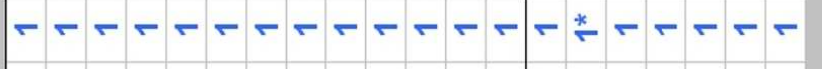

z.

$\sum_{0}$

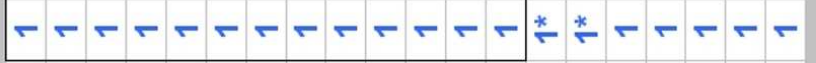

$\frac{a}{u}$

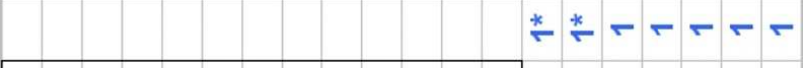

$\stackrel{*}{\sim}+-\cdot-$

은

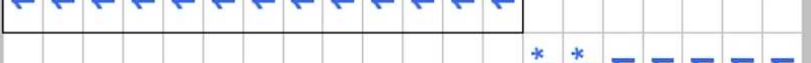

***-r +2

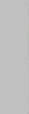

$r-r$

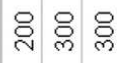

응 : : :

क r-r-r-r+r-

山

魚

u

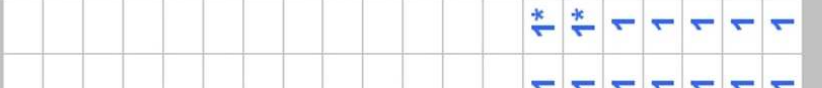

$r+r$

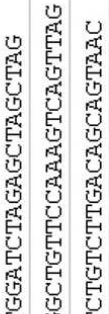

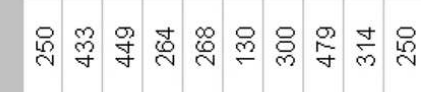

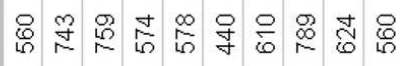

4

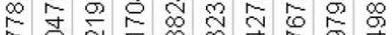

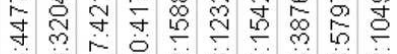

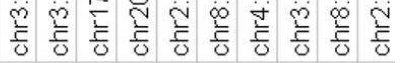

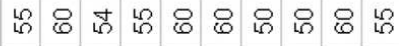




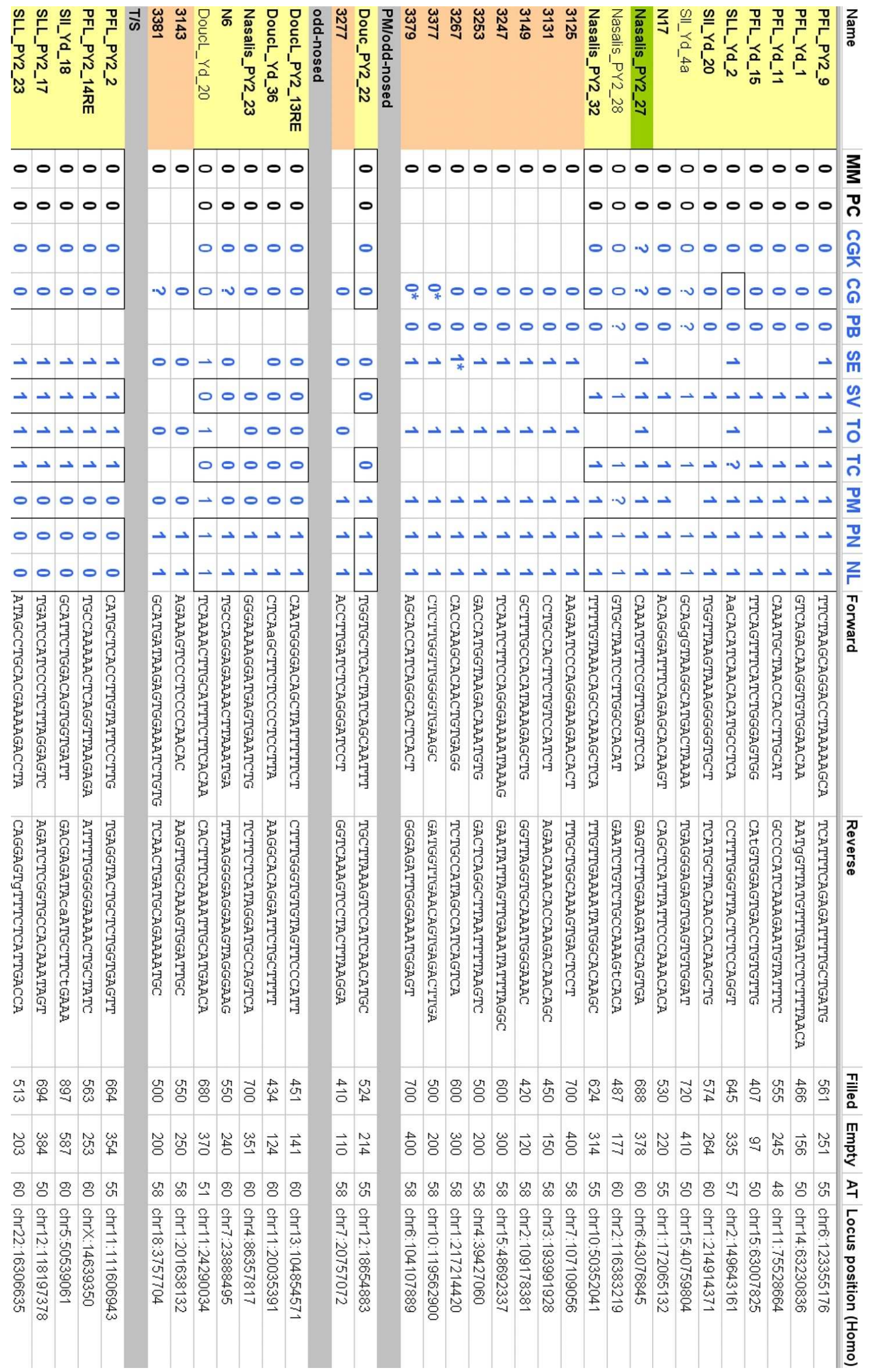




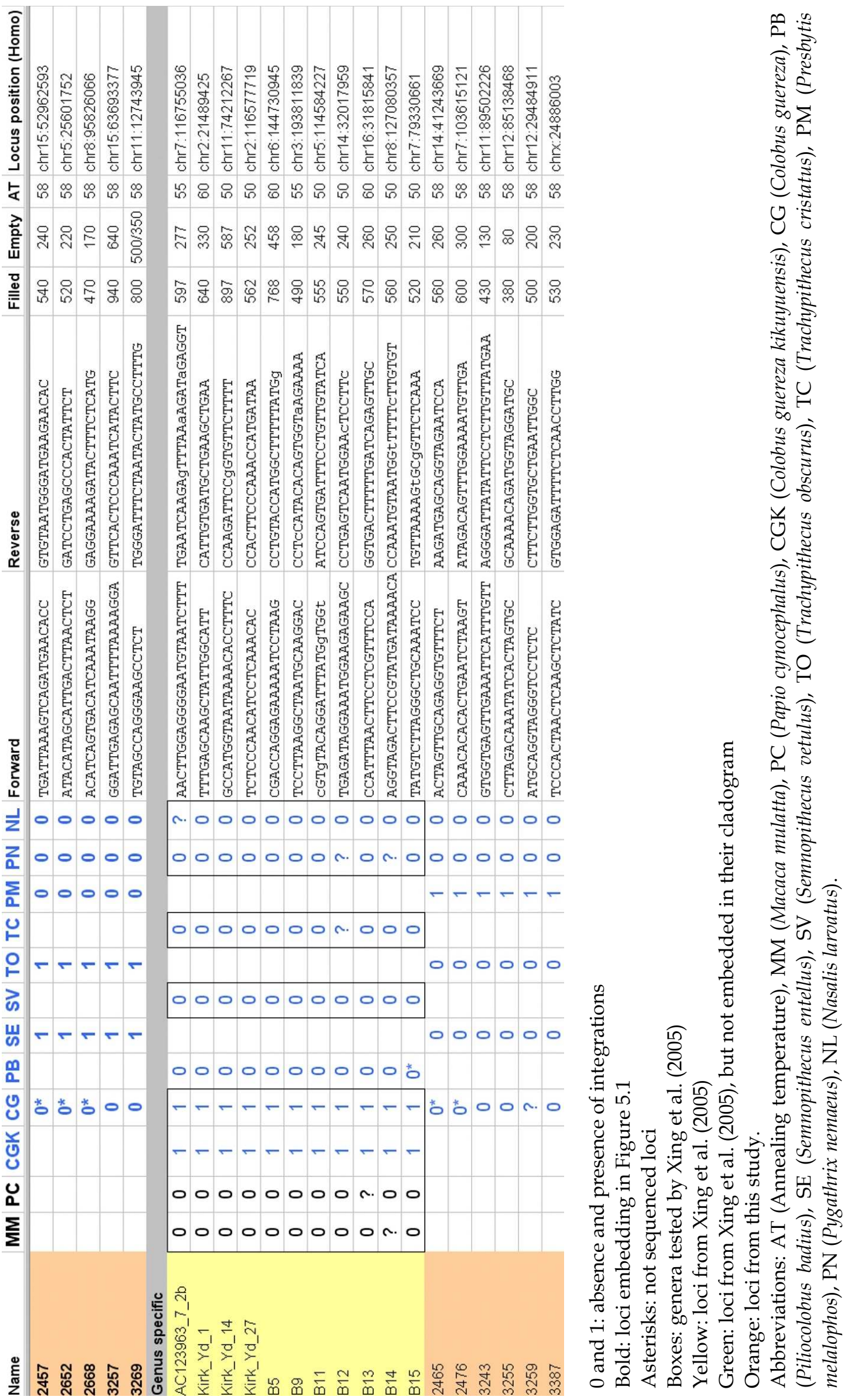


Supplementary Table 5.3: Accession numbers from GenBank of complete mitochondrial genome sequences

\begin{tabular}{ll}
\hline Species & Accession number \\
\hline Homo sapiens & AC000021 \\
Pan troglodytes & EU095335 \\
Papio hamadryas & NC001992 \\
Chlorocebus aethiops & NC007009 \\
Colobus guereza & NC006901 \\
Piliocolobus badius & NC008219 \\
Semnopithecus entellus & NC008215 \\
Trachypithecus obscurus & NC006900 \\
Presbytis melalophos & NC008217 \\
Pygathrix nemaeus & NC008220 \\
Nasalis larvatus & NC008216 \\
\hline
\end{tabular}





\section{Danksagung}

Ich bedanke mich herzlich bei PD Dr. Lutz Walter, der mir die Möglichkeit gegeben hat in seiner Forschergruppe im Rahmen einer Doktorarbeit weiter an diesem interessanten Thema $\mathrm{zu}$ arbeiten.

Ein grosses Dankeschön geht natürlich an Christian Roos mit seinem unfassbaren Schatz an Primatenmaterial. Es ist wirklich spannend mit so wertvollen Proben zu arbeiten, und sei es auch nur der Yeti. Er hatte immer ein offenes Ohr für Probleme oder Anregungen jeglicher Art, ob privat oder beruflich. Die Abende mit Augustiner, Weisswürsten und den Schnecken sind unvergesslich. Ausserdem hat er viel Geduld mit mir bewiesen... aber hey, ich auch mit ihm. Ohne Ihn wäre diese Arbeit nicht zustande gekommen.

Ein weiterer Dank geht selbstverständlich auch an meinen Referenten Prof. Dr. Peter Kappeler und Korreferenten Prof. Dr. Ernst Wimmer.

Ich möchte mich bei allen derzeitigen und ehemaligen Primatengenetikern für das gute Arbeitsklima, für das gemeinsame Speisen in der Mensa und sonstige Freizeit- und Laboraktivitäten bedanken.

Ein riesengroßer Dank geht an Christiane Schwarz, die mir unglaublich viel dabei geholfen hat die Mengen an Laborarbeit zu bewältigen.

Für die schönen Abende ausserhalb des Labors, die natürlich nie etwas mit der Arbeit zu tun hatten, möchte ich mich bei Anna, Alex, Philip, Conny, Wolf und Meike bedanken. Das Gleiche gilt für meine Freunde Andy, Kristof und Lu sowie für die über Deutschland verstreuten Reste.

Schließlich möchte ich mich noch bei meinen Eltern bedanken, die irgendwie ja hauptverantwortlich für mich sind, und bei Laura, die mich ertragen hat, mich aufgebaut hat, mir Ratschläge gegeben hat und immer für mich da war. 


\title{
Lebenslauf
}

\author{
Martin Peter Osterholz \\ Diplom-Biologe \\ geboren am 26.02.1980 in Brunsbüttel \\ Staatsangehörigkeit: deutsch
}

seit 2005

Anfertigung der vorliegenden Dissertation in der Forschergruppe Primatengenetik bei PD Dr. Lutz Walter am Deutschen Primatenzentrum in Göttingen

2005

Abschluss des Studiums zum Diplom-Biologen

$2004-2005$

Anfertigung der Diplomarbeit „Untersuchungen von SINEIntegrationen im Genom der Colobidae,, in der Forschergruppe Primatengenetik bei PD Dr. Lutz Walter am Deutschen Primatenzentrum in Göttingen

$1999-2004$

Studium im Fachbereich Biologie an der Georg-August Universität in Göttingen

1999

Abitur

1990 - 1999

Gymnasium Marne

$1986-1990$

Grundschule Helse 